\title{
Abelian groups are polynomially stable
}

\author{
Oren Becker * Jonathan Mosheiff ${ }^{\dagger}$
}

\begin{abstract}
In recent years, there has been a considerable amount of interest in stability of equations and their corresponding groups. Here, we initiate the systematic study of the quantitative aspect of this theory. We develop a novel method, inspired by the Ornstein-Weiss quasi-tiling technique, to prove that abelian groups are polynomially stable with respect to permutations, under the normalized Hamming metrics on the groups $\operatorname{Sym}(n)$. In particular, this means that there exists $D \geq 1$ such that for $A, B \in \operatorname{Sym}(n)$, if $A B$ is $\delta$-close to $B A$, then $A$ and $B$ are $\epsilon$-close to a commuting pair of permutations, where $\epsilon \leq O\left(\delta^{1 / D}\right)$. We also observe a property-testing reformulation of this result, yielding efficient testers for certain permutation properties.
\end{abstract}

\section{Introduction}

We begin with an informal presentation of a general framework that originated in [9]. Fix the normalized Hamming metric over $\operatorname{Sym}(n)$ as a measure of proximity between permutations. Let $\left(\sigma_{1}, \ldots, \sigma_{s}\right)$ be permutations in $\operatorname{Sym}(n)$. Suppose that $\left(\sigma_{1}, \ldots, \sigma_{s}\right)$ "almost" satisfy a given finite system of equations $E$. Is it necessarily the case that we can "slightly" modify each $\sigma_{i}$ to some $\tau_{i} \in \operatorname{Sym}(n)$, so that $\left(\tau_{1}, \ldots, \tau_{s}\right)$ satisfy $E$ exactly? The answer depends on the system $E$. In the case where $E$ is comprised of the single equation $X Y=Y X$, it was shown in [1] that the answer is positive. Simply put, "almost commuting permutations are close to commuting permutations", where $\sigma_{1}, \sigma_{2}$ are said to "almost commute" if the permutations $\sigma_{1} \sigma_{2}$ and $\sigma_{2} \sigma_{1}$ are close. On the other hand, for $E$ that is the single equation $X Y^{2}=Y^{3} X$ the answer is negative [9]. In the language of [9], " $X Y=Y X$ is stable, whereas $X Y^{2}=Y^{3} X$ is not".

The main novelty of this paper is that we provide the first results in this area which are quantitative and algorithmic. Let us illustrate this for the system $E=\{X Y=Y X\}$. We seek statements of the form "if the distance between $\sigma_{1} \sigma_{2}$ and $\sigma_{2} \sigma_{1}$ is $\delta$, then there are two commuting permutations $\tau_{1}, \tau_{2}$ such that

\footnotetext{
*Department of Mathematics, Hebrew University, Jerusalem, Israel. e-mail: oren.becker@mail.huji.ac.il. Supported by the ERC.

${ }^{\dagger}$ Department of Computer Science, Hebrew University, Jerusalem, Israel. e-mail: yonatanm@cs.huji.ac.il. Supported by the Adams Fellowship Program of the Israel Academy of Sciences and Humanities.
} 


\section{ABELIAN GROUPS ARE POLYNOMIALLY STABLE}

$\tau_{i}$ is at most $f(\delta)$ away from $\sigma_{i}$ for $i=1,2$ ". We stress that $f=f_{E}$, called the stability rate of $E$, depends solely on $\delta$ but not $\sigma_{1}, \sigma_{2}$ or $n$. Previously, it was only shown that $\lim _{\delta \rightarrow 0} f(\delta)=0$, but nothing was said about the rate at which $f$ tends to zero with $\delta$. This is precisely the kind of results that we achieve here. By stating that our results are algorithmic, we mean that they provide an explicit transformation of $\sigma_{1}, \sigma_{2}$ into $\tau_{1}, \tau_{2}$.

More generally, one may consider the set of equations

$$
E_{\text {comm }}^{d}=\left\{X_{i} X_{j}=X_{j} X_{i} \mid 1 \leq i<j \leq d\right\}
$$

and its stability rate function $f_{d}$. The paper [1] shows that $E_{\text {comm }}^{d}$ is stable, or, in terms of the framework of the present paper, that $\lim _{\delta \rightarrow 0} f_{d}(\delta)=0$ for all $d \in \mathbb{N}$. We give a stronger result by proving, in an algorithmic manner, that

$$
f_{d}(\delta) \leq O\left(\delta^{\frac{1}{D}}\right)
$$

where $D=D(d) \geq d$ is an explicitly given constant. We say that " $E_{\text {comm }}^{d}$ is polynomially stable (with degree at most $D$ )". Our proof employs novel elementary methods, and does not rely on previous results concerning stability. Furthermore, we also prove that

$$
f_{d}(\delta) \geq \Omega\left(\delta^{\frac{1}{d}}\right) .
$$

It remains an open problem to close the gap between the bounds on $f_{d}(\delta)$.

Following [9], the basic observation is that the stability of a set $E$ of equations is best studied in terms of the group presented by taking $E$ as relators, and that stability is a group invariant [1]. We show that the stability rate is a group invariant as well, and then use properties of the group $\mathbb{Z}^{d}$ to study the quantitative stability of the equations $E_{\text {comm }}^{d}$. More specifically, our proof of the aforementioned upper bound is based on a tiling procedure, in the spirit of Ornstein-Weiss quasi-tiling for amenable groups [17] (in the setting of group actions which are not necessarily free). The fact that we work with abelian groups (rather than more general amenable groups) enables a highly efficient tiling procedure via an original application of reduction theory of lattices in $\mathbb{Z}^{d}$. Our use of reduction theory is made through a theorem of Lagarias, Lenstra and Schnorr [14] regarding Korkin-Zolotarev bases [15].

Finally, we examine a connection to the topic of property testing in computer science by rephrasing some of the above notions in terms of a certain canonical testing algorithm (this connection, in the non-quantitative setting, is the subject of [3]). In particular, we show that our result yields an efficient algorithm to test whether a given tuple of permutations in $\operatorname{Sym}(n)$ satisfies the equations $E_{\text {comm }}^{d}$.

The rest of the introduction is organized as follows. Section 1.1 paraphrases definitions and results from the theory of stability in permutations. In Section 1.2 , we define the new, more delicate, notion of quantitative stability, and state our main theorems. Section 1.3 explores the connection between stability and property testing, and Section 1.4 discusses previous work. 


\subsection{A review of stability in permutations}

For a set $S$, we write $\mathbb{F}_{S}$ for the free group based on $S$. We also write $S^{-1} \subseteq \mathbb{F}_{S}$ for the set of inverses of elements of $S$ and $S^{ \pm}=S \cup S^{-1}$. Fix a finite set of variables $S=\left\{s_{1}, \ldots, s_{m}\right\}$. An $S$-assignment is a function $\Phi: S \rightarrow \operatorname{Sym}(n)$ for some positive integer $n$, i.e., we assign permutations to the variables, all of which are in $\operatorname{Sym}(n)$ for the same $n$. We naturally extend $\Phi$ to the domain $\mathbb{F}_{S}$ via $\Phi\left(s^{-1}\right)=\Phi(s)^{-1}$ if $s \in S$, and $\Phi\left(w_{1} \cdots w_{t}\right)=\Phi\left(w_{1}\right) \cdots \Phi\left(w_{t}\right)$ if $w_{1}, \ldots, w_{t} \in$ $S^{ \pm}$. In other words, an $S$-assignment $\Phi$ is also regarded as a homomorphism from $\mathbb{F}_{S}$ to a finite symmetric group. Hence, $\Phi$ naturally describes a group action of $\mathbb{F}_{S}$ on the finite set $[n]=\{1, \ldots, n\}$.

An $S$-equation-set (or equation-set over the set $S$ of variables) is a finite ${ }^{1}$ set $E \subseteq \mathbb{F}_{S}$. An assignment $\Phi$ is said to be an $E$-solution if $\Phi(w)$ is the identity permutation $1=1_{\operatorname{Sym}(n)}$ for every $w \in E$. Equivalently, $\Phi$ is an $E$-solution if, when regarded as a homomorphism $\mathbb{F}_{S} \rightarrow \operatorname{Sym}(n)$, it factors through the quotient map $\pi: \mathbb{F}_{S} \rightarrow \mathbb{F}_{S} /\langle\langle E\rangle\rangle$. Namely, if $\Phi=g \circ \pi$ for some homomorphism $g: \mathbb{F}_{S} /\langle\langle E\rangle\rangle \rightarrow \operatorname{Sym}(n)$. Here and throughout, $\langle\langle E\rangle\rangle$ denotes the normal closure of a subset $E$ of a group (the containing group will always be clear from context).

Example 1.1. To relate these definitions to the canonical example of almost commuting pairs of permutation, let $S=\left\{s_{1}, s_{2}\right\}$ and $E=\left\{s_{1} s_{2} s_{1}^{-1} s_{2}^{-1}\right\}$, and consider an $S$-assignment $\Phi$. Note that $\Phi\left(s_{1}\right)$ and $\Phi\left(s_{2}\right)$ commute if and only if $\Phi$ is an $E$-solution. It may be helpful to keep this example in mind when reading up to the end of Section 1.2.

Definition 1.2. For $n \in \mathbb{N}$, the normalized Hamming distance $d_{n}$ on $\operatorname{Sym}(n)$ is defined by $d_{n}\left(\sigma_{1}, \sigma_{2}\right)=\frac{1}{n}\left|\left\{x \in[n] \mid \sigma_{1}(x) \neq \sigma_{2}(x)\right\}\right|$ for $\sigma_{1}, \sigma_{2} \in \operatorname{Sym}(n)$. Also, if $\Phi, \Psi$ are assignments over some finite set of variables $S$, we define $d_{n}(\Phi, \Psi)=\sum_{s \in S} d_{n}(\Phi(s), \Psi(s))$.

Note that $d_{n}$ is bi-invariant, namely, $d_{n}\left(\tau \cdot \sigma_{1} \cdot v, \tau \cdot \sigma_{2} \cdot v\right)=d_{n}\left(\sigma_{1}, \sigma_{2}\right)$ for every $\tau, v \in \operatorname{Sym}(n)$. In particular, $d_{n}\left(\sigma_{1}, \sigma_{2}\right)=d_{n}\left(\sigma_{1} \cdot \sigma_{2}^{-1}, 1\right)$.

Given an $S$-equation-set $E$ and an $S$-assignment $\Phi$, one may ask how close $\Phi$ is to being an $E$-solution. This notion can be interpreted in two different ways. Locally, one can count the points in $[n]=\{1, \ldots, n\}$ on which $\Phi$ violates the equation-set $E$. Taking a global view, one measures how much work, that is, change of permutation entries, is needed to "correct" $\Phi$ into an $E$-solution. We proceed to define the local defect and the global defect of an assignment.

Definition 1.3. Fix a finite set of variables $S$ and an $S$-equation-set $E$, and let $\Phi: S \rightarrow \operatorname{Sym}(n)$ be an $S$-assignment.

(i) The local defect of $\Phi$ with respect to $E$ is

$$
L_{E}(\Phi)=\sum_{w \in E} d_{n}(\Phi(w), 1)=\frac{1}{n} \sum_{w \in E}|\{x \in[n] \mid \Phi(w)(x) \neq x\}| .
$$

\footnotetext{
${ }^{1}$ See [4] for an extension of these definitions to infinite equation-sets.
} 


\section{ABELIAN GROUPS ARE POLYNOMIALLY STABLE}

(ii) The global defect of $\Phi$ with respect to $E$ is

$$
G_{E}(\Phi)=\min \mid\left\{d_{n}(\Phi, \Psi) \mid \Psi: S \rightarrow \operatorname{Sym}(n) \text { is an E-solution }\right\} \mid .
$$

Note that $L_{E}(\Phi)$ and $G_{E}(\Phi)$ are in the ranges $[0,|E|]$ and $[0,|S|]$, respectively. Also, the conditions (i) $\Phi$ is an $E$-solution, (ii) $L_{E}(\Phi)=0$ and (iii) $G_{E}(\Phi)=0$, are all equivalent.

We wish to study the possible divergence between these two types of defect. It is not hard to see (Lemma A.1) that $G_{E}(\Phi) \geq \Omega\left(L_{E}(\Phi)\right.$ ). Indeed, changing a single entry in one permutation $\Phi(s)$, resolves, at best, the violation of $E$ at a constant number of points $x \in[n]$. In this work we seek a reverse inequality, i.e., an upper bound on the global defect in terms of the local defect.

Definition 1.4. Let $E$ be an $S$-equation-set. We define its stability rate $\mathrm{SR}_{E}$ : $(0,|E|] \rightarrow[0, \infty)$ by

$$
\operatorname{SR}_{E}(\delta)=\sup \left\{G_{E}(\Phi) \mid \Phi: S \rightarrow \operatorname{Sym}(n) \text {, where } n \in \mathbb{N} \text { and } L_{E}(\Phi) \leq \delta\right\} .
$$

Note that $\mathrm{SR}_{E}(\delta)$ is a monotone nondecreasing function.

Definition 1.5. [9] If $\lim _{\delta \rightarrow 0} \operatorname{SR}_{E}(\delta)=0$ then $E$ is said to be stable (in permutations).

The theory of equation-sets in permutations is related to group theory via a key observation, given below as Proposition 1.7.

Definition 1.6. Let $E_{1}$ and $E_{2}$ be equation-sets over the respective finite sets of variables $S_{1}$ and $S_{2}$. If the groups $\mathbb{F}_{S_{1}} /\left\langle\left\langle E_{1}\right\rangle\right\rangle$ and $\mathbb{F}_{S_{2}} /\left\langle\left\langle E_{2}\right\rangle\right\rangle$ are isomorphic then $E_{1}$ and $E_{2}$ are called equivalent.

Proposition 1.7. [1] Let $E_{1}$ and $E_{2}$ be equivalent equation-sets. Then $E_{1}$ is stable if and only if $E_{2}$ is stable.

Proposition 1.7 allows us to regard stability as a group invariant:

Definition 1.8. [1] Let $\Gamma$ be a finitely presented group. That is, $\Gamma \cong \mathbb{F}_{S} /\langle\langle E\rangle\rangle$ for some finite sets $S$ and $E \subseteq \mathbb{F}_{S}$. Then, $\Gamma$ is called stable if $E$ is a stable equation-set.

This definition enables us to apply the properties of a group in order to study the stability of its defining set of equations. See Section 1.4 for previous results obtained by this method. The following result is of particular interest to us in the context of this work.

Theorem 1.9. [1] Every finitely generated abelian group is stable.

The proof of Theorem 1.9 in [1] is not algorithmic. That is, it does not describe an explicit transformation that maps an assignment with small local defect to a nearby $E$-solution. With some work, it is possible in principle to extract such an algorithm from the idea presented in [1], by looking further into the proof a theorem of Elek and Szabo [7] used there. However, such an approach would perform poorly in the quantitative sense described below. 


\subsection{Quantitative stability}

We turn to discuss the main new definitions introduced in this work, which deals with quantitative stability. The basis for quantitative stability is a stronger version of Proposition 1.7, given in Proposition 1.11 below. Namely, we show that stability can be refined, yet remain a group invariant, by considering the rate at which $\mathrm{SR}_{E}$ converges to 0 as $\delta \rightarrow 0$. We now make this claim precise.

Definition 1.10. Let $F_{1}, F_{2}:(0,|E|] \rightarrow[0, \infty)$ be monotone nondecreasing functions. Write $F_{1} \sim F_{2}$ if $F_{1}(\delta) \leq F_{2}(C \delta)+C \delta$ and $F_{2}(\delta) \leq F_{1}(C \delta)+C \delta$ for some $C>0$. Let $\left[F_{1}\right]$ denote the class of $F_{1}$ with regard to this equivalence relation.

The reason for the introduction of the $C \delta$ summand in the above definition is elaborated upon in Remark 2.4.

Proposition 1.11. For every equivalent pair of equation-sets $E_{1}$ and $E_{2}$ we have $\mathrm{SR}_{E_{1}} \sim \mathrm{SR}_{E_{2}}$.

We prove Proposition 1.11 in Section 2. This proposition allows us to define the stability rate of a group through the stability rate of a corresponding equation-set (Definition 1.4).

Definition 1.12. Let $E$ be an $S$-equation-set. The stability rate $\mathrm{SR}_{\Gamma}$ of the group $\Gamma=\mathbb{F}_{S} /\langle\langle E\rangle\rangle$ is the equivalence class $\left[\mathrm{SR}_{E}\right]$.

Our goal in this paper is to show that for an abelian group $\Gamma$, not only does the stability rate converge to 0 as $\delta \rightarrow 0$, but this convergence is fast. This claim can be made precise as follows.

Definition 1.13. Let $F:(0,|E|] \rightarrow[0, \infty)$. We define the degree of $F$ by

$$
\operatorname{deg}(F)=\inf \left\{k \geq 1 \mid F(\delta) \leq \underset{\delta \rightarrow 0}{O}\left(\delta^{\frac{1}{k}}\right)\right\} .
$$

It is possible that $\operatorname{deg}(F)=\infty$. Also, let $\operatorname{deg}([F])=\operatorname{deg}(F)$.

Remark 1.14. Note that $\operatorname{deg}([F])$ is well-defined. Indeed, if $F_{1} \sim F_{2}$ and $F_{1}(\delta) \leq$ $\underset{\delta \rightarrow 0}{O}\left(\delta^{\frac{1}{m}}\right)$ for $m \geq 1$, then $F_{2}(\delta) \leq F_{1}(C \delta)+C \delta \leq \underset{\delta \rightarrow 0}{O}\left(\delta^{\frac{1}{m}}\right)$ as well.

Definition 1.15. In the notation of Definition 1.12, the degree of polynomial stability of both $E$ and $\Gamma$ is defined to be $D=\operatorname{deg}\left(\left[\mathrm{SR}_{E}\right]\right)=\operatorname{deg}\left(\mathrm{SR}_{\Gamma}\right)$. If $D<\infty$, we say that $E$ and $\Gamma$ are polynomially stable.

We can now state our main theorem.

Theorem 1.16. Every finitely generated abelian group is polynomially stable.

Notably, Theorem 1.16 applies to commutator equation-sets, namely equationsets of the form

$$
E_{\mathrm{comm}}^{d}=\left\{s_{i} s_{j} s_{i}^{-1} s_{j}^{-1} \mid 1 \leq i<j \leq d\right\}
$$




\section{ABELIAN GROUPS ARE POLYNOMIALLY STABLE}

over the variables $\left\{s_{1}, \ldots, s_{d}\right\}$ where $d \geq 0$. Indeed, note that $\mathbb{F}_{d} /\left\langle\left\langle E_{\text {comm }}^{d}\right\rangle\right\rangle \cong$ $\mathbb{Z}^{d}$, so $E_{\text {comm }}^{d}$ is polynomially stable. In particular, $d=2$ yields the example of commuting pairs of permutations from the beginning of the introduction.

We complement Theorem 1.16 with a lower bound on the degree of polynomial stability.

Theorem 1.17. For all $d \in \mathbb{N}$, the group $\mathbb{Z}^{d}$ has degree of polynomial stability at least $d$.

We note that, having shown that an abelian group $\Gamma$ is polynomially stable, we are left with the more delicate question of its degree of polynomial stability. Restricting attention to a free abelian group $\mathbb{Z}^{d}$, the proof of Theorem 1.16 yields an upper bound on this degree that grows exponentially in $d$ (see Equation (4.2)). A remaining open problem is to close the large gap between this upper bound, and the lower bound of Theorem 1.17.

We diverge from the practice of providing an outline of the proofs in the introduction since such an outline requires a geometric formulation of stability, which is developed in Section 3. Theorem 1.16 is proved in Section 4, and its proof is outlined in Section 4.1. A reader who is interested in the quickest route to understanding this outline may skip Section 2. Theorem 1.17 is the subject of Section 5 .

\subsection{Application to property testing}

The notions of stability and quantitative stability have a natural interpretation in terms of property testing (For more on this connection see [3]. For background on property testing see $[10,18])$.

Definition 1.18. Fix a nonempty equation-set $E$ over the finite set of variables $S$. A tester for $E$ is an algorithm which takes an assignment $\Phi: S \rightarrow \operatorname{Sym}(n)$ as input, queries the permutations $\{\Phi(s)\}_{s \in S}$ at a constant (in particular, independent of $n$ ) number of entries among $1, \ldots, n$, and decides whether $\Phi$ is an $E$-solution. The algorithm must satisfy the following:

(I) If $\Phi$ is an $E$-solution, the algorithm always accepts.

(II) For some fixed function $\delta:(0, \infty) \times \mathbb{N} \rightarrow(0,1]$, if $\Phi$ is not an $E$-solution, then the algorithm rejects with probability at least $\delta\left(G_{E}(\Phi), n\right)$. This function $\delta$ is called the detection probability of the tester.

The precise term in the literature for this notion is an "adaptive proximityoblivious tester with one-sided error and constant query complexity" (see, [10] Definition 1.7).

The canonical tester $\mathcal{N}_{E}$ for $E$ samples $x \in[n]$ and $w \in E$ uniformly at random from their respective sets. It accepts if $\Phi(w)(x)=x$ and rejects otherwise. Clearly, if $\Phi$ is an $E$-solution then $\mathcal{N}_{E}$ always accepts. If $\Phi$ is not an $E$-solution, then $\mathcal{N}_{E}$ rejects with probability $\frac{1}{|E|} \cdot L_{E}(\Phi)$. Generally, it is desirable for the detection probability function $\delta(\epsilon, n)$ to be uniform, i.e., depend 


\section{OREN BECKER AND JONATHAN MOSHEIFF}

only on $\epsilon=G_{E}(\Phi)$ and not on $n$. In order to show that this is the case for $\mathcal{N}_{E}$, we must consider the function

$$
\begin{aligned}
\delta(\epsilon) & =\inf \left\{\frac{1}{|E|} \cdot L_{E}(\Phi) \mid \Phi \text { is an } S \text {-assignment and } G_{E}(\Phi) \geq \epsilon\right\} \\
& =\inf \left\{\delta \mid \operatorname{SR}_{E}(\delta) \geq \epsilon\right\} .
\end{aligned}
$$

The tester $\mathcal{N}_{E}$ satisfies Condition 1.18(II) if and only if $\delta(\epsilon)$ is positive for every $\epsilon>0$. This is equivalent to the condition that $\lim _{\delta \rightarrow 0} \operatorname{SR}_{E}(\delta)=0$. Hence, $\mathcal{N}_{E}$ admits a uniform detection probability function if and only if $E$ is stable.

Furthermore, the smaller the stability rate of $E$, the larger $\delta$ is. In particular, if $E$ is polynomially stable with degree of polynomial stability $D$, then $\delta(\epsilon)$ is bounded from below by $\Omega\left(\epsilon^{D}\right)$. Therefore, due to Theorem 1.16, the canonical tester $\mathcal{N}_{E}$ has detection probability polynomial in $\epsilon$ and uniform in $n$, whenever $\left.\mathbb{F}_{S} /\langle E\rangle\right\rangle$ is abelian.

In a somewhat weaker formulation of property testing (see [10], Definition 1.6 ), the tester is only required to distinguish between the cases $G_{E}(\Phi)=0$ and $G_{E}(\Phi)>\epsilon$, where $\epsilon>0$ is given as input. In this formulation, the detection probability is required to be larger than some constant, say $\frac{1}{2}$, and one seeks to minimize the number of queries. Note that, for every $E$, the canonical tester $\mathcal{N}_{E}$ can be used to build a tester $\tilde{\mathcal{N}}_{E}$, satisfying this weaker formulation: Given $\epsilon>0$, and an input $\Phi$, the tester $\tilde{\mathcal{N}}_{E}$ runs $\mathcal{N}_{E}$ on $\Phi$ repeatedly for $\log _{1-\delta(\epsilon, n)} \frac{1}{2}=\Theta\left(\frac{1}{\delta(\epsilon, n)}\right)$ independent iterations, and accepts only if $\mathcal{N}_{E}$ accepts in all iterations. Hence, the resulting tester $\tilde{\mathcal{N}}_{E}$ performs $\Theta\left(\frac{1}{\delta(\epsilon, n)}\right)$ queries. In particular, Theorem 1.16 shows that for $E$ such that $\mathbb{F}_{S} /\langle\langle E\rangle$ is abelian, the tester $\tilde{\mathcal{N}}_{E}$ is efficient. Namely, it has constant detection probability, and its number of queries is polynomial in $\frac{1}{\epsilon}$ and does not depend on $n$. No such tester was previously known.

\subsection{Previous work}

The general question of whether almost-solutions are close to solutions, in various contexts, was suggested by Ulam (see [19], Chapter VI). The most studied question of this sort is whether almost-commuting matrices are close to commuting matrices, and the answer depends on the chosen matrix norm and on which kind of matrices is considered (e.g. self-adjoint, unitary, etc.). See the introduction of [1] for a short survey, and $[5,6,11,12]$ for some newer works. In this context, some quantitative results are already known $[8,13]$. The question of (non-quantitative) stability in permutations, under the normalized Hamming metric, was initiated in [9] and developed further in [1]. The former paper proves that finite groups are stable (see our Proposition A.4 for a quantitative version), and the latter proves that abelian groups are stable. Both papers provide examples of non-stable groups as well, and relate stability in permutations to the notion of sofic groups. These results are generalized in [4], which provides a characterization of stability in permutations, among amenable groups, in terms 


\section{ABELIAN GROUPS ARE POLYNOMIALLY STABLE}

of their invariant random subgroups. On the other side of the spectrum (compared to amenable groups), [2] proves that infinite groups with Property (T) are never stable in permutations, and consequently suggests some weaker forms of stability.

\section{The stability rate is a group invariant}

In this section we prove Proposition 1.11. We start with Lemma 2.1, which formalizes the claim that if two $S$-assignments $\Phi$ and $\Psi$ are close to each other, then so are the permutations $\Phi(w)$ and $\Psi(w)$, provided that $w \in \mathbb{F}_{S}$ is a short word. It is helpful to consider $w=w_{1} \cdots w_{t}\left(w_{i} \in S^{ \pm}\right)$as a sequence of directions, namely, $\Phi(w)(x)$ is the endpoint of the path that starts at $x$, moves in the direction $w_{t}$ to $\Phi\left(w_{t}\right)(x)$, continues to $\Phi\left(w_{t-1} w_{t}\right)(x)$, and so on. The immediate observation behind Lemma 2.1, is that as long as this path moves only along "nice" edges, i.e., edges on which $\Phi$ and $\Psi$ agree, it is guaranteed that $\Phi(w)(x)=\Psi(w)(x)$. A similar idea is then used to prove Lemma 2.2 as well.

Lemma 2.1. Let $E$ be an $S$-equation-set, let $\Phi, \Psi: S \rightarrow \operatorname{Sym}(n)$ be $S$-assignments, and let $w=w_{1} \cdots w_{t} \in \mathbb{F}_{S}$ where $w_{i} \in S^{ \pm}$. Then, $d_{n}(\Phi(w), \Psi(w)) \leq t \cdot d_{n}(\Phi, \Psi)$.

Proof. Let $\bar{w}_{i}$ denote the suffix $w_{i} \cdots w_{t}$. By the bi-invariance of $d_{n}$ and the triangle inequality,

$$
\begin{aligned}
d_{n}(\Phi(w), \Psi(w)) & =d_{n}\left((\Psi(w))^{-1} \cdot \Phi(w), 1\right) \\
& \left.\leq \sum_{i=1}^{t} d_{n}\left(\left(\Psi\left(\bar{w}_{i}\right)\right)^{-1} \cdot \Phi\left(\bar{w}_{i}\right), \Psi\left(\bar{w}_{i+1}\right)\right)^{-1} \cdot \Phi\left(\bar{w}_{i+1}\right)\right) \\
& =\sum_{i=1}^{t} d_{n}\left(\left(\Psi\left(w_{i}\right)\right)^{-1} \cdot \Phi\left(w_{i}\right), 1\right)=\sum_{i=1}^{t} d_{n}\left(\Phi\left(w_{i}\right), \Psi\left(w_{i}\right)\right) .
\end{aligned}
$$

Note that each term of the right hand side is at most $d_{n}(\Phi, \Psi)$. Here, if $w_{i}$ is the inverse of a generator, we used the fact that $d_{n}\left(\Phi\left(w_{i}\right), \Psi\left(w_{i}\right)\right)=$ $d_{n}\left(\Phi\left(w_{i}^{-1}\right), \Psi\left(w_{i}^{-1}\right)\right)$. The lemma follows.

Lemma 2.2. Fix an $S$-equation-set $E \subseteq \mathbb{F}_{S}$, and a word $w \in\langle\langle E\rangle\rangle$, written as $w=u_{1} q_{1} u_{1}^{-1} u_{2} q_{2} u_{2}^{-1} \cdots u_{t} q_{t} u_{t}^{-1}$, where $u_{i} \in \mathbb{F}_{S}$ and each $q_{i}$ is an element of $E$ or its inverse. Then, for every $S$-assignment $\Phi: S \rightarrow \operatorname{Sym}(n)$,

$$
d_{n}(\Phi(w), 1) \leq L_{E}(\Phi) \cdot t
$$


Proof. We have

$$
\begin{aligned}
d_{n}(\Phi(w), 1) & =d_{n}\left(\Phi\left(u_{1} q_{1} u_{1}^{-1} u_{2} q_{2} u_{2}^{-1} \cdots u_{t} q_{t} u_{t}^{-1}\right), 1\right) \\
& \leq \sum_{i=1}^{t} d_{n}\left(\Phi\left(u_{i} q_{i} u_{i}^{-1} \cdots u_{t} q_{t} u_{t}^{-1}\right), \Phi\left(u_{i+1} q_{i+1} u_{i+1}^{-1} \cdots u_{t} q_{t} u_{t}^{-1}\right)\right) \\
& =\sum_{i=1}^{t} d_{n}\left(\Phi\left(u_{i} q_{i} u_{i}^{-1}\right), 1\right) \\
& =\sum_{i=1}^{t} d_{n}\left(\Phi\left(q_{i}\right), 1\right) \\
& \leq \sum_{i=1}^{t} \sum_{w \in E} d_{n}(\Phi(w), 1) \\
& =t \cdot L_{E}(\Phi) .
\end{aligned}
$$

The following corollary of Lemma 2.2 will also be useful.

Lemma 2.3. Fix an $S$-equation-set $E$ and a homomorphism $\lambda: \mathbb{F}_{S} \rightarrow \mathbb{F}_{S}$ such that $w$ and $\lambda(w)$ belong to the same coset in $\mathbb{F}_{S} /\langle\langle E\rangle\rangle$ for each $w \in \mathbb{F}_{S}$. Then, there exists a positive $c=c(S, E, \lambda)$ such that for every $S$-assignment $\Phi: S \rightarrow \operatorname{Sym}(n)$, we have $d_{n}(\Phi, \Phi \circ \lambda) \leq c \cdot L_{E}(\Phi)$, where we regard $\Phi \circ \lambda$ as an $S$-assignment by restricting its domain from $\mathbb{F}_{S}$ to $S$.

Proof. Note that

$$
d_{n}(\Phi, \Phi \circ \lambda)=\sum_{s \in S} d_{n}(\Phi(s), \Phi(\lambda(s)))=\sum_{s \in S} d_{n}\left(1, \Phi\left(\lambda(s) \cdot s^{-1}\right) .\right.
$$

Since $\lambda(s) \cdot s^{-1} \in\langle\langle E\rangle$, it follows from Lemma 2.2 that the $s$-term of this sum is at most $O\left(L_{E}(\Phi)\right)$, where the implied constant depends on $s, E$ and $\lambda$. Hence, $d_{n}(\Phi, \Phi \circ \lambda) \leq O\left(L_{E}(\Phi)\right)$.

We turn to prove Proposition 1.11.

Proof of Proposition 1.11. In the course of the proof, when a function whose domain is $\mathbb{F}_{S_{1}}$ or $\mathbb{F}_{S_{2}}$ appears where a function whose domain is $S_{1}$ or $S_{2}$ is expected, the function should be regarded as its respective restriction (for example, when measuring distances between assignments).

Let $E_{1}$ and $E_{2}$ be equivalent equation-sets over the respective finite sets of variables $S_{1}$ and $S_{2}$. By symmetry, it is enough to prove that $\operatorname{SR}_{E_{1}}(\delta) \leq$ $\mathrm{SR}_{E_{2}}(C \delta)+C \delta$ for some $C=C\left(E_{1}, E_{2}\right)$. Equivalently, we need to show that $G_{E_{1}}\left(\Phi_{1}\right) \leq \operatorname{SR}_{E_{2}}(C \delta)+C \delta$ for any given $\delta>0$ and $S_{1}$-assignment $\Phi_{1}: S_{1} \rightarrow$ $\operatorname{Sym}(n)$ with $L_{E_{1}}\left(\Phi_{1}\right) \leq \delta$. 


\section{ABELIAN GROUPS ARE POLYNOMIALLY STABLE}

Our strategy is to "translate" the $S_{1}$-assignment $\Phi_{1}$ into an $S_{2}$-assignment $\Phi_{2}$, find an $E_{2}$-solution $\Psi_{2}$ which is close to $\Phi_{2}$, and pull back $\Psi_{2}$ to an $E_{1}$ solution $\Psi_{1}$. We apply Lemma 2.2 to bound $L_{E_{2}}\left(\Phi_{2}\right)$, and then use Lemmas 2.1 and 2.3 to control the distance between $\Phi_{1}$ and $\Psi_{1}$, yielding an upper bound on $G_{E_{1}}\left(\Phi_{1}\right)$.

We define the machinery needed to map $S_{1}$-assignments to $S_{2}$-assignments and vice versa. Since $E_{1}$ and $E_{2}$ are equivalent equation-sets, there exists a group isomorphism $\theta: \mathbb{F}_{S_{1}} /\left\langle\left\langle E_{1}\right\rangle\right\rangle \rightarrow \mathbb{F}_{S_{2}} /\left\langle\left\langle E_{2}\right\rangle\right\rangle$. Let $\pi_{1}$ denote the quotient map $\mathbb{F}_{S_{1}} \rightarrow \mathbb{F}_{S_{1}} /\left\langle\left\langle E_{1}\right\rangle\right\rangle$, and likewise for $\pi_{2}$. Fix a homomorphism $\lambda_{2}: \mathbb{F}_{S_{2}} \rightarrow \mathbb{F}_{S_{1}}$ such that $\pi_{2}=\theta \circ \pi_{1} \circ \lambda_{2}$. In other words, we choose $\lambda_{2}$ so that the composition of the following chain of morphisms equals $\pi_{2}$ :

$$
\left.\left.\mathbb{F}_{S_{2}} \stackrel{\lambda_{2}}{\rightarrow} \mathbb{F}_{S_{1}} \stackrel{\pi_{1}}{\rightarrow} \mathbb{F}_{S_{1}} /\left\langle E_{1}\right\rangle\right\rangle \stackrel{\theta}{\rightarrow} \mathbb{F}_{S_{2}} /\left\langle E_{2}\right\rangle\right\rangle
$$

Note that such $\lambda_{2}$ exists since $\theta \circ \pi_{1}$ is surjective. Similarly, fix a homomorphism $\lambda_{1}: \mathbb{F}_{S_{1}} \rightarrow \mathbb{F}_{S_{2}}$ satisfying $\pi_{1}=\theta^{-1} \circ \pi_{2} \circ \lambda_{1}$. From now on, in our use of asymptotic $O(\cdot)$-notation, we allow the implied constant to depend on $S_{1}, S_{2}$, $E_{1}$ and $E_{2}$. Since $\lambda_{2}$ and $\lambda_{1}$ have been fixed solely in terms of these four objects, the implied constant is allowed to depend on them as well.

Let $\delta>0$ and $n \in \mathbb{N}$. Let $\Phi_{1}: S_{1} \rightarrow \operatorname{Sym}(n)$ be an $S_{1}$-assignment such that $L_{E_{1}}\left(\Phi_{1}\right) \leq \delta$. Define the $S_{2}$-assignment $\Phi_{2}=\Phi_{1} \circ \lambda_{2}: S_{2} \rightarrow \operatorname{Sym}(n)$. We seek to bound its local defect:

$$
L_{E_{2}}\left(\Phi_{2}\right)=\sum_{w \in E_{2}} d_{n}\left(\Phi_{2}(w), 1\right)=\sum_{w \in E_{2}} d_{n}\left(\Phi_{1}\left(\lambda_{2}(w)\right), 1\right) .
$$

Since $\lambda_{2}(w) \in\left\langle\left\langle E_{1}\right\rangle\right\rangle$ for every $w \in E_{2}$, it follows from Lemma 2.2, applied to $\Phi_{1}$ and the word $\lambda_{2}(w)$, that the $w$-term in the above sum is at most $O\left(L_{E_{1}}\left(\Phi_{1}\right)\right)$. Consequently,

$$
L_{E_{2}}\left(\Phi_{2}\right) \leq C_{1} \cdot L_{E_{1}}\left(\Phi_{1}\right) \leq C_{1} \cdot \delta
$$

for some positive $C_{1}=C_{1}\left(E_{1}, E_{2}\right)$. Hence, there is an $E_{2}$-solution $\Psi_{2}: S_{2} \rightarrow$ $\operatorname{Sym}(n)$ with

$$
d_{n}\left(\Phi_{2}, \Psi_{2}\right) \leq \operatorname{SR}_{E_{2}}\left(L_{E_{2}}\left(\Phi_{2}\right)\right) \leq \operatorname{SR}_{E_{2}}\left(C_{1} \cdot \delta\right)
$$

Let $\Psi_{1}$ be the $S_{1}$-assignment $\Psi_{2} \circ \lambda_{1}: S_{1} \rightarrow \operatorname{Sym}(n)$. Note that $\Psi_{1}$ is an $E_{1}$-solution. Thus, $G_{E_{1}}\left(\Phi_{1}\right) \leq d_{n}\left(\Phi_{1}, \Psi_{1}\right)$. We proceed to bound this distance. By the triangle inequality,

$$
\begin{aligned}
d_{n}\left(\Phi_{1}, \Psi_{1}\right) & \leq d_{n}\left(\Phi_{1}, \Phi_{2} \circ \lambda_{1}\right)+d_{n}\left(\Phi_{2} \circ \lambda_{1}, \Psi_{1}\right) \\
& =d_{n}\left(\Phi_{1}, \Phi_{1} \circ \lambda_{2} \circ \lambda_{1}\right)+d_{n}\left(\Phi_{2} \circ \lambda_{1}, \Psi_{2} \circ \lambda_{1}\right) .
\end{aligned}
$$

We turn to bound both terms of the right hand side. For the first term, note that

$$
\pi_{1}=\theta^{-1} \circ \pi_{2} \circ \lambda_{1}=\theta^{-1} \circ \theta \circ \pi_{1} \circ \lambda_{2} \circ \lambda_{1}=\pi_{1} \circ \lambda_{2} \circ \lambda_{1},
$$

and so $\lambda_{2} \circ \lambda_{1}$ satisfies the requirements of Lemma 2.3. Hence, due to this lemma,

$$
d_{n}\left(\Phi_{1}, \Phi_{1} \circ \lambda_{2} \circ \lambda_{1}\right) \leq O\left(L_{E_{1}}\left(\Phi_{1}\right)\right) \leq O(\delta)
$$


Turning to the second term,

$$
d_{n}\left(\Phi_{2} \circ \lambda_{1}, \Psi_{2} \circ \lambda_{1}\right)=\sum_{s_{2} \in S_{2}} d_{n}\left(\Phi_{2}\left(\lambda_{1}\left(s_{2}\right)\right), \Psi_{2}\left(\lambda_{1}\left(s_{2}\right)\right)\right) .
$$

By Lemma 2.1, applied to $\Phi_{2}, \Psi_{2}$ and the word $\lambda_{1}\left(s_{2}\right)$, the $s_{2}$-term of this sum is upper bounded by $O\left(d_{n}\left(\Phi_{2}, \Psi_{2}\right)\right)$, and so

$$
d_{n}\left(\Phi_{2} \circ \lambda_{1}, \Psi_{2} \circ \lambda_{1}\right) \leq O\left(d_{n}\left(\Phi_{2}, \Psi_{2}\right)\right) \leq O\left(\operatorname{SR}_{E_{2}}\left(C_{1} \cdot \delta\right)\right) .
$$

We conclude that

$$
G_{E}\left(\Phi_{1}\right) \leq d_{n}\left(\Phi_{1}, \Psi_{1}\right) \leq O\left(\mathrm{SR}_{E_{2}}\left(C_{1} \cdot \delta\right)+\delta\right)
$$

Remark 2.4. In Proposition A.5, we show that $\mathrm{SR}_{E}(\delta) \geq \Omega(\delta)$ for every equationset $E$ which is not empty and not $\{1\}$. When $E$ is $\emptyset$ or $\{1\}$, however, it is clear that $\mathrm{SR}_{E} \equiv 0$. This is worth noting, since the free group $\mathbb{F}_{S}$ can be defined by either a trivial equation-set over $S$, or by a certain nontrivial equation-set over some larger finite set of variables. Two nuances in Definitions 1.10 and 1.13 ensure that the stability rate and degree of $\mathbb{F}_{S}$ are well-defined despite this phenomenon. The first is the addition of the term $C \delta$ to the inequalities in Definition 1.10, and the second is the restriction $k \geq 1$ in Equation (1.1) in Definition 1.13.

\section{$3 \quad$ Stability and graphs of actions}

This section reformulates the notions of stability and stability rate in terms of group actions, and provides basic tools arising from this point of view. Before we begin, a small clarification regarding terminology is in order: When a group homomorphism $\theta: \Lambda_{2} \rightarrow \Lambda_{1}$ is fixed and understood from the context, we regard any given $\Lambda_{1}$-set $X$ as a $\Lambda_{2}$-set as well via $\theta$, i.e., for $g_{2} \in \Lambda_{2}$ and $x \in X$, we let $g_{2} \cdot x=\theta\left(g_{2}\right) \cdot x$. In most cases in the sequel, $\Lambda_{1}$ is a group generated by a finite set $S, \Lambda_{2}=\mathbb{F}_{S}$ is a free group on $S$, and $\theta$ is the natural quotient map $\mathbb{F}_{S} \rightarrow \Lambda_{1}$. So, when a $\Lambda_{1}$-set $X$ appears where an $\mathbb{F}_{S}$-set is expected, we treat $X$ as an $\mathbb{F}_{S}$-set in this manner. In some other cases, the role of $\Lambda_{2}$ is taken by a free abelian group, rather than a free group.

\subsection{Stability in terms of group actions}

Throughout Section 3.1, we fix an equation-set $E$ over the finite set of variables $S$, and denote $\Gamma=\mathbb{F}_{S} /\langle\langle E\rangle$. As mentioned in Section 1.1, an $S$-assignment $\Phi: S \rightarrow \operatorname{Sym}(n)$ can also be regarded as a group action of $\mathbb{F}_{S}$ on $[n]$. We write $\mathbb{F}_{S}(\Phi)$ for the $\mathbb{F}_{S}$-set whose set of points is $[n]$, with the group action given by $s \cdot x=\Phi(s)(x)$. We now expand upon this view, rephrasing the definition of local and global defect in terms of group actions, along the same 


\section{ABELIAN GROUPS ARE POLYNOMIALLY STABLE}

lines as in Section 3.2 of [4]. This will enable us to prove our main theorems using a geometric approach, focusing on the geometry of an edge-labeled graph representing $\mathbb{F}_{S}(\Phi)$.

Definition 3.1. Let $X$ be a finite $\mathbb{F}_{S}$-set. The local defect of $X$ with respect to $E$ is

$$
L_{E}(X)=\frac{1}{|X|} \cdot|\{(x, w) \in X \times E \mid w \cdot x \neq x\}| .
$$

It follows directly from the definitions that $L_{E}(\Phi)=L_{E}\left(\mathbb{F}_{S}(\Phi)\right)$ for an assignment $\Phi: S \rightarrow \operatorname{Sym}(n)$.

We turn to the global defect. Let us first characterize $E$-solutions through actions. It is not hard to see that $\Phi: S \rightarrow \operatorname{Sym}(n)$ is an $E$-solution if and only if the action $\mathbb{F}_{S} \curvearrowright \mathbb{F}_{S}(\Phi)$ factors through the group $\Gamma$, that is, if every two elements $u, v \in \mathbb{F}_{S}$, belonging to the same coset in $\mathbb{F}_{S} /\langle\langle E\rangle\rangle$, act on $[n]$ in the same manner. In this case, $\Gamma$ itself acts on $\mathbb{F}_{S}(\Phi)$.

We proceed to define a metric of similarity between $\mathbb{F}_{S}$-sets.

Definition 3.2. Let $X$ and $Y$ be finite $\mathbb{F}_{S}$-sets, where $|X|=|Y|=n$. For a function $f: X \rightarrow Y$, define

$$
\|f\|_{S}=\sum_{s \in S} \frac{1}{n} \cdot|\{(s, x) \in S \times X \mid f(s \cdot x) \neq s \cdot f(x)\}| .
$$

Furthermore, define

$$
d_{S}(X, Y)=\min \left\{\|f\|_{S} \mid f: X \rightarrow Y \text { is a bijection }\right\} .
$$

Note that $d_{S}(X, Y)=0$, if and only if $X$ and $Y$ are isomorphic as $\mathbb{F}_{S}$-sets. Also, for $S$-assignments $\Phi, \Psi: S \rightarrow \operatorname{Sym}(n)$ we have $d_{n}(\Phi, \Psi)=\left\|\operatorname{id}_{\Phi, \Psi}\right\|_{S}$, where $\operatorname{id}_{\Phi, \Psi}: \mathbb{F}_{S}(\Phi) \rightarrow \mathbb{F}_{S}(\Psi)$ is the identity map $[n] \rightarrow[n]$.

We use this metric to express the notion of global defect.

Definition 3.3. Let $X$ be a finite $\mathbb{F}_{S}$-set. The global defect of $X$ with respect to $E$ is

$$
G_{E}(X)=\min \left\{d_{S}(X, Y) \mid Y \text { is a } \Gamma \text {-set and }|Y|=|X|\right\} .
$$

Proposition 3.4. Let $\Phi: S \rightarrow \operatorname{Sym}(n)$ be an $S$-assignment. Then,

$$
G_{E}(\Phi)=G_{E}\left(\mathbb{F}_{S}(\Phi)\right)
$$

Proof. Let $\Psi: S \rightarrow \operatorname{Sym}(n)$ be an $E$-solution which minimizes $d_{n}(\Phi, \Psi)$. Let $Y$ be a $\Gamma$-set, $|Y|=n$, which minimizes $d_{S}\left(\mathbb{F}_{S}(\Phi), Y\right)$. We need to show that $d_{n}(\Phi, \Psi)=d_{S}(\mathbb{F}(\Phi), Y)$. Indeed, on one hand,

$$
d_{S}\left(\mathbb{F}_{S}(\Phi), Y\right) \leq d_{S}\left(\mathbb{F}_{S}(\Phi), \mathbb{F}_{S}(\Psi)\right) \leq\left\|\operatorname{id}_{\Phi, \Psi}\right\|_{S}=d_{n}(\Phi, \Psi)
$$

where the first inequality follows from the defining property of $Y$, and the second from the definition of $d_{S}$. On the other hand, take a bijection $f: \mathbb{F}_{S}(\Phi) \rightarrow Y$ for which $d_{S}\left(\mathbb{F}_{S}(\Phi), Y\right)=\|f\|_{S}$, and define an $S$-assignment $\Theta: S \rightarrow \operatorname{Sym}(n)$ 
by $\Theta(s)(x)=f^{-1}(s \cdot f(x))$. Then, $\Theta$ is an $E$-solution because $Y$ is a $\Gamma$-set, and so

$$
d_{n}(\Phi, \Psi) \leq d_{n}(\Phi, \Theta)=\left\|\operatorname{id}_{\Phi, \Theta}\right\|_{S}=\|f\|_{S}=d_{S}\left(\mathbb{F}_{S}(\Phi), Y\right) .
$$

The above discussion enables us to define the stability rate of $E$ in terms of group actions, as recorded below:

Proposition 3.5. The stability rate of $E$ is given by

$$
\operatorname{SR}_{E}(\delta)=\sup \left\{G_{E}(X) \mid X \text { is a finite } \mathbb{F}_{S} \text { - set and } L_{E}(X) \leq \delta\right\} .
$$

Definition 3.6. For an $\mathbb{F}_{S}$-set $X$, define the set of E-abiding points in $X$ as

$$
X_{E}=\{x \in X \mid \forall w \in E \quad w \cdot x=x\} .
$$

Note that for an $\mathbb{F}_{S}$-set $X$, we have

$$
\frac{\left|X \backslash X_{E}\right|}{|X|} \leq L_{E}(X) \leq|E| \cdot \frac{\left|X \backslash X_{E}\right|}{|X|}
$$

\subsection{Graphs of actions}

As mentioned, it will be useful to represent a group action as a labeled graph. Throughout Section 3.2, we fix a finite set $S$ and a group $\Lambda$ generated by $S$. We have a natural surjection $\mathbb{F}_{S} \rightarrow \Lambda$ which enables us to regard a given action of $\Lambda$ as an action of $\mathbb{F}_{S}$. The action graph of a finite $\Lambda$-set $X$ (with regard to the set of generators $S$ ) is the edge-labeled directed graph over the vertex set $X$, which has a directed edge labeled $s$ from $x$ to $s \cdot x$ for each $x \in X$ and $s \in S$. Note that the action graph of $X$ remains the same if we choose to treat $X$ as an $\mathbb{F}_{S}$-set rather a $\Lambda$-set.

In the context of graphs of actions, it is often useful to consider pointed sets $\left(X, x_{0}\right)$, i.e., a set $X$ together with a distinguished point $x_{0} \in X$. The role of $X$ will always be taken by a $\Lambda$-set or a subset of a $\Lambda$-set. We use the notation $f:\left(X, x_{0}\right) \rightarrow\left(Y, y_{0}\right)$ for a map $f: X \rightarrow Y$ which sends $x_{0} \mapsto y_{0}$.

It is helpful to consider the following definitions with the role of $\Lambda$ taken by the free group $\mathbb{F}_{S}$ itself, or with $\Lambda=\mathbb{Z}^{|S|}$. We proceed to define isomorphisms of subgraphs of action graphs, and several related notions.

Definition 3.7. Let $X$ and $Y$ be $\Lambda$-sets and let $f: X_{0} \rightarrow Y_{0}$ be a map between subsets $X_{0} \subseteq X$ and $Y_{0} \subseteq Y$.

(i) For $s \in S$ and $x \in X_{0}$, we say that $f$ preserves the edge $x \stackrel{s}{\longrightarrow}$ if either $s \cdot x \notin X_{0}$ and $s \cdot f(x) \notin Y_{0}$, or $s \cdot x \in X_{0}, s \cdot f(x) \in Y_{0}$ and $f(s \cdot x)=s \cdot f(x)$.

(ii) If $f$ is bijective, and preserves $x \stackrel{s}{\longrightarrow}$ for every $s \in S$ and $x \in X_{0}$, we say that $f$ is a subgraph isomorphism from $X_{0}$ to $Y_{0}$. 


\section{ABELIAN GROUPS ARE POLYNOMIALLY STABLE}

For a $\Lambda$-set $X$, a point $x \in X$ and a subset $P \subseteq \Lambda$, we write $P \cdot x=$ $\{p \cdot x \mid p \in P\}$. For subsets $P_{1}$ and $P_{2}$ of $\Lambda$, we write $P_{1} \cdot P_{2}=\left\{p_{1} \cdot p_{2} \mid p_{1} \in P_{1}, p_{2} \in P_{2}\right\}$.

Definition 3.8. Let $(X, x)$ and $(Y, y)$ be pointed $\Lambda$-sets and $P \subseteq \Lambda$, a subset. Assume that

$$
\operatorname{Stab}_{\Lambda}(x) \cap\left(P^{-1} P\right) \subseteq \operatorname{Stab}_{\Lambda}(y) \cap\left(P^{-1} P\right)
$$

Define the function $F_{P, x, y}: P \cdot x \rightarrow P \cdot y$ by $F_{P, x, y}(p \cdot x)=p \cdot y$ for each $p \in P$. Note that this function is well-defined since if $p_{1}, p_{2} \in P$ and $p_{1} \cdot x=p_{2} \cdot x$, then $p_{2}^{-1} p_{1} \in \operatorname{Stab}_{\Lambda}(x)$, and so $p_{2}^{-1} p_{1} \in \operatorname{Stab}_{\Lambda}(y)$, hence $p_{1} \cdot y=p_{2} \cdot y$.

In the notation of the above definition, the function $F_{P, x, y}$ is injective if and only if the inclusion in (3.2) is in fact an equality. The special case of Definition 3.8 where $X=\Lambda$ gives rise to the following definition:

Definition 3.9. Let $Y$ be a $\Lambda$-set, $y \in Y$ and $P \subseteq \Lambda$, a subset. We say that $P$ injects into $Y$ at $y$ if the map $F_{P, 1_{\Lambda}, y}: P \rightarrow P \cdot y$ is injective, or, equivalently, if $\operatorname{Stab}_{\Lambda}(y) \cap\left(P^{-1} P\right)=\left\{1_{\Lambda}\right\}$. We say that $P$ bijects onto $Y$ at $y$ if this map is bijective.

Note that Definition 3.9 merely requires the map $F_{P, 1_{\Lambda}, y}$ to be injective, but not necessarily a subgraph isomorphism. We seek sufficient conditions which

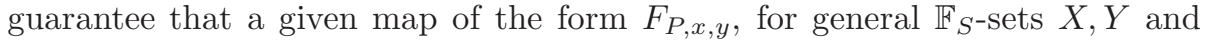
points $x \in X, y \in Y$, preserves a given edge, or even that it is a subgraph isomorphism. The following two lemmas provide such conditions by considering short elements of $\Lambda$ and whether or not they belong to $\operatorname{Stab}_{\Lambda}(x)$ and $\operatorname{Stab}_{\Lambda}(y)$.

Lemma 3.10. Let $(X, x)$ and $(Y, y)$ be pointed $\Lambda$-sets and $P \subseteq \Lambda$, a subset, such that Condition (3.2) of Definition 3.8 is satisfied. Let $s \in S, p \in P$ and assume that

$$
\operatorname{Stab}_{\Lambda}(x) \cap\left(P^{-1} \cdot s p\right)=\operatorname{Stab}_{\Lambda}(y) \cap\left(P^{-1} \cdot s p\right) .
$$

Then, the map $F=F_{P, x, y}: P \cdot x \rightarrow P \cdot y$ preserves $p \cdot x \stackrel{s}{\longrightarrow}$.

Proof. We first note that for $p_{1} \in P$,

$$
s p \cdot x=p_{1} \cdot x \quad \text { if and only if } \quad s p \cdot y=p_{1} \cdot y \text {. }
$$

Indeed, $s p \cdot x=p_{1} \cdot x$ if and only if $p_{1}^{-1} s p \in \operatorname{Stab}_{\Lambda}(x)$ if and only if $p_{1}^{-1} s p \in$ $\operatorname{Stab}_{\Lambda}(y)$ if and only if $s p \cdot y=p_{1} \cdot y$.

It follows from $(3.3)$ that $s \cdot(p \cdot x)$ belongs to $P \cdot x$ if and only if $s \cdot F(p \cdot x)=$ $s \cdot(p \cdot y)$ belongs to $P \cdot y$. Assume that these equivalent conditions hold. It remains to show that in this case, $F(s \cdot(p \cdot x))=s \cdot F(p \cdot x)$. Write $s p \cdot x=p_{1} \cdot x$ for $p_{1} \in P$. By $(3.3), s p \cdot y=p_{1} \cdot y$. So, $F(s \cdot(p \cdot x))=F\left(p_{1} \cdot x\right)=p_{1} \cdot y=$ $s p \cdot y=s \cdot F(p \cdot x)$, as required. 
Lemma 3.11. Let $(X, x)$ and $(Y, y)$ be pointed $\Lambda$-sets and $P \subseteq \Lambda$, a subset. Write $S_{1}=S \cup\left\{1_{\Lambda}\right\}$ and assume that

$$
\operatorname{Stab}_{\Lambda}(x) \cap\left(P^{-1} \cdot S_{1} \cdot P\right)=\operatorname{Stab}_{\Lambda}(y) \cap\left(P^{-1} \cdot S_{1} \cdot P\right) .
$$

Then, $F_{P, x, y}: P \cdot x \rightarrow P \cdot y$ is well-defined and is a subgraph isomorphism.

Proof. Since $\operatorname{Stab}_{\Lambda}(x) \cap\left(P^{-1} \cdot P\right)=\operatorname{Stab}_{\Lambda}(y) \cap\left(P^{-1} \cdot P\right)$, the map $F_{P, x, y}$ is well-defined and injective. Furthermore, for each $s \in S$ and $x \in P \cdot x$, since $\operatorname{Stab}_{\Lambda}(x) \cap\left(P^{-1} \cdot s p\right)=\operatorname{Stab}_{\Lambda}(y) \cap\left(P^{-1} \cdot s p\right)$, Lemma 3.10 implies that $F_{P, x, y}$ preserves $x \stackrel{s}{\longrightarrow}$.

We proceed to define balls in $\mathbb{F}_{S}$ and $\mathbb{F}_{S}$-sets, and give a useful corollary of Lemma 3.11. The word norm $|\cdot|$ on $\mathbb{F}_{S}$ in defined for a word $w \in \mathbb{F}_{S}$ as the length of $w$ when written as a reduced word over $S^{ \pm}$. Let $X$ be an $\mathbb{F}_{S^{-}}$set. The word-norm induces a metric $d_{X}$ on $X$ :

$$
\forall x_{1}, x_{2} \in X \quad d_{X}\left(x_{1}, x_{2}\right)=\min \left\{|w| \mid w \in \mathbb{F}_{S}, w \cdot x_{1}=x_{2}\right\} .
$$

Write $B_{X}(x, r)$ for the ball of radius $r \geq 0$ centered at the point $x \in X$ with respect to $d_{X}$. For $A \subseteq X$, let $B(A, r)=\bigcup_{x \in A} B(x, r)$. In the special case $X=\Lambda$, we also write $B_{\Lambda}(r)$ for $B_{\Lambda}\left(1_{\Lambda}, r\right)$. This notation will be used often with either $\Lambda=\mathbb{F}_{S}$ or $\Lambda=\mathbb{Z}^{|S|}$. For $r \geq 0$, plugging in $P=B_{\Lambda}(r)$ in Lemma 3.11 , we deduce the following corollary:

Lemma 3.12. Let $(X, x)$ and $(Y, y)$ be $\Lambda$-sets and $r \geq 0$, an integer. Assume that

$$
\operatorname{Stab}_{\Lambda}(x) \cap B_{\Lambda}(2 r+1)=\operatorname{Stab}_{\Lambda}(y) \cap B_{\Lambda}(2 r+1) .
$$

Then, the map $F_{B_{\Lambda}(r), x, y}: B_{X}(x, r) \rightarrow B_{Y}(y, r)$ is well-defined and is a subgraph isomorphism.

Now, assume that $E \subseteq \mathbb{F}_{S}$ is a finite set generating the kernel of the surjection $\mathbb{F}_{S} \rightarrow \Lambda$ as a normal subgroup (hence $\mathbb{F}_{S} /\langle\langle E\rangle \cong \Lambda$ ). We end this section with two definitions and a basic lemma that allows us to bound the global defect $G_{E}(X)$ of an $\mathbb{F}_{S}$-set $X$ with respect to $E$ (see Definition 3.3) in terms of properties of a map between graphs of actions.

Definition 3.13. Let $X$ be a $\Lambda$-set and $X_{0} \subseteq X$ a subset. A point $x \in X_{0}$ is internal in $X_{0}$ if $S \cdot x \subseteq X_{0}$.

Definition 3.14. Let $X$ and $Y$ be $\Lambda$-sets and $X_{0} \subseteq X$, a subset. Take a function $f: X_{0} \rightarrow Y$. Define the set $\mathrm{Eq}(f) \subseteq X$ of equivariance points of $f$ as

$$
\mathrm{Eq}(f)=\left\{x \in X_{0} \mid \forall s \in S \quad s \cdot x \in X_{0} \text { and } f(s \cdot x)=s \cdot f(x)\right\} .
$$

That is, $\mathrm{Eq}(f)$ is the set of internal points $x \in X_{0}$, for which $f$ preserves $x \stackrel{s}{\longrightarrow}$ for all $s \in S$.

Lemma 3.15. Let $Y$ be a $\Lambda$-set, $X$ an $\mathbb{F}_{S}$-set and $f: Y \rightarrow X$ an injective map. Then, $G_{E}(X) \leq|S| \cdot\left(1-\frac{1}{|X|} \cdot|\operatorname{Eq}(f)|\right)$. 
Proof. Let $Z$ be a trivial $\Lambda$-set of cardinality $|Y|-|X|$, i.e., each $g \in \Lambda$ fixes each $z \in Z$. Fix a bijection $f_{Z}: Z \rightarrow X \backslash \operatorname{Im}(f)$. We consider the disjoint union $Y \amalg Z$ and the bijection $f \amalg f_{Z}: Y \amalg Z \rightarrow X$. Then,

$$
\begin{aligned}
G_{E}(X) & \leq d_{S}(Y \coprod Z, X) \\
& \leq\left\|f \coprod f_{Z}\right\|_{S} \\
& \leq \frac{1}{|X|} \cdot\left(|X| \cdot|S|-|S| \cdot\left|\operatorname{Eq}\left(f \coprod f_{Z}\right)\right|\right) \\
& \leq \frac{1}{|X|} \cdot\left(|X| \cdot|S|-|S| \cdot|\operatorname{Eq}(f)|-|S| \cdot\left|\operatorname{Eq}\left(f_{Z}\right)\right|\right) \\
& \leq|S| \cdot\left(1-\frac{1}{|X|} \cdot|\operatorname{Eq}(f)|\right) .
\end{aligned}
$$

\section{Abelian groups are polynomially stable}

The aim of this section is to prove Theorem 1.16, our main theorem. We begin by defining several objects that shall remain fixed throughout Section 4 .

Let $\Gamma$ be a finitely-generated abelian group. Without loss of generality we can realize $\Gamma$ as follows: Let $m \geq d \geq 0$, take a basis $\left\{e_{1}, \ldots, e_{m}\right\}$ for $\mathbb{Z}^{m}$ and let $2 \leq \beta_{m-d+1} \leq \ldots \leq \beta_{m}$ be integers. Define

$$
K=\left\langle\left\{\beta_{i} \cdot e_{i}\right\}_{i=m-d+1}^{m}\right\rangle \leq \mathbb{Z}^{m}
$$

and write $\Gamma=\mathbb{Z}^{m} / K$. Let $\operatorname{Tor}(\Gamma)$ denote the torsion subgroup of $\Gamma$. Let

$$
\beta_{E}= \begin{cases}\beta_{m} & \text { if } m>d \\ 1 & \text { if } m=d .\end{cases}
$$

Theorem 1.16 asserts that $\operatorname{deg}\left(\mathrm{SR}_{\Gamma}\right)$ is finite. We will, in fact, provide an explicit upper bound on $\operatorname{deg}\left(\mathrm{SR}_{\Gamma}\right)$. In the case $d=0$, i.e., if $\Gamma$ is finite, Proposition A.4 says that $\operatorname{deg}\left(\mathrm{SR}_{\Gamma}\right)=1$. We proceed assuming that $d \geq 1$. We shall show that

$$
\operatorname{deg}\left(\mathrm{SR}_{\Gamma}\right) \leq C_{\text {bound }}(\Gamma)
$$

for

$$
C_{\text {bound }}(\Gamma)=O\left(2^{d} \cdot d \cdot \max \left\{d \log d, \log \beta_{E}, 1\right\}\right),
$$

where the implied constant of the $O(\cdot)$ notation is an absolute constant. We note that, in order to minimize $C_{\text {bound }}(\Gamma)$, one may let $\beta_{m-d+1}, \ldots, \beta_{m}$ correspond to the primary decomposition of $\Gamma$, thus making the constant $\beta_{E}$ the largest prime power in that decomposition.

Let $\mathbb{F}_{m}$ be the free group on $S=\left\{\hat{e}_{1}, \ldots, \hat{e}_{m}\right\}$, and consider the surjection $\pi: \mathbb{F}_{m} \rightarrow \mathbb{Z}^{m}$, sending $\hat{e}_{i} \mapsto e_{i}$. We get a sequence of surjections

$$
\mathbb{F}_{m} \stackrel{\pi}{\longrightarrow} \mathbb{Z}^{m} \longrightarrow \Gamma \text {. }
$$


We also fix a free group $\mathbb{F}_{d}$, generated by $\left\{\hat{e}_{1}, \ldots, \hat{e}_{d}\right\}$. That is, we write $\mathbb{F}_{d}$ for this fixed copy of a free group of rank $d$ inside our fixed free group $\mathbb{F}_{m}$.

By the definition, the stability rate $\mathrm{SR}_{\Gamma}$ of $\Gamma$ can be computed through any presentation of $\Gamma$ (see Proposition 1.11). We proceed to choose the equation-set $E$, defining $\Gamma$, with which we will work. Let

$$
E_{0}=\left\{\left[\hat{e}_{i}^{\epsilon_{1}}, \hat{e}_{j}^{\epsilon_{2}}\right] \in \mathbb{F}_{m} \mid i, j \in[m], \quad \epsilon_{1}, \epsilon_{2} \in\{+1,-1\}\right\} \subseteq \mathbb{F}_{m},
$$

where $[x, y]$ denotes the commutator $x y x^{-1} y^{-1}$, and define

$$
E=E_{0} \cup\left\{\hat{e}_{i}^{\beta_{i}} \mid m-d+1 \leq i \leq m\right\} \subseteq \mathbb{F}_{m} .
$$

Note that if $d=m$, then $E$ is equivalent to the equation-set $E_{\text {comm }}^{d}$ from the introduction. Since $\Gamma \cong \mathbb{F}_{m} /\langle\langle E\rangle\rangle$, we have $\mathrm{SR}_{\Gamma}=\left[\mathrm{SR}_{E}\right]$, and so our goal is to prove that

$$
\operatorname{deg}\left(\mathrm{SR}_{E}\right) \leq C_{\text {bound }}(\Gamma) .
$$

For future reference, we fix the following constants:

$$
\begin{aligned}
C_{d} & =\max \left\{3 \cdot 7^{d} \cdot d^{2 d+2}, \beta_{E}\right\} \\
t_{E} & =\max \left\{d^{-1} \cdot(m-d) \cdot m \cdot \beta_{E}, 2\right\} .
\end{aligned}
$$

Additionally, we let

$$
\hat{T}=\left\{\prod_{i=m-d+1}^{m} \hat{e}_{i}^{\alpha_{i}} \in \mathbb{F}_{m} \mid \forall i 0 \leq \alpha_{i}<\beta_{i}\right\},
$$

and

$$
T=\left\{\sum_{i=m-d+1}^{m} \alpha_{i} \cdot e_{i} \in \mathbb{Z}^{m} \mid \forall i 0 \leq \alpha_{i}<\beta_{i}\right\} .
$$

In the rest of this section, the implied constants in the $O(\cdot)$ notation are allowed to depend on $m, d$ and $E$.

\subsection{Proof plan}

In this section we outline our proof of Theorem 1.16, as implemented in Sections 4.2-4.7.

Let $X$ be an $\mathbb{F}_{m}$-set, and write $n=|X|$. By Proposition 3.5, in order to bound $\mathrm{SR}_{E}$, it suffices to bound $G_{E}(X)$ in terms of $L_{E}(X)$. To this end, we algorithmically construct a $\Gamma$-set $Y$ (Proposition 4.30), together with a certain injection $f: Y \rightarrow X$ with many equivariance points, namely, $|\mathrm{Eq}(f)| \geq n$. $\left(1-O\left(L_{E}(X)^{\frac{1}{C_{\text {bound }}(\Gamma)}}\right)\right)$. Lemma 3.15 then gives the bound $G_{E}(X) \leq|S|$. $\left(1-\frac{|\mathrm{Eq}(f)|}{n}\right) \leq O\left(L_{E}(X)^{\frac{1}{C_{\mathrm{bound}}(\Gamma)}}\right)$, which yields the claim of Theorem 1.16.

We build $Y$ as the disjoint union of a collection of small $\Gamma$-sets $\left\{Y_{x}\right\}_{x \in J}$, each equipped with an injection $f_{x}: Y_{x} \rightarrow X$. The images of these injections are 


\section{ABELIAN GROUPS ARE POLYNOMIALLY STABLE}

pairwise disjoint and $f: Y \rightarrow X$ is taken to be the disjoint union of the maps $f_{x}$. Clearly, due to this construction, $|\mathrm{Eq}(f)|$ is at least $\sum_{x \in J}\left|\mathrm{Eq}\left(f_{x}\right)\right|$, so we wish to maximize the latter sum. Towards this end, it is desirable that the images of the injections $f_{x}$ cover almost all of $X$, and that each of the injections has a large fraction of equivariance points. We manage to construct the injections $f_{x}$ so that the equivariance points of $f_{x}$ are approximately those points in $Y_{x}$ that are mapped to internal points of $\operatorname{Im}\left(f_{x}\right) \subseteq X$. Hence, we think of the ratio $\frac{\left|Y_{x} \backslash \mathrm{Eq}\left(f_{x}\right)\right|}{\left|Y_{x}\right|}$ as an isoperimetric ratio, which we wish to minimize.

The reader may prefer to read both the proof and its outline under the simplifying assumption that $\Gamma$ is torsion-free, i.e., $m=d$ and, accordingly, $\Gamma=\mathbb{Z}^{m}$. In fact, the torsion-free case of Theorem 1.16 implies the general case. This follows from Proposition A.3, since every finitely-generated abelian group is a quotient of a torsion-free finitely-generated abelian group by a finitelygenerated subgroup. The simplified strategy, of starting with the torsion-free case and then using Proposition A.3, comes at the price of a somewhat worse bound on the degree, compared to (4.2).

We turn to give an outline of our algorithm.

\subsubsection{The algorithm constructing $Y$ and $f$}

Our algorithm works iteratively as follows. We first initialize a rather large number $t_{1}$ which depends on the ratio $\frac{\left|X_{E}\right|}{|X|}$, which in turn is related to $L_{E}(X)$ (see Equation (3.1)). In the first iteration, we find, in a greedy manner, a collection of $\Gamma$-sets $\left\{Y_{x}\right\}_{x \in J_{1}}$ and respective injections $\left\{f_{x}: Y_{x} \rightarrow X\right\}_{x \in J_{1}}$ with pairwise disjoint images, such that $\frac{\left|Y_{x} \backslash \mathrm{Eq}\left(f_{x}\right)\right|}{\left|Y_{x}\right|} \leq O\left(\frac{1}{t_{1}}\right)$ for each $x \in J_{1}$. We think of the images of the injections $\left\{f_{x}\right\}_{x \in J_{1}}$ as "tiles" embedded in $X$, and of $t_{1}$ as a parameter used in the construction of these tiles. The set $J_{1}$ is maximal in the sense that we cannot add more tiles with parameter $t_{1}$ without violating the constraint that they be disjoint. We proceed to tile the remainder of $X$. We define a new parameter $t_{2}<t_{1}$ which is equal to $t_{1}$ divided by some constant, and repeat this process for another iteration, which yields additional $\Gamma$ sets $\left\{Y_{x}\right\}_{x \in J_{2}}$ and corresponding injections, perhaps with a worse isoperimetric ratio. We require that the images of these injections be disjoint from each other, as well as from the images obtained in the previous iteration. We proceed in this manner, tiling a constant fraction of the remainder of $X$ in each iteration, until $t_{i}$ is below a certain threshold, at which point the iterative algorithm halts. Finally, we set $Y$ to be the disjoint union of the $\Gamma$-sets $\left\{Y_{x}\right\}_{x \in J_{1} \cup \cdots \cup J_{s}}$ constructed throughout the $s$ iterations, and define $f: Y \rightarrow X$ as the disjoint union of the maps $\left\{f_{x}\right\}_{x \in J_{1} \cup \ldots \cup J_{s}}$.

Note that, as the algorithm progresses, our injections become less and less efficient, that is, their images have a larger isoperimetric ratio. After developing the necessary machinery in Sections 4.4-4.6, we conclude the proof of Theorem 1.16 in Section 4.7 by defining the above algorithm, and showing that it produces an injection $f$ with many equivariance points. We turn to discuss the technique by which we build the sets $Y_{x}$ and the corresponding injections $f_{x}$. 


\subsubsection{Mapping a single $\Gamma$-set $Y_{x}$ into $X$}

As mentioned, the $i$-th iteration of our algorithm injects a collection of $\Gamma$-sets $\left\{Y_{x}\right\}_{x \in J_{i}}$ into $X$. The isoperimetric ratio of each of these injections must be bounded by $O\left(\frac{1}{t_{i}}\right)$, where $t_{i}$ is the parameter introduced in Section 4.1.1. We now focus on the main technical challenge, namely, building a single finite $\Gamma$-set $Y_{x}$ and a corresponding injection $f_{x}: Y_{x} \rightarrow X$. The efficiency of our construction is reflected in the fact that an "accumulation" of no more than $O\left(t_{i}^{d}\right)$ nearby points of $X$ which belong to $X_{E}$ suffices to construct an injection with isoperimetric constant bounded by $O\left(\frac{1}{t_{i}}\right)$. We begin by describing two essential tools for the construction of $Y_{x}$ and $f_{x}$ :

Tool A (Proposition 4.21): This tool requires a point $x \in X_{E}$ with a large enough neighborhood (called a "box-neighborhood of side-length $t_{i}$ ") which is entirely contained in $X_{E}$. It provides a radius $r_{A} \geq \Omega\left(t_{i}\right)$ such that the ball $B_{X}\left(x, r_{A}\right)$ is contained in the aforementioned neighborhood. It also provides a new (usually infinite) pointed $\Gamma$-set $\left(U_{A}, u_{A}\right)$ and a subgraph isomorphism $f_{A}$ :

$$
\begin{array}{cc}
B_{U_{A}}\left(u_{A}, r_{A}\right) \stackrel{f_{A}}{\longrightarrow} B_{X}\left(x, r_{A}\right) \\
\subseteq & \subseteq \\
\left(U_{A}, u_{A}\right) & X
\end{array}
$$

Tool C (Proposition 4.11): Given a pointed $\Gamma$-set $(V, v)$ and $t_{C} \in \mathbb{N}$, this tool creates an injective map:

$$
(Y, y) \stackrel{f_{C}}{\longrightarrow}(V, v)
$$

where $(Y, y)$ is a new small finite pointed $\Gamma$-set and the isoperimetric ratio of $\operatorname{Im}\left(f_{C}\right)$ is bounded by $O\left(\frac{1}{t_{C}}\right)$.

One may be tempted to try to construct $Y_{x}$ and the injective map $f_{x}: Y_{x} \rightarrow$ $X$ as follows: Locate a point $x \in X_{E}$ with the property required by Tool $\mathrm{A}$, and use this tool to create:

$$
B_{U_{A}}\left(u_{A}, r_{A}\right) \stackrel{f_{A}}{\longrightarrow} X
$$

as in the description of Tool A. Then, apply Tool $\mathrm{C}$ to $(V, v)=\left(U_{A}, u_{A}\right)$ with some $t_{C} \geq \Omega\left(t_{i}\right)$, and create:

$$
\left(Y_{x}, y_{x}\right) \stackrel{f_{C}}{\longrightarrow}\left(U_{A}, u_{A}\right)
$$

as in the description of Tool C. Now, in the very fortunate case where the image of $f_{C}$ is contained in the domain $B_{U_{A}}\left(u_{A}, r_{A}\right)$ of $f_{A}$, we can define the map $f_{x}: Y_{x} \rightarrow X$ as the following composition:

$$
Y_{x} \stackrel{f_{C}}{\longrightarrow} B_{U_{A}}\left(u_{A}, r_{A}\right) \stackrel{f_{A}}{\longrightarrow} X .
$$




\section{ABELIAN GROUPS ARE POLYNOMIALLY STABLE}

When this works, the map $f_{x}$ is injective and its image has a small isoperimetric ratio, as required, because these properties hold for $f_{C}$ and since $f_{A}$ : $B_{U_{A}}\left(u_{A}, r_{A}\right) \rightarrow B_{X}\left(x, r_{A}\right)$ is a subgraph isomorphism. However, the image of the map $f_{C}$, produced by Tool $\mathrm{C}$, is usually too large to be contained in the domain of $f_{A}$. We solve this issue by introducing yet another $\Gamma$-set $U_{B}$ which sits between $Y_{x}$ and $B_{U_{A}}\left(u_{A}, r_{A}\right)$ in the above diagram. As is the case for $U_{A}$, the set $U_{B}$ is usually infinite. It is generated by Tool $\mathrm{B}$ (see below), and has a useful combination of properties: (I) locally, $U_{B}$ looks like $U_{A}$ in a rather large radius, and (II) when Tool $\mathrm{C}$ is applied to $U_{B}$, the image in $U_{B}$ of the resulting injection $f_{C}$ is relatively small.

Tool B (Proposition 4.13): Given a pointed $\Gamma$-set $\left(U_{A}, u_{A}\right)$ and $t_{B} \in \mathbb{N}$, this tool creates a subgraph isomorphism:

$$
\begin{aligned}
& \left(U_{B}^{0}, u_{B}\right) \stackrel{f_{B}}{\longrightarrow}\left(U_{A}^{0}, u_{A}\right) \\
& \subseteq \quad \subseteq \\
& \left(U_{B}, u_{B}\right) \quad\left(U_{A}, u_{A}\right)
\end{aligned}
$$

where $\left(U_{B}, u_{B}\right)$ is a new pointed $\Gamma$-set and $U_{A}^{0}$ and $U_{B}^{0}$ are finite sets. The tool guarantees the following properties:

(I) $U_{A}^{0} \subseteq B_{U_{A}}\left(u_{A}, O\left(t_{B}\right)\right)$, and

(II) The set $U_{B}^{0}$ is exactly the image of the map $f_{C}$ that our implementation of Tool $\mathrm{C}$ provides when it is applied to $(V, v)=\left(U_{B}, u_{B}\right)$ with $t_{C}=t_{B}$.

Using all three tools, we define $f_{x}$ as the composition of the following chain of maps:

$$
\begin{array}{cc}
Y_{x} \stackrel{f_{C}}{\longrightarrow} \operatorname{Im}\left(f_{C}\right) \stackrel{f_{B}}{\longrightarrow} B_{U_{A}}\left(u_{A}, r_{A}\right) \stackrel{f_{A}}{\longrightarrow} X \\
\subseteq & \subseteq \\
U_{B} & U_{A}
\end{array}
$$

The objects and maps in the diagram above are created by first using Tool A to create $U_{A}$ and $f_{A}$, then applying Tool $\mathrm{B}$ to $U_{A}$ to create $U_{B}$ and $f_{B}$, and finally applying Tool $\mathrm{C}$ to $U_{B}$ to create $Y_{x}$ and $f_{C}$. All three maps $f_{A}$, $f_{B}$ and $f_{C}$ are injective, and so the same is true for $f_{x}$. Both $f_{A}$ and $f_{B}$ are subgraph isomorphisms onto their respective images, and the image of $f_{C}$ has isoperimetric ratio at most $O\left(\frac{1}{t_{i}}\right)$. Hence, the same isoperimetric property is true for $f_{x}$, as required.

The proofs for Tools A and B use basis reduction theory of sublattices of $\mathbb{Z}^{m}$, and the proof for Tool $\mathrm{C}$ is also in a related spirit. More specifically, a transitive 
$\Gamma$-set $V$ is isomorphic to $\mathbb{Z}^{m} / H$ for some subgroup $H \leq \mathbb{Z}^{m}$, and we are able to study $V$ by applying reduction theory to $H$, thought of as a sublattice of $\mathbb{Z}^{m}$.

We note that the isoperimetric property possessed by each tile serves two purposes in our proof. The "local purpose" is to ensure that each injection $f_{x}$ has a large fraction of equivariance points as described above. The "global purpose" is to ensure that we can pack many tiles into $X$.

\subsection{Geometric definitions}

Section 3.2 introduced the word-norm on a free group, the word-metric on sets which are acted on by a free group, and defined balls with respect to the wordmetric. In addition to these, our proof will make use of various norms on $\mathbb{Z}^{m}$ (specifically, $L^{1}, L^{2}$ and $L^{\infty}$ ), and of "boxes" in free groups. These are described below:

Geometry of $\mathbb{Z}^{m}$ For $1 \leq p \leq \infty$, let $\|\cdot\|_{p}$ denote the $L^{p}$ norm, restricted to $\mathbb{Z}^{m}$. Let $B_{\mathbb{Z}^{m}}^{L^{p}}(x, r) \subseteq \mathbb{Z}^{m}$ be the closed ball, with respect to $\|\cdot\|_{p}$, of radius $r$, centered at $x$. Again, we omit $x$ for a ball centered at $0_{\mathbb{Z}^{m}}$. We note that in the case $p=1,\|\cdot\|_{1}$ coincides with the word-metric on $\mathbb{Z}^{m}$ as an $\mathbb{F}_{m}$-set, and so $B_{\mathbb{Z}^{m}}(x, r)=B_{\mathbb{Z}^{m}}^{L^{1}}(x, r)$.

Boxes in $\mathbb{F}_{m}$ and $\mathbb{F}_{d}$ Let $\mathbb{F}_{k}$ be the free group on $\left\{\hat{e}_{1}, \ldots, \hat{e}_{k}\right\}$ (we are interested in $k \in\{m, d\})$. We say that a word in $\mathbb{F}_{k}$ is sorted if it is of the form $\prod_{i=1}^{k} \hat{e}_{i}^{a_{i}}, a_{i} \in \mathbb{Z}$. Write $\pi_{k}: \mathbb{F}_{k} \rightarrow \mathbb{Z}^{k}$ for the surjection sending $\hat{e}_{i} \mapsto e_{i}$. For each $v \in \mathbb{Z}^{k}$ we define a canonical representative $\hat{v} \in \mathbb{F}_{k}$ of the set $\pi_{k}^{-1}(v)$, namely, $\hat{v}$ is the unique sorted word such that $\pi_{k}(\hat{v})=v$. Reusing this notation, for $w \in \mathbb{F}_{k}$ let $\hat{w}$ denote $\widehat{\pi(w)}$, i.e, the sorted form of $w$. The following definition introduces a key player in our proof:

Definition 4.1. For $t \in \mathbb{N}$, let

$$
\operatorname{Box}_{\mathbb{F}_{k}}(t)=\left\{\hat{v} \mid v \in B_{\mathbb{Z}^{k}}^{L^{\infty}}(t)\right\}=\left\{\hat{v} \mid v \in \mathbb{Z}^{k},\|v\|_{\infty} \leq t\right\} \subseteq \mathbb{F}_{k} .
$$

Note that $w \in \operatorname{Box}_{\mathbb{F}_{k}}(|w|)$ whenever $w$ is sorted. Finally, for a subset $A$ of $\mathbb{Z}^{k}$ or $\mathbb{F}_{k}$, define $\hat{A}=\{\hat{a} \mid a \in A\}$.

\subsection{A review of reduction theory (of sublattices of $\mathbb{Z}^{l}$ )}

Let $l \geq 1$. We use the term lattice interchangeably with a subgroup $H \leq \mathbb{Z}^{l}$. A basis for $H$ is a set of linearly independent vectors $\mathcal{D} \subseteq \mathbb{Z}^{l}$ such that $H$ is the set of all integer combinations of elements in $\mathcal{D}$. It is well known that every lattice affords a basis, and that different bases representing the same lattice all have the same cardinality. Hence, we may define the rank of $H$ by $\operatorname{rank} H=|\mathcal{D}|$. The goal of reduction theory (See [15] for a survey) is to represent $H$ via a reduced basis, namely, a basis consisting of relatively short vectors. In this section, we adapt a certain result from reduction theory to our purposes. 
Definition 4.2. Let $H \leq \mathbb{Z}^{l}$ be a lattice and $k=\operatorname{rank} H$. The successive minima sequence $\lambda_{1}(H), \ldots, \lambda_{k}(H)$ is defined as follows: $\lambda_{i}(H)$ is the minimum radius $r$ for which $\operatorname{rank}\left\langle\left\{v \in H \mid\|v\|_{2} \leq r\right\}\right\rangle \geq i$.

We note that for $l>4$, the vectors that yield the successive minima are not necessarily a basis for $H$ ([16] p. 51). However, as we now elaborate, $H$ does accommodate a basis consisting of vectors within the same order of magnitude as the successive minima. It is known that every lattice has a basis of a certain type called Korkin-Zolotarev reduced [15]. The following proposition about such bases is a part of Theorem 2.1 in [14].

Proposition 4.3. If $H \leq \mathbb{Z}^{l}$ is a lattice and $B=\left\{b_{1}, \ldots, b_{k}\right\}$ is a KorkinZolotarev reduced basis for $H$, then $\left\|b_{i}\right\|_{2} \leq \frac{1}{2} \cdot \sqrt{i+3} \cdot \lambda_{i}(H)$ for each $1 \leq i \leq k$.

Our use of Proposition 4.3 will always be mediated through Proposition 4.5, below.

Definition 4.4. For a finite subset $A \subseteq \mathbb{Z}^{l}$, write $\|A\|_{1}=\sum_{v \in A}\|v\|_{1}$.

Proposition 4.5. Let $H \leq \mathbb{Z}^{l}$ be a lattice of rank $k$ and $t \in \mathbb{N}$, such that $\operatorname{rank}\left\langle H \cap B_{\mathbb{Z}^{l}}^{L^{2}}(t)\right\rangle=k$. Then, $H$ has a basis $\mathcal{D}$ such that:

(i) $\mathcal{D} \subseteq B_{\mathbb{Z}^{l}}(l \cdot t)$.

(ii) $\|\mathcal{D}\|_{1} \leq l^{2} \cdot t$.

Proof. Let $\mathcal{D}=\left\{b_{1}, \ldots, b_{k}\right\}$ be a Korkin-Zolotarev reduced basis for $H$. By Proposition 4.3,

$$
\left\|b_{i}\right\|_{2} \leq \frac{1}{2} \sqrt{i+3} \cdot \lambda_{i}(H) \leq \frac{1}{2} \sqrt{l+3} \cdot t \leq \frac{1}{2} \sqrt{4 l} \cdot t=\sqrt{l} \cdot t
$$

and so

$$
\left\|b_{i}\right\|_{1} \leq l \cdot t
$$

which yields the first claim. The second claim follows since

$$
\|\mathcal{D}\|_{1} \leq|\mathcal{D}| \cdot \max _{1 \leq i \leq k}\left\|b_{i}\right\|_{1} \leq k \cdot l \cdot t \leq l^{2} \cdot r
$$

\subsection{Tool C: The standard-completion isoperimetric method}

In this section we develop Tool $C$. Let $\left(V, v_{0}\right)$ be a pointed $\Gamma$-set and $t \in \mathbb{N}$. Our goal is to build a small finite pointed $\Gamma$-set $\left(Y, y_{0}\right)$, and an injective map $f_{C}:\left(Y, y_{0}\right) \rightarrow\left(V, v_{0}\right)$ such that

$$
\left|\mathrm{Eq}\left(f_{C}\right)\right| \geq|Y| \cdot\left(1-O\left(\frac{1}{t}\right)\right) .
$$




\section{OREN BECKER AND JONATHAN MOSHEIFF}

We may assume that $V$ is transitive, since otherwise we may consider only the component containing $v_{0}$. Hence, $V$ is realizable as $\mathbb{Z}^{m} / H$ for some $K \leq$ $H \leq \mathbb{Z}^{m}$ (see Equation (4.1)), with $v_{0}$ corresponding to the coset $0+H$. Let $\mathcal{D}_{0}$ be a lattice basis for $H$. We first consider the simple case where $\mathcal{D}_{0}$ consists of axis-parallel vectors, namely, $\mathcal{D}_{0}=\left\{\alpha_{i} \cdot e_{i} \mid i \in[m] \backslash I\right\}$ for some $I \subseteq[m]$, where $\left\{\alpha_{i}\right\}_{i \in[m] \backslash I}$ are positive integers. We complete $\mathcal{D}_{0}$ to a full-rank basis $\mathcal{T}=\mathcal{D}_{0} \cup\left\{2 t \cdot e_{i}\right\}_{i \in I}$. Define $\left(Y, y_{0}\right)=\left(\mathbb{Z}^{m} /\langle\mathcal{T}\rangle, 0+\langle\mathcal{T}\rangle\right)$, and note that $Y$ is a $\Gamma$-set since $K \subseteq H \subseteq\langle\mathcal{T}\rangle$.

Each point $y \in Y$ has a unique representation as

$$
y=\sum_{f \in \mathcal{D}_{0}} a_{f} \cdot f+\sum_{i \in I} b_{i} \cdot e_{i}+\langle\mathcal{T}\rangle \quad 0 \leq a_{f}<1,-t \leq b_{i}<t .
$$

Let $f_{C}:\left(Y, y_{0}\right) \rightarrow\left(V, v_{0}\right)$ map such a point $y$ to the point

$$
\sum_{f \in \mathcal{D}_{0}} a_{f} \cdot f+\sum_{i \in I} b_{i} \cdot e_{i}+H
$$

of $V$. This map $f_{C}$ is clearly injective. Now, consider a point $y \in Y$ as above. The map $f_{C}$ necessarily preserves the edge $y \stackrel{e_{i}}{\longrightarrow}$ for each $i \in[m] \backslash I$. If $i \in I$, then $f_{C}$ preserves this edge unless $b_{i}=t-1$. It follows that

$\operatorname{Eq}\left(f_{C}\right)=\left\{\sum_{f \in \mathcal{D}_{0}} a_{f} \cdot f+\sum_{i \in I} b_{i} \cdot e_{i}+\langle\mathcal{T}\rangle \mid 0 \leq a_{f}<1\right.$ and $\left.-t \leq b_{i}<t-1\right\}$,

so Equation (4.4) is satisfied. As mentioned, it is desirable that $Y$ be small, i.e, that $\mathcal{T}$ be a short basis. While we cannot control $\mathcal{D}_{0}$, we have chosen the additional vectors $2 t \cdot e_{i}$ to be as short as possible, that is, just long enough to guarantee Equation (4.4).

We turn to the general case, where the basis $\mathcal{D}_{0}$ of $H$ might not consist of axis-parallel vectors. Again, we augment $\mathcal{D}_{0}$ with axis-parallel vectors to form a full-rank basis, namely, $\mathcal{T}=\mathcal{D}_{0} \cup\left\{2 t \cdot e_{i} \mid i \in I\right\}$ for some carefully chosen $I \subseteq[m]$, and define $\left(Y, y_{0}\right)$ and $f_{C}$ as above. However, it is now possible that an edge labeled $e_{j}(j \in[m] \backslash I)$ is not preserved by $f_{C}$. In order to analyze the behavior of the generator $e_{j}$, we apply a linear transformation that maps $\mathcal{T}$ to an axis-parallel basis. The image of $e_{j}$ under this transformation may be large in its $I$ coordinates, which means that many $e_{j}$-labeled edges are not preserved by $f_{C}$. In order to control the effect of these generators $e_{j}$, we need to choose $I$ so that the vectors $\left\{e_{i}\right\}_{i \in I}$ are nearly orthogonal to $\mathcal{D}_{0}$. Consequently, $\mathcal{T}$ is already close to being an orthogonal basis, thereby bounding the distortion of the linear transformation that "fixes" it to being orthogonal. We proceed to formally define these notions.

Given a finite ordered set of vectors $A \subseteq \mathbb{R}^{m}$, we write $\mathcal{M}_{A}$ for the matrix whose columns are the elements of $A$ in the standard basis. Note that if $A$ is a basis for $\mathbb{R}^{m}$ then $\mathcal{M}_{A}^{-1}$ is the change of basis matrix transforming a standardcoefficients vector to an $A$-coefficients vector (by multiplying column vectors of 


\section{ABELIAN GROUPS ARE POLYNOMIALLY STABLE}

coefficients from the left). Given a set $I \subseteq[m]$, let $\mathcal{M}_{A}^{I}$ denote the matrix $\mathcal{M}_{A}$ with the $i$-th row stricken out for every $i \in I$. Notice that if $|I|=m-\left|\mathcal{D}_{0}\right|$, then

$$
\left|\operatorname{det} \mathcal{M}_{\mathcal{D}_{0}}^{I}\right|=\left|\operatorname{det} \mathcal{M}_{\mathcal{D}_{0} \cup \mathcal{D}_{1}}\right|
$$

where $\mathcal{D}_{1}=\left\{e_{i} \mid i \in I\right\}$.

Definition 4.6. Let $\mathcal{D}_{0} \subseteq \mathbb{Z}^{m}$ be an ordered set of linearly independent vectors.

(i) Write $k=\left|\mathcal{D}_{0}\right|$. For a set of coordinates $I \subseteq[m]$ with $|I|=m-k$, the strength of $I$ with respect to $\mathcal{D}_{0}$ is $\left|\operatorname{det}\left(\mathcal{M}_{\mathcal{D}_{0}}^{I}\right)\right|$, that is, the unsigned volume of the parallelotope generated by $\mathcal{D}_{0} \cup\left\{e_{i}\right\}_{i \in I}$ (see Equation (4.5)).

(ii) We say that $I$, as above, is a set of strongest coordinates for $\mathcal{D}_{0}$ if $\left|\operatorname{det}\left(\mathcal{M}_{\mathcal{D}_{0}}^{I}\right)\right| \geq$ $\left|\operatorname{det}\left(\mathcal{M}_{\mathcal{D}_{0}}^{J}\right)\right|$ for every $J \subseteq[m]$ satisfying $|J|=m-k$. Note that in this case, $\left|\operatorname{det}\left(\mathcal{M}_{\mathcal{D}_{0}}^{I}\right)\right|$ is strictly positive.

(iii) For a set $\mathcal{D}_{1} \subseteq \mathbb{Z}^{m}$, we say the $\mathcal{D}_{1}$ is a standard complement for $\mathcal{D}_{0}$ if $\mathcal{D}_{0} \cup \mathcal{D}_{1}$ is a basis for $\mathbb{R}^{m}$ and $\mathcal{D}_{1}=\left\{e_{i}\right\}_{i \in I}$ for some $I \subseteq[m]$. A standard complement $\mathcal{D}_{1}$ for $\mathcal{D}_{0}$ as above is strong if $I$ is a set of strongest coordinates with respect to $\mathcal{D}_{0}$.

Note that for every set of strongest coordinates $I \subseteq[m]$ for $\mathcal{D}_{0}$, the set $\mathcal{D}_{1}=\left\{e_{i}\right\}_{i \in I}$ is a strong standard complement for $\mathcal{D}_{0}$. The following lemma formulates the near orthogonality condition mentioned above.

Lemma 4.7. Let $\mathcal{D}_{0} \subseteq \mathbb{Z}^{m}$ be a linearly independent set. Take a strong standard complement $\mathcal{D}_{1}$ for $\mathcal{D}_{0}$. Write $\mathcal{D}_{0}=\left\{h_{1}, \ldots, h_{k}\right\}$ and $\mathcal{D}_{1}=\left\{e_{i}\right\}_{i \in I}, I=$ $\left\{i_{1}, \ldots, i_{m-k}\right\}$, and let $\mathcal{D}=\left(h_{1}, \ldots, h_{k}, e_{i_{1}}, \ldots, e_{i_{m-k}}\right)$. Then, all entries of the lower $(m-k) \times m$ block of the matrix $\mathcal{M}_{\mathcal{D}}^{-1}$ are in the range $[-1,1]$

Proof. Permuting the rows of $\mathcal{M}_{\mathcal{D}}$ maintains the property that the right $m-k$ columns of $\mathcal{M}_{\mathcal{D}}$ are a strong standard complement for the left $k$ columns, and affects $\mathcal{M}_{\mathcal{D}}^{-1}$ by a permutation of its columns (in particular, it permutes the lower $(m-k) \times m$ block of $\left.\mathcal{M}_{\mathcal{D}}^{-1}\right)$. Therefore, we assume that $I=\{k+1, \ldots, m\}$ without loss of generality. Accordingly, $\mathcal{D}=\left(h_{1}, \ldots, h_{k}, e_{k+1}, \ldots, e_{m}\right)$. Let $k+1 \leq i \leq m$ and $1 \leq j \leq m$. Our task is to show that $\left|\left(\mathcal{M}_{\mathcal{D}}^{-1}\right)_{i, j}\right| \leq 1$. Note that the lower-right block of $\mathcal{M}_{\mathcal{D}}^{-1}$ is an $(m-k) \times(m-k)$ identity matrix. Thus, the claim holds whenever $j>k$. Henceforth, assume that $1 \leq j \leq k$. Let $\mathcal{M}_{\mathcal{D}}^{j, i}$ denote the matrix $\mathcal{M}_{\mathcal{D}}$ with the $j$-th row and $i$-th column removed. By Cramer's rule, the $(i, j)$-entry of $\mathcal{M}_{\mathcal{D}}^{-1}$ is either

$$
\frac{\operatorname{det} \mathcal{M}_{\mathcal{D}}^{j, i}}{\operatorname{det} \mathcal{M}_{\mathcal{D}}}
$$

or its negation. Hence, it suffices to show that $\left|\operatorname{det} \mathcal{M}_{\mathcal{D}}^{j, i}\right| \leq\left|\operatorname{det} \mathcal{M}_{\mathcal{D}}\right|$. By Equation $(4.5),\left|\operatorname{det} \mathcal{M}_{\mathcal{D}}\right|=\left|\operatorname{det} \mathcal{M}_{\mathcal{D}_{0}}^{I}\right|$. Similarly, $\left|\operatorname{det} \mathcal{M}_{\mathcal{D}}^{j, i}\right|=\left|\operatorname{det} \mathcal{M}_{\mathcal{D}_{0}}^{(I \backslash\{i\}) \cup\{j\}}\right|$. The claim follows since $I$ is a set of strongest coordinates for $\mathcal{D}_{0}$, and so $\left|\operatorname{det} \mathcal{M}_{\mathcal{D}_{0}}^{I}\right| \geq\left|\operatorname{det} \mathcal{M}_{\mathcal{D}_{0}}^{(I \backslash\{i\}) \cup\{j\}}\right|$. 


\section{OREN BECKER AND JONATHAN MOSHEIFF}

We need to fix, once and for all, a strong standard complement $\mathcal{C}\left(\mathcal{D}_{0}\right)$ for each linearly independent $\mathcal{D}_{0} \subseteq \mathbb{Z}^{m}$. For our purposes, it does not matter how this is done. However, for concreteness, we do it as follows:

Definition 4.8. For an ordered linearly independent subset $\mathcal{D}_{0} \subseteq \mathbb{Z}^{m}$, let $\mathcal{C}\left(\mathcal{D}_{0}\right) \subseteq \mathbb{Z}^{m}$ be the lexicographically least among all strong standard complements for $\mathcal{D}_{0}$. Let $I\left(\mathcal{D}_{0}\right)$ denote the subset of $[m]$ for which $\mathcal{C}\left(\mathcal{D}_{0}\right)=\left\{e_{i}\right\}_{i \in I\left(\mathcal{D}_{0}\right)}$.

Definition 4.9. Let $\mathcal{D}_{0} \subseteq \mathbb{Z}^{m}$ be a linearly independent set. Write $k=\left|\mathcal{D}_{0}\right|$, and order $I\left(\mathcal{D}_{0}\right)$ by writing $I\left(\mathcal{D}_{0}\right)=\left\{i_{k+1}, \ldots, i_{m}\right\}, i_{k+1}<\cdots<i_{m}$. Define an ordered basis $\mathcal{D}=\mathcal{D}_{0} \cup \mathcal{C}\left(\mathcal{D}_{0}\right)=\left(h_{1}, \ldots, h_{k}, e_{i_{k+1}}, \ldots, e_{i_{m}}\right)$ for $\mathbb{R}^{m}$. For $t \in \mathbb{N}$, define a discrete parallelotope

$$
P_{t}^{\mathcal{D}_{0}}=\left\{x \in \mathbb{Z}^{m} \mid \mathcal{M}_{\mathcal{D}}^{-1}(x) \in[0,1)^{k} \times[-t, t)^{m-k}\right\},
$$

and write $\hat{P}_{t}^{\mathcal{D}_{0}}$ for its canonical lift to $\mathbb{F}_{m}$ (see Section 4.2), to wit,

$$
\hat{P}_{t}^{\mathcal{D}_{0}}=\left\{\hat{x} \mid x \in P_{t}^{\mathcal{D}_{0}}\right\} .
$$

Lemma 4.10. Let $\mathcal{D}_{0} \subseteq \mathbb{Z}^{m}$ be a linearly independent set and $t \in \mathbb{N}$. Then,

(i) $P_{t}^{\mathcal{D}_{0}} \subseteq B_{\mathbb{Z}^{m}}\left(\left\|\mathcal{D}_{0}\right\|_{1}+\left(m-\left|\mathcal{D}_{0}\right|\right) \cdot t\right)$.

(ii) $\left|P_{t}^{\mathcal{D}_{0}}\right|=\left|P_{1}^{\mathcal{D}_{0}}\right| \cdot t^{m-\left|\mathcal{D}_{0}\right|}$.

(iii) Let $U=\mathbb{Z}^{m} /\left\langle\mathcal{D}_{0}\right\rangle$ and $u_{0} \in U$. Then, $\left(-P_{t}^{\mathcal{D}_{0}}+P_{t}^{\mathcal{D}_{0}}\right) \cdot u_{0} \subseteq P_{2 t}^{\mathcal{D}_{0}} \cdot u_{0}$.

Proof. The first statement follows from the triangle inequality of the $L^{1}$-norm in $\mathbb{Z}^{m}$. The second statement holds since $P_{t}^{\mathcal{D}_{0}}$ is the disjoint union of $t^{m-\left|\mathcal{D}_{0}\right|}$ translates of $P_{1}^{\mathcal{D}_{0}}$. We turn to prove the third statement.

Define $\mathcal{D}=\mathcal{D}_{0} \cup \mathcal{C}\left(\mathcal{D}_{0}\right)$ as in Definition 4.9. We have

$$
\begin{aligned}
\left(-P_{t}^{\mathcal{D}_{0}}+P_{t}^{\mathcal{D}_{0}}\right) \cdot u_{0} & =\left\{x \in \mathbb{Z}^{m} \mid \mathcal{M}_{\mathcal{D}}^{-1}(x) \in(-1,1)^{k} \times(-2 t, 2 t)^{m-k}\right\} \cdot u_{0} \\
& =\left\{x \in \mathbb{Z}^{m} \mid \mathcal{M}_{\mathcal{D}}^{-1}(x) \in[0,1)^{k} \times(-2 t, 2 t)^{m-k}\right\} \cdot u_{0} \\
& \subseteq P_{2 t}^{\mathcal{D}} \cdot u_{0}
\end{aligned}
$$

where the second equality above follows from the fact that $\mathcal{D}_{0} \subseteq \operatorname{Stab}_{\mathbb{Z}^{m}}\left(u_{0}\right)$.

The next proposition yields Tool C, namely, we show that for a pointed $\Gamma$-set $\left(V, v_{0}\right)$, there is an injection $f_{C}:\left(Y, y_{0}\right) \rightarrow\left(V, v_{0}\right)$, where $Y$ is a finite $\Gamma$-set and $\mathrm{Eq}\left(f_{C}\right)$ is large. We also provide some control over word-metric neighborhoods of $\operatorname{Im}\left(f_{C}\right)$ in $V$.

Proposition 4.11. Let $H \leq \mathbb{Z}^{m}, V=\mathbb{Z}^{m} / H, v_{0}=0+H$ and $t \in \mathbb{N}$. Take a basis $\mathcal{D}_{0}$ for $H$. Then, there is a finite quotient $Y$ of $V$, and an injective map $f_{C}: Y \rightarrow P_{t}^{\mathcal{D}_{0}} \cdot v_{0}$, such that: 


\section{ABELIAN GROUPS ARE POLYNOMIALLY STABLE}

(i) $\left|\mathrm{Eq}\left(f_{C}\right)\right| \geq\left(1-\frac{m-\left|\mathcal{D}_{0}\right|}{t}\right) \cdot|Y|$.

(ii) For every integer $\tilde{t} \geq 0,\left|B_{V}\left(\operatorname{Im}\left(f_{C}\right), \tilde{t}\right)\right| \leq\left(1+\frac{\tilde{t}}{t}\right)^{m-\left|\mathcal{D}_{0}\right|} \cdot\left|\operatorname{Im}\left(f_{C}\right)\right|$.

Proof. Let $k=\left|\mathcal{D}_{0}\right|$ and write $P_{r}=P_{r}^{\mathcal{D}_{0}}$ for every $r \in \mathbb{N}$. Let $\mathcal{D}=\mathcal{D}_{0} \cup \mathcal{C}\left(\mathcal{D}_{0}\right)$ and $\mathcal{T}=\mathcal{D}_{0} \cup 2 t \cdot \mathcal{C}\left(\mathcal{D}_{0}\right)$. Note that

$$
H=\left\langle\mathcal{D}_{0}\right\rangle=\left\{x \in \mathbb{Z}^{m} \mid \mathcal{M}_{\mathcal{D}}^{-1}(x) \in \mathbb{Z}^{k} \times\{0\}^{m-k}\right\}
$$

and

$$
\langle\mathcal{T}\rangle=\left\{x \in \mathbb{Z}^{m} \mid \mathcal{M}_{\mathcal{D}}^{-1}(x) \in \mathbb{Z}^{k} \times(2 t \mathbb{Z})^{m-k}\right\} .
$$

Define $Y=\mathbb{Z}^{m} /\langle\mathcal{T}\rangle$ and $y_{0}=0+\langle\mathcal{T}\rangle$. Let $f_{C}=F_{P_{t}, y_{0}, v_{0}}: P_{t} \cdot y_{0} \rightarrow$ $P_{t} \cdot v_{0}$. First, we show that $f_{C}$ is well-defined and injective. By the remark after Definition 3.8, it is enough to show that $\langle\mathcal{T}\rangle \cap\left(-P_{t}+P_{t}\right)=\left\langle\mathcal{D}_{0}\right\rangle \cap\left(-P_{t}+P_{t}\right)$. Clearly, the right-hand side is contained in the left-hand side, so we only need to show the reverse inclusion. Let $x \in-P_{t}+P_{t}$. Then, for $k+1 \leq i \leq m$, we have $\left(\mathcal{M}_{\mathcal{D}}^{-1}(x)\right)_{i} \in[-t, t)-[-t, t)=(-2 t, 2 t)$. If, further, $x \in\langle\mathcal{T}\rangle$, then $\left(\mathcal{M}_{\mathcal{D}}^{-1}(x)\right)_{i}=0$, and so $x \in\left\langle\mathcal{D}_{0}\right\rangle$, as required.

For $v \in V=\mathbb{Z}^{m} / H$ and $k+1 \leq i \leq m$, define $\left(\mathcal{M}_{\mathcal{D}}^{-1}(v)\right)_{i}=\left(\mathcal{M}_{\mathcal{D}}^{-1}(x)\right)_{i}$ using any $x \in \mathbb{Z}^{m}$ such that $v=x+H$. This is well-defined due to Equation (4.6). By Lemma 4.7, for every $k+1 \leq i \leq m$ and $1 \leq j \leq m$,

$$
\left|\left(\mathcal{M}_{\mathcal{D}}^{-1}\left(e_{j}+x\right)-\mathcal{M}_{\mathcal{D}}^{-1}(x)\right)_{i}\right| \leq 1 .
$$

We turn to proving (i) and (ii).

(i) First, we show that

$$
P_{t-1} \cdot y_{0} \subseteq \mathrm{Eq}\left(f_{C}\right)
$$

Write $S=\left\{e_{1}, \ldots, e_{m}\right\} \subseteq \mathbb{Z}^{m}$. Since the domain $P_{t} \cdot y_{0}$ of $f_{C}$ is equal to the entire set $Y$, all of its points are internal in $Y$. Therefore, a point $y \in P_{t-1} \cdot y_{0}$ belongs to $\mathrm{Eq}\left(f_{C}\right)$ if and only if $f_{C}$ preserves $y \stackrel{s}{\longrightarrow}$ for every $s \in S$. Hence, by Lemma 3.10, it is sufficient to prove that $\langle\mathcal{T}\rangle \cap$ $\left(-P_{t}+S+P_{t-1}\right)=\left\langle\mathcal{D}_{0}\right\rangle \cap\left(-P_{t}+S+P_{t-1}\right)$. Let $x \in-P_{t}+S+P_{t-1}$. By Equation $(4.8),\left(\mathcal{M}_{\mathcal{D}}^{-1}(x)\right)_{i} \in(-2 t, 2 t)$ for all $k+1 \leq i \leq m$. Using (4.6) and (4.7) as before, we see that $x \in\left\langle\mathcal{D}_{0}\right\rangle$ if and only if $x \in\langle\mathcal{T}\rangle$, which proves Inclusion (4.9). Finally, using Lemma 4.10(ii), we get

$$
\begin{aligned}
\left|\operatorname{Eq}\left(f_{C}\right)\right| & \geq\left|P_{t-1} \cdot y_{0}\right|=\left|P_{t-1}\right| \\
& =\left|P_{1}\right| \cdot(t-1)^{m-\left|\mathcal{D}_{0}\right|} \\
& =\left|P_{t}\right| \cdot\left(1-\frac{1}{t}\right)^{m-\left|\mathcal{D}_{0}\right|} \\
& \geq|Y| \cdot\left(1-\frac{m-\left|\mathcal{D}_{0}\right|}{t}\right) .
\end{aligned}
$$


(ii) Take an integer $\tilde{t} \geq 0$. First, we show that $B_{V}\left(P_{t} \cdot v_{0}, \tilde{t}\right) \subseteq P_{t+\tilde{t}} \cdot v_{0}$. Note that $P_{r} \cdot v_{0}=\left\{v \in V \mid\left(\mathcal{M}_{\mathcal{D}}^{-1}(v)\right)_{i} \in[-r, r)\right.$ for $\left.k+1 \leq i \leq m\right\}$ for every $r \in \mathbb{N}$. Let $v \in B_{V}\left(P_{t} \cdot v_{0}, \tilde{t}\right)$. By Equation $(4.8),\left(\mathcal{M}_{\mathcal{D}}^{-1}(v)\right)_{i} \in$ $[-(t+\tilde{t}), t+\tilde{t})$, so $v \in P_{t+\tilde{t}} \cdot v_{0}$. Finally, since $\operatorname{Im}\left(f_{C}\right)=P_{t} \cdot v_{0}$, using Lemma 4.10(ii) again, we get

$$
\begin{aligned}
\left|B_{V}\left(\operatorname{Im}\left(f_{C}\right), \tilde{t}\right)\right| & \leq\left|P_{t+\tilde{t}} \cdot v_{0}\right| \\
& =\left|P_{t}\right| \cdot\left(1+\frac{\tilde{t}}{t}\right)^{m-\left|\mathcal{D}_{0}\right|} \\
& =\left|\operatorname{Im}\left(f_{C}\right)\right| \cdot\left(1+\frac{\tilde{t}}{t}\right)^{m-\left|\mathcal{D}_{0}\right|} .
\end{aligned}
$$

\subsection{Tool B: The method of far-reaching short sets}

We turn to Tool $B$, in which we are given a pointed, usually infinite, $\Gamma$-set $\left(V, v_{0}\right)$ and a positive integer $t$. Our goal is to create a pointed $\Gamma$-set $\left(U, u_{0}\right)=$ $\left(\mathbb{Z}^{m} /\langle\mathcal{D}\rangle, 0+\langle\mathcal{D}\rangle\right)$ and a subgraph isomorphism $f_{B}:\left(P_{t}^{\mathcal{D}} \cdot u_{0}, u_{0}\right) \rightarrow\left(P_{t}^{\mathcal{D}} \cdot v_{0}, v_{0}\right)$. Here, $\mathcal{D} \subseteq \mathbb{Z}^{m}$ is a linearly independent set of our choice, and $P_{t}^{\mathcal{D}} \cdot v_{0}$ must be bounded within a ball of radius $O(t)$. By Lemma 4.10(i), a sufficient condition for this bound is that $\|\mathcal{D}\|_{1} \leq O(t)$. Throughout this section, the reader should keep the discussion in the beginning of Section 4 in mind, and, in particular, the realization of $\Gamma$ as the quotient $\mathbb{Z}^{m} / K$, and the constants $C_{d}$ and $t_{E}$.

As before, we may assume that $V$ is transitive, so it can be regarded as $\mathbb{Z}^{m} / H$ for some $K \leq H \leq \mathbb{Z}^{m}$, with $v_{0}=0+H$. Our strategy is to find a linearly independent set $\mathcal{D} \subseteq H$ of short vectors, such that the restriction $f_{B}$ of the quotient map $U=\mathbb{Z}^{m} /\langle\mathcal{D}\rangle \rightarrow \mathbb{Z}^{m} / H=V$ to $P_{t}^{\mathcal{D}} \cdot u_{0}$ is a subgraphisomorphism. To show that such a set $\mathcal{D}$ exists, we first prove Lemma 4.12, which asserts the existence of a subset $\mathcal{D} \subseteq H$, consisting of short vectors, such that all vectors in $H$ up to a certain length $R=R\left(\|\mathcal{D}\|_{1}, t\right)$ are spanned by $\mathcal{D}$. Proposition 4.13 then shows that this set $\mathcal{D}$ is suitable for our purposes.

Lemma 4.12. Let $K \leq H \leq \mathbb{Z}^{m}$ and $t_{E} \leq t \in \mathbb{N}$. Then, there is a linearly independent subset $\mathcal{D} \subseteq H$, such that $K \leq\langle\mathcal{D}\rangle$ and the following holds:

(i) $\|\mathcal{D}\|_{1} \leq 2 \cdot 7^{d} \cdot d^{2 d+2} \cdot t$,

(ii) $\langle\mathcal{D}\rangle \cap B_{\mathbb{Z}^{m}}(R)=H \cap B_{\mathbb{Z}^{m}}(R)$ for $R=2 \cdot\left(\|\mathcal{D}\|_{1}+d t\right)+1$.

Proof. Let $\tau: \mathbb{Z}^{m} \rightarrow \mathbb{Z}^{d} \oplus\{0\}^{m-d}$ denote the projection onto the first $d$ coordinates, and let Let $\bar{H}=\tau(H)$. For each $0 \leq i \leq d+1$, define $t_{i}=t \cdot\left(7 d^{2}\right)^{i}$. Consider the monotone nondecreasing sequence of integers

$$
\left\{\operatorname{rank}\left\langle\bar{H} \cap B_{\mathbb{Z}^{m}}\left(t_{i}\right)\right\rangle\right\}_{i=0}^{d+1} .
$$




\section{ABELIAN GROUPS ARE POLYNOMIALLY STABLE}

Since the last element is at most $d$, there must be an integer $0 \leq i \leq d$ such that

$$
\operatorname{rank}\left\langle\bar{H} \cap B_{\mathbb{Z}^{m}}\left(t_{i}\right)\right\rangle=\operatorname{rank}\left\langle\bar{H} \cap B_{\mathbb{Z}^{m}}\left(t_{i+1}\right)\right\rangle .
$$

Fix such an integer $i$, and define $G=\left\langle\bar{H} \cap B_{\mathbb{Z}^{m}}\left(t_{i+1}\right)\right\rangle$. In particular, $G \cap$ $B_{\mathbb{Z}^{m}}\left(t_{i}\right)=\bar{H} \cap B_{\mathbb{Z}^{m}}\left(t_{i}\right)$, and so,

$\operatorname{rank}\left\langle G \cap B_{\mathbb{Z}^{m}}\left(t_{i}\right)\right\rangle=\operatorname{rank}\left\langle\bar{H} \cap B_{\mathbb{Z}^{m}}\left(t_{i}\right)\right\rangle=\operatorname{rank}\left\langle\bar{H} \cap B_{\mathbb{Z}^{m}}\left(t_{i+1}\right)\right\rangle=\operatorname{rank} G$.

Hence, $\operatorname{rank}\left\langle G \cap B_{\mathbb{Z}^{m}}^{L^{2}}\left(t_{i}\right)\right\rangle=\operatorname{rank} G$, and so Proposition 4.5 yields a basis $\overline{\mathcal{D}}_{0}$ for $G$ satisfying

$$
\left\|\overline{\mathcal{D}}_{0}\right\|_{1} \leq d^{2} \cdot t_{i}
$$

Recall the definition of the set $T \subseteq \mathbb{Z}^{m}$ from the beginning of Section 4 . Construct a set $\mathcal{D}_{0} \subseteq H$ consisting of one preimage $h \in H$ for each $\bar{h} \in \overline{\mathcal{D}}_{0}$ (i.e., $\tau(h)=\bar{h}$ ), such that $h-\bar{h} \in T$ (this is possible since $K \leq H$ ). So, each of the last $m-d$ coordinates of each $h \in \mathcal{D}_{0}$ is in the range $0, \ldots, \beta_{m}-1$. Now, define $H_{T}=H \cap\left(\{0\}^{d} \oplus \mathbb{Z}^{m-d}\right)$. Then, $K \leq H_{T}$, and so $\operatorname{rank} H_{T}=m-d=$ $\operatorname{rank} K$. Since $K$ has a basis $\beta_{d+1} \cdot e_{d+1}, \ldots, \beta_{m} \cdot e_{m}$, which is contained in $B_{\{0\}^{d} \oplus \mathbb{Z}^{m-d}}^{L^{2}}\left(\beta_{E}\right)$, we conclude from Proposition 4.5 that $H_{T}$ has a basis $\mathcal{D}_{1}$, with $\left\|\mathcal{D}_{1}\right\|_{1} \leq(m-d)^{2} \cdot \beta_{E}$. Then, the set $\mathcal{D}=\mathcal{D}_{0} \cup \mathcal{D}_{1}$ is linearly independent, and

$$
\begin{aligned}
\|\mathcal{D}\|_{1} & =\left\|\mathcal{D}_{0}\right\|_{1}+\left\|\mathcal{D}_{1}\right\|_{1} \\
& \leq \sum_{x \in \overline{\mathcal{D}}_{0}}\left(\|x\|_{1}+(m-d) \cdot \beta_{E}\right)+(m-d)^{2} \cdot \beta_{E} \\
& =\left\|\overline{\mathcal{D}}_{0}\right\|_{1}+\left(\left|\overline{\mathcal{D}}_{0}\right| \cdot(m-d)+(m-d)^{2}\right) \cdot \beta_{E} \\
& \leq\left\|\overline{\mathcal{D}}_{0}\right\|_{1}+(d+(m-d)) \cdot(m-d) \cdot \beta_{E} \\
& \leq d^{2} \cdot t_{i}+m \cdot(m-d) \cdot \beta_{E}
\end{aligned}
$$

where the last inequality follows from Equation (4.10). Now, $t \geq t_{E}$ implies that $m \cdot(m-d) \cdot \beta_{E} \leq d \cdot t$, and so,

$$
\|\mathcal{D}\|_{1} \leq d^{2} \cdot t_{i}+d \cdot t \leq d^{2} \cdot\left(7 d^{2}\right)^{d} \cdot t+d \cdot t \leq 2 \cdot 7^{d} \cdot d^{2 d+2} \cdot t
$$

proving (i). Furthermore, for $R=2 \cdot\left(\|\mathcal{D}\|_{1}+d t\right)+1$,

$$
\begin{aligned}
R & \leq 2 \cdot\left(d^{2} \cdot t_{i}+m \cdot(m-d) \cdot \beta_{E}+d t\right)+1 \\
& \leq 2 \cdot\left(3 \cdot d^{2} \cdot t_{i}\right)+1 \leq 7 \cdot d^{2} \cdot t_{i}=t_{i+1} .
\end{aligned}
$$

Hence, to prove (ii) it suffices to show that

$$
\langle\mathcal{D}\rangle \cap B_{\mathbb{Z}^{m}}\left(t_{i+1}\right)=H \cap B_{\mathbb{Z}^{m}}\left(t_{i+1}\right) .
$$

Let $h \in H \cap B_{\mathbb{Z}^{m}}\left(t_{i+1}\right)$ and write $\bar{h}_{0}=\tau(h)$. So,

$$
\bar{h}_{0} \in \bar{H} \cap B_{\mathbb{Z}^{m}}\left(t_{i+1}\right) \subseteq G=\left\langle\overline{\mathcal{D}}_{0}\right\rangle .
$$

Hence, there exists $h_{0} \in\left\langle\mathcal{D}_{0}\right\rangle$ such that $\tau\left(h_{0}\right)=\bar{h}_{0}$, and so $h-h_{0} \in H_{T}=\left\langle\mathcal{D}_{1}\right\rangle$. Hence, $h=h_{0}+\left(h-h_{0}\right) \in\langle\mathcal{D}\rangle$, proving the claim. 
The following proposition yields Tool B.

Proposition 4.13. Let $K \leq H \leq \mathbb{Z}^{m}$ and $t_{E} \leq t \in \mathbb{N}$. Write $V=\mathbb{Z}^{m} / H$ and $v_{0}=0+H$. Then, there is a linearly independent set $\mathcal{D} \subseteq H, K \leq\langle\mathcal{D}\rangle$, satisfying the following:

(i) $P_{t}^{\mathcal{D}} \subseteq B_{\mathbb{Z}^{m}}\left(C_{d} \cdot t\right)$.

(ii) For $U=\mathbb{Z}^{m} /\langle\mathcal{D}\rangle$ and $u_{0}=0+\langle\mathcal{D}\rangle$, there is a subgraph isomorphism $f_{B}: P_{t}^{\mathcal{D}} \cdot u_{0} \rightarrow P_{t}^{\mathcal{D}} \cdot v_{0}$ (given by $f_{B}=F_{P_{t}^{\mathcal{D}}, u_{0}, v_{0}}$ ).

Proof. Apply Lemma 4.12 to $H$ and $t$ to obtain a linearly independent set $\mathcal{D}$ with $K \leq\langle\mathcal{D}\rangle$ such that

$$
\|\mathcal{D}\|_{1} \leq 2 \cdot 7^{d} \cdot d^{2 d+2} \cdot t
$$

and

$$
\langle\mathcal{D}\rangle \cap B_{\mathbb{Z}^{m}}(R)=H \cap B_{\mathbb{Z}^{m}}(R),
$$

where $R=2 \cdot\left(\|\mathcal{D}\|_{1}+d t\right)+1$. Write $P=P_{t}^{\mathcal{D}}$. The first claim follows from Lemma 4.10(i) since

$$
P \subseteq B_{\mathbb{Z}^{m}}\left(\|\mathcal{D}\|_{1}+(m-|\mathcal{D}|) \cdot t\right) \subseteq B_{\mathbb{Z}^{m}}\left(\|\mathcal{D}\|_{1}+d \cdot t\right)
$$

and

$$
\|\mathcal{D}\|_{1}+d \cdot t \leq 2 \cdot 7^{d} \cdot d^{2 d+2} \cdot t+d \cdot t \leq C_{d} \cdot t .
$$

We turn to proving that $F_{P_{t}^{\mathcal{D}}, u_{0}, v_{0}}$ is a subgraph isomorphism. By Lemma 3.11 , it suffices to show that, for $S_{1}=\left\{e_{1}, \ldots, e_{m}\right\} \cup\{0\} \subseteq \mathbb{Z}^{m}$,

$$
H \cap\left(-P+S_{1}+P\right)=\langle\mathcal{D}\rangle \cap\left(-P+S_{1}+P\right) .
$$

Equation (4.12) yields $-P+S_{1}+P \subseteq B_{\mathbb{Z}^{m}}\left(2 \cdot\left(\|\mathcal{D}\|_{1}+d t\right)+1\right)=B_{\mathbb{Z}^{m}}(R)$, hence the claim follows from Equation (4.11).

\subsection{Tool A: The bounded-addition method}

We turn to Tool $A$. Here, we are given a pointed $\mathbb{F}_{m}$-set $\left(X, x_{0}\right)$ and $t \in \mathbb{N}$ such that

$$
\operatorname{Box}_{\mathbb{F}_{m}}(t) \cdot x_{0} \subseteq X_{E},
$$

i.e., $X$ abides by the set of equations $E$ within a certain neighborhood of $x_{0}$. Our goal is to construct a pointed $\Gamma$-set $\left(U, u_{0}\right)$ and a subgraph isomorphism $f_{A}: B_{U}\left(u_{0}, r\right) \rightarrow B_{X}\left(x_{0}, r\right)$ for some $r \geq \Omega(t)$. A key notion in our proof is "bounded addition", formalized in the following definition:

Definition 4.14. Let $\mathcal{D} \subseteq B_{\mathbb{Z}^{m}}^{L^{\infty}}(R), R \in \mathbb{N}$. Define $[\mathcal{D}]_{R} \subseteq B_{\mathbb{Z}^{m}}^{L^{\infty}}(R)$ as the minimal subset of $\mathbb{Z}^{m}$ satisfying the following conditions:

(I) $\mathcal{D} \cup\{0\} \subset[\mathcal{D}]_{R}$. 


\section{ABELIAN GROUPS ARE POLYNOMIALLY STABLE}

(II) If $x \in[\mathcal{D}]_{R}$, then $-x \in[\mathcal{D}]_{R}$.

(III) If $x_{1}, x_{2} \in[\mathcal{D}]_{R}$ and $\left\|x_{1}+x_{2}\right\|_{\infty} \leq R$, then $x_{1}+x_{2} \in[\mathcal{D}]_{R}$.

Informally, the relevance of Definition 4.14 to the problem Tool A aims to solve is the following: Inclusion (4.13) yields a guarantee on the behavior of $X$ in a neighborhood of $x_{0}$ (see Lemma 4.20). Therefore, we would like to know which elements of $\mathbb{Z}^{m}$ can be generated from a given finite subset $\mathcal{D} \subseteq \mathbb{Z}^{m}$ without straying far from the origin.

We proceed to develop bounded addition in order to prove Lemma 4.19 below, and then use it to provide Tool A. We begin with two immediate observations about $[\mathcal{D}]_{R}$ without proof :

Lemma 4.15. Let $\mathcal{D} \subseteq B_{\mathbb{Z}^{m}}^{L^{\infty}}(R), R \in \mathbb{N}$. Then:

(i) A vector $y \in \mathbb{Z}^{m}$ belongs to $[\mathcal{D}]_{R}$ if and only if there is a sequence $\left\{y_{i}\right\}_{i=1}^{k} \subseteq$ $B_{\mathbb{Z}^{m}}^{L^{\infty}}(R)$ with $y=y_{k}$, in which every element $y_{i}$ satisfies at least one of the following:

(a) $y_{i} \in \mathcal{D}$, or

(b) $y_{i}$ is the negation of a previous element, or

(c) $y_{i}$ is the sum of two previous elements.

(ii) For $\mathcal{E} \subseteq \mathbb{Z}^{m}$, if $\mathcal{E} \subseteq[\mathcal{D}]_{R}$ then $[\mathcal{E}]_{R} \subseteq[\mathcal{D}]_{R}$.

The sequence $\left\{y_{i}\right\}_{i=1}^{k}$ in Lemma 4.15(i) is called a $(\mathcal{D}, R)$-generation-sequence for $y$.

Lemma 4.16 provides a technical result, which is then used by Lemmas $4.17,4.18$ and 4.19 to give sufficient conditions for membership in $[\mathcal{D}]_{R}$. The proof of Lemma 4.16 is inspired by Thomas Jefferson's method for proportional allocation of seats in the United States House of Representatives - a method used in many countries to this day. Recall that $\mathcal{D}^{ \pm}$is the set of elements of $\mathcal{D}$ and their negations.

Lemma 4.16. Let $\mathcal{D} \subseteq B_{\mathbb{Z}^{m}}^{L^{\infty}}(R), R \in \mathbb{N}$, and let $y \in\langle\mathcal{D}\rangle$. Then, there is a finite sequence $x_{1}, \ldots, x_{N}$ of elements of $\mathcal{D}^{ \pm}$, such that $y=\sum_{j=1}^{N} x_{j}$, and for every $0 \leq k \leq N$,

$$
\left\|\sum_{j=1}^{k} x_{j}-\frac{k}{N} \cdot y\right\|_{\infty} \leq 2 \cdot|\mathcal{D}| \cdot R .
$$

Proof. Let $M$ be a multiset of elements of $\mathcal{D}^{ \pm}$whose sum is $y$. Assume, without loss of generality, that $M$ does not contain both $x$ and $-x$ for any $x \in \mathcal{D}$. Write $\bar{x}_{1}, \ldots, \bar{x}_{p}(p \leq|\mathcal{D}|)$ for the distinct elements of $M$, and $a_{i}$ for the multiplicity of $\bar{x}_{i}$ in $M$. Thus, $y=\sum_{i=1}^{p} a_{i} \cdot \bar{x}_{i}$. Let $N=|M|=\sum_{i=1}^{p} a_{i}$. We seek to order the elements of $M$ in a sequence $x_{1}, \ldots, x_{N}$ satisfying the claim.

Let

$$
F=\left\{\left(N \cdot \frac{b}{a_{i}}, i\right) \in[0, N] \times[p] \mid i \in[p] \text { and } 1 \leq b \leq a_{i}\right\}
$$


It is helpful to think of an element $(z, i) \in F$ as a flag of color $i$, located at $z \in[0, N]$. So, for every $i \in[p]$, we have $a_{i}$ flags of color $i$, positioned evenly from $\frac{N}{a_{i}}$ to $N$. Let $\left(z_{1}, i_{1}\right), \ldots,\left(z_{N}, i_{N}\right)$ be a sequence, consisting of all elements of $F$, ordered so that $z_{1} \leq z_{2} \leq \cdots \leq z_{N}$. For $1 \leq j \leq N$, we set $x_{j}=\bar{x}_{i_{j}}$. Note that $y=\sum_{j=1}^{N} x_{j}$, so we only need to show that Equation (4.14) holds.

First, we would like to show that

$$
\forall j \in[N] \quad z_{j} \geq j .
$$

For $z \in[0, N]$, let $w_{z}=\left|\left\{j \in[N] \mid z_{j} \leq z\right\}\right|$, i.e., the number of flags up to location $z$. We claim that $w_{z} \leq z$. Indeed, $\mid\left\{j \in[N] \mid z_{j} \leq z\right.$ and $\left.i_{j}=i\right\} \mid=$ $\left\lfloor z \cdot \frac{a_{i}}{N}\right\rfloor$ for $i \in[p]$. Summing over $i$ yields

$$
w_{z}=\sum_{i=1}^{p}\left\lfloor z \cdot \frac{a_{i}}{N}\right\rfloor \leq \sum_{i=1}^{p} z \cdot \frac{a_{i}}{N}=z .
$$

In particular, taking $z=z_{j}$, we have $w_{z_{j}} \leq z_{j}$, but clearly $w_{z_{j}} \geq j$, and so Inequality (4.15) follows.

For $i \in[p]$ and $l \in[N]$, define $b_{i, l}=\left|\left\{1 \leq j \leq l \mid i_{j}=i\right\}\right|$, that is, the number of $i$-colored flags among the first $l$ flags. Let $k \in[N]$. We seek to bound the $L^{1}$-distance $\Delta$ between the vectors

$$
\left(b_{i, k}\right)_{i=1}^{p} \in \mathbb{R}^{p} \text { and }\left(\frac{k}{N} \cdot a_{i}\right)_{i=1}^{p} \in \mathbb{R}^{p} .
$$

Let $i \in[p]$. We would like to show that

$$
b_{i, k} \geq \frac{k}{N} \cdot a_{i}-1
$$

This clearly holds if $k<\frac{N}{a_{i}}$. Assume that $k \geq \frac{N}{a_{i}}$ and define $k^{\prime}=\left\lfloor\frac{k}{N} \cdot a_{i}\right\rfloor$. Let $j$ be minimal such that $b_{i, j}=k^{\prime}$. In other words, the $k^{\prime}$-th $i$-colored flag is $j$-th among all flags. Note that $z_{j}=\frac{N}{a_{i}} \cdot k^{\prime}$. By Inequality (4.15), $j \leq z_{j}=\frac{N}{a_{i}} \cdot k^{\prime} \leq k$. Equation (4.17) follows as

$$
b_{i, k} \geq b_{i, j}=k^{\prime} \geq \frac{k}{N} \cdot a_{i}-1 .
$$

Since the two vectors in (4.16) have the same sum $k$, the distance between them is

$$
\Delta=\sum_{i=1}^{p}\left|\frac{k}{N} \cdot a_{i}-b_{i, k}\right|=2 \cdot \sum_{i=1}^{p} \max \left\{0, \frac{k}{N} \cdot a_{i}-b_{i, k}\right\},
$$

so $\Delta \leq 2 p$ due to Equation (4.17). Now,

$$
\begin{aligned}
\left\|\sum_{j=1}^{k} x_{j}-\frac{k}{N} \cdot y\right\|_{\infty} & =\left\|\sum_{i=1}^{p} b_{i, k} \cdot x_{i}-\frac{k}{N} \cdot \sum_{i=1}^{p} a_{i} \cdot x_{i}\right\|_{\infty}=\left\|\sum_{i=1}^{p}\left(b_{i, k}-\frac{k}{N} \cdot a_{i}\right) \cdot x_{i}\right\|_{\infty} \\
& \leq \sum_{i=1}^{p}\left|b_{i, k}-\frac{k}{N} \cdot a_{i}\right| \cdot R \\
& =\Delta \cdot R \leq 2 p \cdot R \leq 2 \cdot|\mathcal{D}| \cdot R .
\end{aligned}
$$




\section{ABELIAN GROUPS ARE POLYNOMIALLY STABLE}

Lemma 4.17. Let $\mathcal{D} \subseteq B_{\mathbb{Z}^{m}}^{L^{\infty}}(R), R \in \mathbb{N}$, and $y \in\langle\mathcal{D}\rangle$. Then, $y \in[\mathcal{D}]_{2 \cdot|\mathcal{D}| \cdot R+\|y\|_{\infty}}$.

Proof. By Lemma 4.16, there is a sequence $x_{1}, \ldots, x_{m}$ of elements of $\mathcal{D}^{ \pm}$, such that $y=\sum_{j=1}^{m} x_{j}$, and for every $0 \leq k \leq m$, we have $\left\|\sum_{j=1}^{k} x_{j}-\frac{k}{m} \cdot y\right\|_{\infty} \leq$ $2 \cdot|\mathcal{D}| \cdot R$. Consequently, $\left\|\sum_{j=1}^{k} x_{j}\right\|_{\infty} \leq 2 \cdot|\mathcal{D}| \cdot R+\|y\|_{\infty}$, so by induction on $k$, each of the partial sums $\sum_{j=1}^{k} x_{j}$ belongs to $[\mathcal{D}]_{2 \cdot|\mathcal{D}| \cdot R+\|y\|_{\infty}}$. In particular, $k=m$ yields the claim.

Proposition 4.5 says that a lattice in $\mathbb{Z}^{m}$ which is generated by short elements has a short basis. The following lemma is an analogue statement in the context of bounded addition.

Lemma 4.18. Let $\mathcal{D} \subseteq B_{\mathbb{Z}^{m}}^{L^{\infty}}(R), R \in \mathbb{N}$. The lattice $\langle\mathcal{D}\rangle$ admits a basis contained in $[\mathcal{D}]_{5 m^{2} \cdot R} \cap B_{\mathbb{Z}^{m}}^{L^{\infty}}(m \cdot R)$.

Proof. Write $\mathcal{D}=\left\{x_{1}, \ldots, x_{p}\right\}$. For $0 \leq i \leq p$, let $\mathcal{D}_{i}=\left\{x_{1}, \ldots, x_{i}\right\}$. We prove by induction on $i$ that $\left\langle\mathcal{D}_{i}\right\rangle$ has a basis $\mathcal{E}_{i}$ contained in $[\mathcal{D}]_{5 m^{2} \cdot R} \cap B_{\mathbb{Z}^{m}}^{L^{\infty}}(m \cdot R)$. Taking $\mathcal{E}_{0}=\emptyset$, the base case $i=0$ is immediate. Assume that $i \geq 1$ and that $\left\langle\mathcal{D}_{i-1}\right\rangle$ has a basis $\mathcal{E}_{i-1}$ contained in $[\mathcal{D}]_{5 m^{2} \cdot R} \cap B_{\mathbb{Z}^{m}}^{L^{\infty}}(m \cdot R)$. We consider two generating sets for $\left\langle\mathcal{D}_{i}\right\rangle$ :

(I) The set $\mathcal{D}_{i}$, which is contained in $B_{\mathbb{Z}^{m}}^{L^{\infty}}(R)$.

(II) The set $\mathcal{E}_{i-1} \cup\left\{x_{i}\right\}$, which is contained in $B_{\mathbb{Z}^{m}}^{L^{\infty}}(m \cdot R)$, and has at most $m+1$ elements.

By virtue of the former generating set and by Proposition 4.5, $\left\langle\mathcal{D}_{i}\right\rangle$ has a basis $\mathcal{E}_{i} \subseteq B_{\mathbb{Z}^{m}}(m \cdot R) \subseteq B_{\mathbb{Z}^{m}}^{L^{\infty}}(m \cdot R)$. It is now enough to show that $\mathcal{E}_{i} \subseteq[\mathcal{D}]_{5 m^{2} \cdot R}$. Let $y \in \mathcal{E}_{i}$. Then, $\|y\|_{\infty} \leq m \cdot R$, and since $y \in \mathcal{E}_{i} \subseteq\left\langle\mathcal{D}_{i}\right\rangle$, we have $y \in$ $\left\langle\mathcal{E}_{i-1} \cup\left\{x_{i}\right\}\right\rangle$. As $\left\langle\mathcal{E}_{i} \cup\left\{x_{i}\right\}\right\rangle \subseteq B_{\mathbb{Z}^{m}}^{L^{\infty}}(m \cdot R)$ and $\left|\mathcal{E}_{i-1} \cup\left\{x_{i}\right\}\right| \leq m+1$, Lemma 4.17 implies that

$$
y \in\left[\mathcal{E}_{i-1} \cup\left\{x_{i}\right\}\right]_{2(m+1) \cdot m R+m R} \subseteq\left[\mathcal{E}_{i-1} \cup\left\{x_{i}\right\}\right]_{5 m^{2} \cdot R}
$$

As $\mathcal{E}_{i-1} \subseteq[\mathcal{D}]_{5 m^{2} \cdot R}$ by the induction hypothesis, and since $\left\{x_{i}\right\} \subseteq[\mathcal{D}]_{5 m^{2} \cdot R}$, it follows from Lemma 4.15 (ii) that $y \in[\mathcal{D}]_{5 m^{2} \cdot R}$.

Lemma 4.19. Let $\mathcal{D} \subseteq B_{\mathbb{Z}^{m}}^{L^{\infty}}(R), R \in \mathbb{N}$. Then, $\langle\mathcal{D}\rangle \cap B_{\mathbb{Z}^{m}}^{L^{\infty}}(R)=[\mathcal{D}]_{5 m^{2} \cdot R} \cap$ $B_{\mathbb{Z}^{m}}^{L^{\infty}}(R)$.

Proof. Given $x \in\langle\mathcal{D}\rangle \cap B_{\mathbb{Z}^{m}}^{L^{\infty}}(R)$, we need to show that $x \in[\mathcal{D}]_{5 m^{2} \cdot R}$. Lemma 4.18 yields a basis $\mathcal{E} \subseteq[\mathcal{D}]_{5 m^{2} \cdot R} \cap B_{\mathbb{Z}^{m}}^{L^{\infty}}(m \cdot R)$ for $\langle\mathcal{D}\rangle$. Since $x \in\langle\mathcal{E}\rangle$, Lemma 4.17 implies that $x \in[\mathcal{E}]_{2|\mathcal{E}| \cdot m R+R} \subseteq[\mathcal{E}]_{5 m^{2} \cdot R}$. Lemma 4.15(ii) yields the claim, since $\mathcal{E} \subseteq[\mathcal{D}]_{5 m^{2} \cdot R}$. 


\section{OREN BECKER AND JONATHAN MOSHEIFF}

At this point, we have established the required groundwork concerning bounded addition. Before proving our main proposition about Tool A, we also need the following lemma. It states that, within $X_{E}$, an $\mathbb{F}_{m}$-set $X$ behaves in a certain sense like a $\mathbb{Z}^{m}$-set. The reader should recall, from Section 4.2, the definition of a sorted word and the notation $\hat{w}$ for a given $w \in \mathbb{F}_{m}$.

Lemma 4.20. Let $X$ be an $\mathbb{F}_{m}$-set, $x \in X$ and $R \in \mathbb{N}$. Assume that $\operatorname{Box}_{\mathbb{F}_{m}}(R)$. $x \subseteq X_{E}$. Then, $w \cdot x=\hat{w} \cdot x$ for every $w \in B_{\mathbb{F}_{m}}(R)$.

Proof. First, we fix some notation: For a word $w \in \mathbb{F}_{m}$, whose reduced form is $w=\hat{e}_{i_{1}}^{\epsilon_{1}} \cdots \hat{e}_{i_{k}}^{\epsilon_{k}}, i_{1}, \ldots, i_{k} \in[m]$ and $\epsilon_{1}, \ldots, \epsilon_{k} \in\{+1,-1\}$, write $\iota(w)$ for the number of inversions in $w$, namely,

$$
\iota(w)=\mid\left\{\left(j_{1}, j_{2}\right) \mid 1 \leq j_{1}<j_{2} \leq k \text { and } i_{j_{1}}>i_{j_{2}}\right\} \mid .
$$

Let $w \in B_{\mathbb{F}_{m}}(R)$ and write $w=\hat{e}_{i_{1}}^{\epsilon_{1}} \cdots \hat{e}_{i_{k}}^{\epsilon_{k}}$ as above $(k \leq R)$. If $\iota(w)=0$, then $w$ is sorted, i.e., $w=\hat{w}$, and we are done. Otherwise, take the maximal $1 \leq l \leq k-1$ for which $i_{l}>i_{l+1}$. Let $w_{l+1}$ denote the suffix $\hat{e}_{i_{l+1}}^{\epsilon_{l+1}} \cdots \hat{e}_{i_{k}}^{\epsilon_{k}}$ of $w$. Then $w_{l+1}$ is a sorted word by the definition of $l$, and so $w_{l+1} \in \operatorname{Box}_{\mathbb{F}_{m}}(R)$, implying that $w_{l+1} \cdot x \in X_{E}$. Hence, $\left[\hat{e}_{i_{l}}^{-\epsilon_{l}}, \hat{e}_{i_{l+1}}^{\epsilon_{l+1}}\right] \cdot w_{l+1} \cdot x=w_{l+1} \cdot x$. Define $w^{\prime} \in \mathbb{F}_{m}$ by $w^{\prime}=\hat{e}_{i_{1}}^{\epsilon_{1}} \cdots \hat{e}_{i_{l}}^{\epsilon_{l}} \cdot\left[\hat{e}_{i_{l}}^{-\epsilon_{l}}, \hat{e}_{i_{l+1}}^{\epsilon_{l+1}}\right] \cdot w_{l+1}=\hat{e}_{i_{1}}^{\epsilon_{1}} \cdots \hat{e}_{i_{l-1}}^{\epsilon_{l-1}} \cdot \hat{e}_{i_{l+1}}^{\epsilon_{l+1}} \cdot \hat{e}_{i_{l}}^{\epsilon_{l}} \cdot \hat{e}_{i_{l+2}}^{\epsilon_{l+2}} \cdots \hat{e}_{k}^{\epsilon_{k}}$. Then, $\widehat{w^{\prime}}=\hat{w}, \iota\left(w^{\prime}\right)<\iota(w)$ and

$$
\begin{aligned}
w^{\prime} \cdot x & =\hat{e}_{i_{1}}^{\epsilon_{1}} \cdots \hat{e}_{i_{l}}^{\epsilon_{l}} \cdot\left(\left[\hat{e}_{i_{l}}^{-\epsilon_{l}}, \hat{e}_{i_{l+1}}^{\epsilon_{l+1}}\right] \cdot w_{l+1} \cdot x\right) \\
& =\hat{e}_{i_{1}}^{\epsilon_{1}} \cdots \hat{e}_{i_{l}}^{\epsilon_{l}} \cdot\left(w_{l+1} \cdot x\right) \\
& =w \cdot x .
\end{aligned}
$$

Therefore, the claim follows by induction.

We turn to our main statement in this section, which yields Tool A.

Proposition 4.21. Let $X$ be an $\mathbb{F}_{m}$-set, $x_{0} \in X$ and $r \in \mathbb{N}$. Assume that $\operatorname{Box}_{\mathbb{F}_{m}}\left(30 m^{3} \cdot r\right) \cdot x_{0} \subseteq X_{E}$. Let $H=\left\langle\pi\left(\operatorname{Stab}_{\mathbb{F}_{m}}\left(x_{0}\right) \cap B_{\mathbb{F}_{m}}(2 r+1)\right)\right\rangle \leq \mathbb{Z}^{m}$ and define the pointed $\mathbb{Z}^{m}$-set $\left(U, u_{0}\right)=\left(\mathbb{Z}^{m} / H, 0+H\right)$. Then, there is a subgraph isomorphism $f_{A}: B_{U}\left(u_{0}, r\right) \rightarrow B_{X}\left(x_{0}, r\right)$ (given by $\left.f_{A}=F_{B_{\mathbb{F}_{m}}}(r), u_{0}, x_{0}\right)$.

Proof. Write $R=2 r+1$, and note that

$$
\operatorname{Box}_{\mathbb{F}_{m}}\left(10 m^{3} \cdot R\right) \cdot x_{0} \subseteq X_{E} .
$$

Recall the natural surjection $\pi: \mathbb{F}_{m} \rightarrow \mathbb{Z}^{m}$, defined in the beginning of Section 4. Note that $\operatorname{Stab}_{\mathbb{F}_{m}}\left(u_{0}\right)=\pi^{-1}(H)$. Hence, by Lemma 3.12, it suffices to show that

$$
\operatorname{Stab}_{\mathbb{F}_{m}}\left(x_{0}\right) \cap B_{\mathbb{F}_{m}}(R)=\pi^{-1}(H) \cap B_{\mathbb{F}_{m}}(R) .
$$

The $\subseteq$ inclusion in Equation (4.18) is clear from the definition of $H$. We proceed to prove the $\supseteq$ inclusion. 


\section{ABELIAN GROUPS ARE POLYNOMIALLY STABLE}

Define $\mathcal{D}=\pi\left(\operatorname{Stab}_{\mathbb{F}_{m}}\left(x_{0}\right) \cap B_{\mathbb{F}_{m}}(R)\right)$, implying $H=\langle\mathcal{D}\rangle \leq \mathbb{Z}^{m}$. Note that

$$
\mathcal{D} \subseteq B_{\mathbb{Z}^{m}}^{L^{\infty}}(R)
$$

Let $w \in \pi^{-1}(H) \cap B_{\mathbb{F}_{m}}(R)$. It suffices to prove that $w \cdot x_{0}=x_{0}$. Now,

$$
\pi(w) \in H \cap B_{\mathbb{Z}^{m}}^{L^{\infty}}(R)=\langle\mathcal{D}\rangle \cap B_{\mathbb{Z}^{m}}^{L^{\infty}}(R) .
$$

By Lemma 4.19, Equations (4.19) and (4.20) imply that $\pi(w) \in[\mathcal{D}]_{5 m^{2} \cdot R}$. Let $v_{1}, \ldots, v_{k} \in B_{\mathbb{Z}^{m}}^{L^{\infty}}\left(5 m^{2} \cdot R\right)$ be a $\left(\mathcal{D}, 5 m^{2} \cdot R\right)$-generation-sequence for $\pi(w)$. In particular, $v_{k}=\pi(w)$. We shall construct, inductively, a sequence of sorted words $w_{1}, \ldots, w_{k} \in \mathbb{F}_{m}$, such that for each $1 \leq i \leq k$,

$$
w_{i} \in B_{\mathbb{F}_{m}}\left(5 m^{3} \cdot R\right), \quad \pi\left(w_{i}\right)=v_{i} \quad \text { and } \quad w_{i} \cdot x_{0}=x_{0} .
$$

Let $1 \leq i \leq k$, and assume that a sequence of sorted words $w_{1}, \ldots, w_{i-1} \in$ $\mathbb{F}_{m}$, satisfying $(4.21)$, has been constructed. We define $w_{i}$ by considering three separate cases:

(I) Assume that $v_{i} \in \mathcal{D}$. By the definition of $\mathcal{D}$, there is $u \in \operatorname{Stab}_{\mathbb{F}_{m}}\left(x_{0}\right) \cap$ $B_{\mathbb{F}_{m}}(R)$ satisfying $\pi(u)=v_{i}$. Since $\operatorname{Box}_{\mathbb{F}_{m}}(R) \cdot x_{0} \subseteq X_{E}$, Lemma 4.20 implies that $\hat{u} \cdot x_{0}=u \cdot x_{0}$. Define $w_{i}=\hat{u}$. Then, $w_{i} \cdot x_{0}=x_{0}$ since $u \in \operatorname{Stab}_{\mathbb{F}_{m}}\left(x_{0}\right)$, and $w_{i} \in B_{\mathbb{F}_{m}}(R)$ since $u \in B_{\mathbb{F}_{m}}(R)$.

(II) Otherwise, assume that there is $1 \leq j<i$ for which $v_{i}=-v_{j}$. By the induction hypothesis, $w_{j} \in B_{\mathbb{F}_{m}}\left(5 m^{3} \cdot R\right)$ and $w_{j} \cdot x_{0}=x_{0}$. So, the same holds for $w_{j}^{-1}$. Since $\operatorname{Box}_{\mathbb{F}_{m}}\left(5 m^{3} \cdot R\right) \cdot x_{0} \subseteq X_{E}$, Lemma 4.20 implies that $\widehat{w_{j}^{-1}} \cdot x_{0}=w_{j}^{-1} \cdot x_{0}=x_{0}$. Define $w_{i}=\widehat{w_{j}^{-1}}$.

(III) Otherwise, there must be integers $j_{1}, j_{2} \in[i-1]$ such that $v_{i}=v_{j_{1}}+v_{j_{2}}$. Define $u=w_{j_{1}} \cdot w_{j_{2}} \in B_{\mathbb{F}_{m}}\left(10 m^{3} \cdot R\right)$. Then,

$$
\|\pi(u)\|_{1}=\left\|v_{j_{1}}+v_{j_{2}}\right\|_{1}=\left\|v_{i}\right\|_{1} \leq m \cdot\left\|v_{i}\right\|_{\infty} \leq 5 m^{3} \cdot R .
$$

This means that, although the length of $u \in \mathbb{F}_{m}$ is merely bounded by $10 m^{3} \cdot R$, the length of the sorted word $\hat{u} \in \mathbb{F}_{m}$ is bounded by $5 m^{3} \cdot R$. Define $w_{i}=\hat{u}$. Since $\operatorname{Box}_{\mathbb{F}_{m}}\left(10 m^{3} \cdot R\right) \cdot x_{0} \subseteq X_{E}$, Lemma 4.20 implies that, $w_{i} \cdot x_{0}=u \cdot x_{0}=w_{j_{1}} \cdot w_{j_{2}} \cdot x_{0}=x_{0}$.

Finally, $\hat{w}=w_{k}$ since $w_{k}$ is sorted and $\pi\left(w_{k}\right)=v_{k}=\pi(w)$. As $w \in B_{\mathbb{F}_{m}}(R)$ and $\operatorname{Box}_{\mathbb{F}_{m}}(R) \cdot x_{0} \subseteq X_{E}$, Lemma 4.20 implies that $w \cdot x_{0}=\hat{w} \cdot x_{0}$. Thus, $w \cdot x_{0}=w_{k} \cdot x_{0}=x_{0}$, as claimed.

\subsection{The tiling algorithm - proof of the main theorem}

We can now implement the algorithm outlined in Section 4.1.1. The reader should recall the definitions and objects fixed in the beginning of Section 4. In particular we shall refer to the equation-set $E$, the constants $C_{d}$ and $t_{E}$, the integers $\left\{\beta_{i}\right\}_{i=d+1}^{m}$, the generators $\left\{\hat{e}_{1}, \ldots, \hat{e}_{m}\right\}$ of $\mathbb{F}_{m}$ and the set $\hat{T} \subseteq \mathbb{F}_{m}$. Given an $\mathbb{F}_{m}$-set $X$, we first discuss the injection of a single tile into $X$ by means of Tools A, B and C. 


\section{OREN BECKER AND JONATHAN MOSHEIFF}

Definition 4.22. Let $X$ be an $\mathbb{F}_{m}$-set, $x \in X$, and $t \in \mathbb{N}$.

(i) We say that $x$ admits a t-parallelotope if there is a linearly independent set $\mathcal{D} \subseteq \mathbb{Z}^{m}$, where $K \leq\langle\mathcal{D}\rangle$, such that the pointed subset $\left(P_{t}^{\mathcal{D}} \cdot u_{0}, u_{0}\right)$ of $\left(U, u_{0}\right)=\left(\mathbb{Z}^{m} /\langle\mathcal{D}\rangle, 0+\langle\mathcal{D}\rangle\right)$ is subgraph isomorphic to $\left(\hat{P}_{t}^{\mathcal{D}} \cdot x, x\right)$, and

$$
\hat{P}_{t}^{\mathcal{D}} \subseteq B_{\mathbb{F}_{m}}\left(C_{d} \cdot t\right)
$$

More elaborately, we also say that $x$ admits the t-parallelotope $P_{t}^{\mathcal{D}}$.

(ii) Fix some arbitrary well-ordering $\prec$ on finite subsets of $\mathbb{Z}^{m}$, e.g., let $\prec$ order $\mathbb{Z}^{m}$-subsets lexicographically with regard to some well-ordering on $\mathbb{Z}^{m}$ itself. If $x$ admits a $t$-parallelotope, we denote by $\mathcal{D}_{x, t}$ the $\prec$-minimal linearly independent set $\mathcal{D}$ for which $x$ admits the $t$-parallelotope $P_{t}^{\mathcal{D}}$.

Due to Equation (4.22), the collection of sets $\mathcal{D}$ satisfying condition $(i)$ above depends only on the ball of radius $C_{d} \cdot t$ centered at $x$. Since we take $D_{x, t}$ to be the $\prec$-minimum of this collection, the following simple fact follows.

Lemma 4.23. Let $(X, x)$ and $(\tilde{X}, \tilde{x})$ be pointed $\mathbb{F}_{m}$-sets. Let $t \in \mathbb{N}$, and assume that $B_{X}\left(x, C_{d} \cdot t\right)$ and $B_{\tilde{X}}\left(\tilde{x}, C_{d} \cdot t\right)$ are subgraph isomorphic as pointed sets, and that $x$ admits a t-parallelotope. Then, $\tilde{x}$ also admits a t-parallelotope and $\mathcal{D}_{x, t}=\mathcal{D}_{\tilde{x}, t}$.

In light of Definition 4.22, Proposition 4.13 can be rephrased as "For every $t \geq t_{E}$, every point in a $\Gamma$-set admits a $t$-parallelotope".

Definition 4.24. Let $X$ be an $\mathbb{F}_{m}$-set and $t \in \mathbb{N}$. A $t$-tile in $X$ is a pair $(x, f)$ such that:

(i) $x \in X$ admits a $2 t$-parallelotope, and

(ii) $f$ is a bijection from some finite $\Gamma$-set onto $\hat{P}_{t}^{\mathcal{D}} \cdot x \subseteq X$, where $\mathcal{D}=\mathcal{D}_{x, 2 t}$.

Note that, in the above definition we take $\mathcal{D}$ to be $\mathcal{D}_{x, 2 t}$, rather than $\mathcal{D}_{x, t}$. This "extra length" will be useful later in controlling the amount of interference between tiles.

As explained in Section 4.1.1, for a tile $(x, f)$, we want the set $\operatorname{Eq}(f)$ to be large. Also, we are interested in choosing, from among all tiles, a large collection of pairwise disjoint tiles. To this end, we seek to minimize the interference between tiles. The sets defined below are used to measure this interference:

Definition 4.25. Let $A \subseteq X$ for some $\mathbb{F}_{m}$-set $X$. For $t \in \mathbb{N}$, let

$\eta_{t}(A)=A \cup\{x \in X \mid$ There exists a $t$-tile $(x, f)$ such that $\operatorname{Im} f \cap A \neq \emptyset\}$.

We turn to prove the existence of tiles with good parameters (Proposition 4.27). We require the following observation. 


\section{ABELIAN GROUPS ARE POLYNOMIALLY STABLE}

Lemma 4.26. Let $X$ be an $\mathbb{F}_{m}$-set, $x \in X$ and $t \in \mathbb{N}$. Assume that

$$
\operatorname{Box}_{\mathbb{F}_{d}}(t) \cdot \hat{T} \cdot x \subseteq X_{E} .
$$

Then,

$$
\operatorname{Box}_{\mathbb{F}_{m}}(t) \subseteq X_{E}
$$

Proof. For $d+1 \leq i \leq m+1$, let

$$
\begin{aligned}
\hat{T}_{i} & =\left\{\prod_{j=i}^{m} \hat{e}_{j}^{\alpha_{j}} \mid 0 \leq \alpha_{j}<\beta_{j}\right\} \\
\hat{T}_{i}^{\infty} & =\left\{\prod_{j=i}^{m} \hat{e}_{j}^{\alpha_{j}} \mid \alpha_{j} \in \mathbb{Z}\right\}
\end{aligned}
$$

Clearly, $\hat{T}_{i} \cdot x \subseteq \hat{T}_{i}^{\infty} \cdot x$ for every $d+1 \leq i \leq m+1$. We show, by induction on $i=m+1, \ldots, d+1$, that this is in fact an equality. The lemma then follows since

$\operatorname{Box}_{\mathbb{F}_{m}}(t) \cdot x \subseteq \operatorname{Box}_{\mathbb{F}_{d}}(t) \cdot \hat{T}_{d+1}^{\infty} \cdot x=\operatorname{Box}_{\mathbb{F}_{d}}(t) \cdot \hat{T}_{d+1} \cdot x=\operatorname{Box}_{\mathbb{F}_{d}}(t) \cdot \hat{T} \cdot x$.

The base case $i=m+1$ is trivial, as $\hat{T}_{m+1}=\hat{T}_{m+1}^{\infty}=\left\{1_{\mathbb{F}_{m}}\right\}$. Let $d+1 \leq$ $i \leq m$ and assume that $\hat{T}_{i+1} \cdot x=\hat{T}_{i+1}^{\infty} \cdot x$. Let $w \in \hat{T}_{i}^{\infty}$. Note that we can write $w=\hat{e}_{i}^{\alpha_{i}} \cdot v$, where $\alpha_{i} \in \mathbb{Z}$ and $v \in \hat{T}_{i+1}^{\infty}$. By the induction hypothesis $v \cdot x \in \hat{T}_{i+1} \cdot x$. Since $\hat{T}_{i+1} \subseteq \hat{T}$, it follows from Equation (4.23) that $v \cdot x \in X_{E}$. In particular, $\hat{e}_{i}^{\beta_{i}} \cdot(v \cdot x)=v \cdot x$. Hence,

$$
w \cdot x=\hat{e}_{i}^{\alpha_{i}} \cdot(v \cdot x)=\hat{e}_{i}^{\bar{\alpha}_{i}} \cdot(v \cdot x) \in \hat{e}_{i}^{\bar{\alpha}_{i}} \cdot \hat{T}_{i+1} \cdot x
$$

where $0 \leq \bar{\alpha}_{i}<\beta_{i}$. As $\hat{e}_{i}^{\bar{\alpha}_{i}} \cdot \hat{T}_{i+1} \subseteq \hat{T}_{i}$, we have $w \cdot x \in \hat{T}_{i} \cdot x$.

Define the constant

$$
C_{\mathrm{Box}}=180 \cdot m^{3} \cdot C_{d} .
$$

Proposition 4.27. Let $t \geq t_{E}$ be an integer. Let $X$ be an $\mathbb{F}_{m}$-set, and $x \in X$ such that

$$
\operatorname{Box}_{\mathbb{F}_{d}}\left(C_{\mathrm{Box}} \cdot t\right) \cdot \hat{T} \cdot x \subseteq X_{E} .
$$

Then, there is a t-tile $(x, f)$ with

$$
|\mathrm{Eq}(f)| \geq\left(1-\frac{d}{t}\right) \cdot|\operatorname{Im} f|
$$

and for every $t_{E} \leq \tilde{t} \leq t$,

$$
\left|\eta_{\tilde{t}}(\operatorname{Im} f)\right| \leq\left(1+2 C_{d} \cdot \frac{\tilde{t}}{t}\right)^{d} \cdot|\operatorname{Im} f| .
$$

Furthermore,

$$
\left|\eta_{t}(\operatorname{Im} f)\right| \leq 2^{d} \cdot|\operatorname{Im} f|
$$




\section{OREN BECKER AND JONATHAN MOSHEIFF}

Proof. By Assumption (4.24) and Lemma 4.26, we have

$$
\operatorname{Box}_{\mathbb{F}_{m}}\left(C_{\mathrm{Box}} \cdot t\right) \cdot x \subseteq X_{E} .
$$

Define

$$
\begin{aligned}
H & =\left\langle\pi\left(\operatorname{Stab}_{\mathbb{F}_{m}}(x) \cap B_{\mathbb{F}_{m}}\left(12 \cdot C_{d} \cdot t+1\right)\right)\right\rangle, \text { and } \\
\left(U_{A}, u_{A}\right) & =\left(\mathbb{Z}^{m} / H, 0+H\right) .
\end{aligned}
$$

Note that $\left\{\hat{e}_{d+1}^{\beta_{d+1}}, \ldots, \hat{e}_{m}^{\beta_{m}}\right\} \subseteq \operatorname{Stab}_{\mathbb{F}_{m}}(x) \cap B_{\mathbb{F}_{m}}\left(12 \cdot C_{d} \cdot t+1\right)$, since $x \in X_{E}$ and $12 \cdot C_{d} \cdot t+1 \geq C_{d} \geq \beta_{E}$. Thus, $K \leq H$, so $U_{A}$ is a $\Gamma$-set. By virtue of Inclusion (4.28), Proposition 4.21 (Tool A) yields a subgraph isomorphism

$$
f_{A}: B_{U_{A}}\left(u_{A}, 6 C_{d} \cdot t\right) \rightarrow B_{X}\left(x, 6 C_{d} \cdot t\right) .
$$

By applying Proposition 4.13 (Tool B) to $H$ and $2 t$, it follows that $u_{A}$ admits the $2 t$-parallelotope $P_{2 t}^{\mathcal{D}}$ where $\mathcal{D}=\mathcal{D}_{u_{A}, 2 t}$ and $K \leq\langle\mathcal{D}\rangle$. Hence, for

$$
\left(U_{B}, u_{B}\right)=\left(\mathbb{Z}^{m} /\langle\mathcal{D}\rangle, 0+\langle\mathcal{D}\rangle\right)
$$

we have a subgraph isomorphism $f_{B}: P_{2 t}^{\mathcal{D}} \cdot u_{B} \rightarrow P_{2 t}^{\mathcal{D}} \cdot u_{A}$. Since $P_{2 t}^{\mathcal{D}} \cdot u_{A} \subseteq$ $B_{U_{A}}\left(u_{A}, C_{d} \cdot 2 t\right.$ ) (due to Equation (4.22)), we can define $f_{A B}: P_{2 t}^{\mathcal{D}} \cdot u_{B} \rightarrow \hat{P}_{2 t}^{\mathcal{D}} \cdot x$ by $f_{A B}=f_{A} \circ f_{B}$, and $f_{A B}$ is a subgraph isomorphism since both $f_{A}$ and $f_{B}$ are. In particular, $x$ admits a $2 t$-parallelotope. By virtue of the subgraph isomorphism $f_{A}$, the balls $B_{U_{A}}\left(u_{A}, C_{d} \cdot 2 t\right)$ and $B_{X}\left(x, C_{d} \cdot 2 t\right)$ are isomorphic, so Lemma 4.23 implies that $\mathcal{D}_{u_{A}, 2 t}=\mathcal{D}=\mathcal{D}_{x, 2 t}$.

We now apply Proposition 4.11 (Tool $\mathrm{C}$ ) to $\langle\mathcal{D}\rangle$ with the basis $\mathcal{D}$, and to the parameter $t$. This yields a finite $\Gamma$-set $Y$ and a bijection $f_{C}$ from $Y$ onto $P_{t}^{\mathcal{D}} \cdot u_{B}$, with

$$
\left|\mathrm{Eq}\left(f_{C}\right)\right| \geq\left(1-\frac{m-|\mathcal{D}|}{t}\right) \cdot|Y| \geq\left(1-\frac{d}{t}\right) \cdot|Y| .
$$

Note that the restriction of $f_{A B}$ to $\operatorname{Im} f_{C}=P_{t}^{\mathcal{D}} \cdot u_{B}$ is a subgraph isomorphism onto $\hat{P}_{t}^{\mathcal{D}} \cdot x$. Thus, $f=f_{A B} \circ f_{C}$ is a bijection from $Y$ onto $\hat{P}_{t}^{D} \cdot x$, so $(x, f)$ is a $t$-tile. Also, $\mathrm{Eq}(f)=\left|\mathrm{Eq}\left(f_{C}\right)\right| \geq\left(1-\frac{d}{t}\right) \cdot|\operatorname{Im} f|$, proving Equation (4.25). We turn to prove Equations (4.26) and (4.27).

Let $t_{E} \leq \tilde{t} \leq t$, and consider a $\tilde{t}$-tile $(\tilde{x}, \tilde{f})$ in $X$. Denote $\tilde{\mathcal{D}}=\mathcal{D}_{\tilde{x}, 2 \tilde{t}}$. Assume that $\operatorname{Im} \tilde{f} \cap \operatorname{Im} f \neq \emptyset$, i.e., $\hat{P}_{\tilde{t}}^{\tilde{\mathcal{D}}} \cdot x \cap \hat{P}_{t}^{\mathcal{D}} \cdot x \neq \emptyset$. Then, $\tilde{x} \in W \cdot x$, where

$$
W=\left(\hat{P}_{\tilde{t}}^{\tilde{D}}\right)^{-1} \cdot \hat{P}_{t}^{\mathcal{D}}
$$

In other words, $\eta_{\tilde{t}}(\operatorname{Im} f) \subseteq W \cdot x$. Since $W \subseteq\left(\hat{P}_{2 \tilde{\tilde{t}}}^{\tilde{\mathcal{D}}}\right)^{-1} \cdot \hat{P}_{2 t}^{\mathcal{D}}$, Equation $(4.22)$ implies that

$$
W \subseteq B_{\mathbb{F}_{m}}\left(2 C_{d} \cdot \tilde{t}+2 C_{d} \cdot t\right) \subseteq B_{\mathbb{F}_{m}}\left(4 C_{d} \cdot t\right) .
$$




\section{ABELIAN GROUPS ARE POLYNOMIALLY STABLE}

Hence, we may consider the restriction of $f_{A}: B_{U_{A}}\left(u_{A}, 6 C_{d} \cdot t\right) \rightarrow B_{X}\left(x, 6 C_{d} \cdot t\right)$ to $W \cdot u_{A}$, which is a subgraph isomorphism $W \cdot u_{A} \rightarrow W \cdot x$. Since $\left|\eta_{\tilde{t}}(\operatorname{Im} f)\right| \leq$ $|W \cdot x|$, it follows that

$$
\left|\eta_{\tilde{t}}(\operatorname{Im} f)\right| \leq\left|W \cdot u_{A}\right|
$$

Now,

$$
\begin{aligned}
\left|W \cdot u_{A}\right| & =\left|\left(P_{\tilde{t}}^{\tilde{\mathcal{D}}}\right)^{-1} \cdot P_{t}^{\mathcal{D}} \cdot u_{A}\right| \leq\left|\left(P_{2 \tilde{t}}^{\tilde{\mathcal{D}}}\right)^{-1} \cdot P_{t}^{\mathcal{D}} \cdot u_{A}\right| \\
& \leq\left|B_{U_{A}}\left(P_{t}^{\mathcal{D}} \cdot u_{A}, 2 C_{d} \cdot \tilde{t}\right)\right|=\left|B_{U_{B}}\left(P_{t}^{\mathcal{D}} \cdot u_{B}, 2 C_{d} \cdot \tilde{t}\right)\right| \\
& \leq\left(1+\frac{2 C_{d} \cdot \tilde{t}}{t}\right)^{d} \cdot\left|\operatorname{Im}\left(f_{C}\right)\right|
\end{aligned}
$$

where the second inequality follows from Equation (4.22), and the third from Proposition 4.11(ii). Equation (4.26) follows since $\left|\operatorname{Im}\left(f_{C}\right)\right|=|\operatorname{Im} f|$.

We turn to prove Equation (4.27). Observe that Equation (4.27) is a tighter version of (4.26) in the special case $\tilde{t}=t$. Hence, we continue with the existing notation, and assume further that $\tilde{t}=t$. Note that, since $\Gamma$ is abelian, all transitive $\Gamma$-sets are Cayley graphs of quotients of $\Gamma$, and so balls of the same radius in the $\Gamma$-set $U_{A}$ are isomorphic.

We have

$$
B_{X}\left(\tilde{x}, 2 C_{d} \cdot t\right) \subseteq B_{X}\left(W \cdot x, 2 C_{d} \cdot t\right) \subseteq B_{X}\left(x, 6 C_{d} \cdot t\right)=\operatorname{Im} f_{A}
$$

Thus, by virtue of the subgraph isomorphism $f_{A}$, the balls of radius $2 C_{d} \cdot t$ centered at $x$ and at $\tilde{x}$ are both isomorphic, as subgraphs of $X$, to balls of the same radius in the $\Gamma$-set $U_{A}$. Hence, these two balls are also subgraph isomorphic to each other. Consequently, $\mathcal{D}=\tilde{\mathcal{D}}$ due to Lemma 4.23 , and so $W=\left(\hat{P}_{t}^{\mathcal{D}}\right)^{-1} \cdot \hat{P}_{t}^{\mathcal{D}}$.

Now, Lemma 4.10(iii) implies that $W \cdot u_{B}=\left(\hat{P}_{t}^{\mathcal{D}}\right)^{-1} \cdot \hat{P}_{t}^{\mathcal{D}} \cdot u_{B} \subseteq \hat{P}_{2 t}^{\mathcal{D}} \cdot u_{B}$. Hence, the subgraph isomorphism $f_{A B}: P_{2 t}^{\mathcal{D}} \cdot u_{B} \rightarrow \hat{P}_{2 t}^{\mathcal{D}} \cdot x$ restricts to a subgraph isomorphism $W \cdot u_{B} \rightarrow W \cdot x$. Thus,

$$
\left|\eta_{t}(\operatorname{Im} f)\right| \leq|W \cdot x|=\left|W \cdot u_{B}\right| \leq\left|\hat{P}_{2 t}^{\mathcal{D}} \cdot u_{B}\right| \leq\left|P_{2 t}^{\mathcal{D}}\right| \leq 2^{m-|\mathcal{D}|} \cdot\left|P_{t}^{\mathcal{D}}\right|
$$

where the last inequality follows from Lemma 4.10(ii). As $|\mathcal{D}| \geq m-d$ and $|\operatorname{Im} f|=\left|P_{t}^{\mathcal{D}}\right|$, Equation (4.27) follows.

We turn to discuss an iteration of our algorithm. We require the following observation.

Lemma 4.28. Let $\mathcal{C}=\left(A_{i}\right)_{i \in I}$ be a finite collection of finite sets. Let $c>0$, and assume that for each $i \in I$, at most $c \cdot\left|A_{i}\right|$ sets $A_{j}(j \in I)$ intersect $A_{i}$ (including $A_{i}$ itself). Say that $J \subseteq I$ is intersection-free if $A_{j_{1}} \cap A_{j_{2}}=\emptyset$ for all distinct $j_{1}, j_{2} \in J$. Then, I has an intersection-free subset $J$ such that $\left|\bigcup_{j \in J} A_{j}\right| \geq \frac{|I|}{c}$. 
Proof. Let $J$ be a maximal intersection-free subset of $I$, and let $M=\bigcup_{j \in J} A_{j}$. Note that $M$ intersects at most $c \cdot|M|$ sets out of $\left(A_{i}\right)_{i \in I}$. By maximality, each of the $|I|$ elements of $\mathcal{C}$ intersects $M$, and so $|I| \leq c \cdot|M|$.

In the following proposition, which describes a single iteration of the tiling algorithm, we think of the set $A$ as the image of the tiles already injected into $X$ in previous iterations.

Proposition 4.29. Let $X$ be a finite $\mathbb{F}_{m}$-set, $A \subseteq X$ and $t_{E} \leq t \in \mathbb{N}$. Then, there is a finite $\Gamma$-set $Y$ and an injection $f: Y \rightarrow X \backslash A$, with the following properties:

(i) $|\operatorname{Im} f| \geq \frac{1}{2^{d}} \cdot\left(|X|-\left(3 C_{\mathrm{Box}} \cdot t\right)^{d} \cdot|\operatorname{Tor}(\Gamma)| \cdot\left|X \backslash X_{E}\right|-\left|\eta_{t}(A)\right|\right)$.

(ii) $|\operatorname{Eq} f| \geq\left(1-\frac{d}{t}\right) \cdot|\operatorname{Im} f|$.

(iii) $\left|\eta_{\tilde{t}}(\operatorname{Im} f)\right| \leq\left(1+2 C_{d} \cdot \frac{\tilde{t}}{t}\right)^{d} \cdot|\operatorname{Im} f|$ for every $t_{E} \leq \tilde{t} \leq t$.

Proof. Let

$$
M=\left(\operatorname{Box}_{\mathbb{F}_{d}}\left(C_{\mathrm{Box}} \cdot t\right) \cdot \hat{T}\right)^{-1} \cdot\left(X \backslash X_{E}\right)
$$

and $\tilde{X}=X \backslash\left(M \cup \eta_{t}(A)\right)$. Note that $\operatorname{Box}_{\mathbb{F}_{d}}\left(C_{\mathrm{Box}} \cdot t\right) \cdot \hat{T} \cdot \tilde{X} \subseteq X_{E}$, so Proposition 4.27, applied to each $x \in \tilde{X}$ separately, yields a set of $t$-tiles $\left(x, f_{x}\right)_{x \in \tilde{X}}$.

Let $x \in \tilde{X}$. By Equation (4.27), $\eta_{t}\left(\operatorname{Im} f_{x}\right) \leq 2^{d} \cdot\left|\operatorname{Im} f_{x}\right|$, so $\operatorname{Im} f_{x}$ intersects at most $2^{d} \cdot\left|\operatorname{Im} f_{x}\right|$ of the sets $\operatorname{Im} f_{x^{\prime}}\left(x^{\prime} \in \tilde{X}\right)$. In other words, the collection $\left(\operatorname{Im} f_{x}\right)_{x \in \tilde{X}}$ satisfies the requirements of Lemma 4.28, with $c=2^{d}$. Hence, there is a subset $J \subseteq \tilde{X}$ such that the sets $\left(\operatorname{Im} f_{x}\right)_{x \in J}$ are pairwise disjoint, and their union is of size at least $2^{-d} \cdot|\tilde{X}|$. We create a $\Gamma$-set $Y=\coprod_{x \in J}$ domain $\left(f_{x}\right)$ and an injection $f=\coprod_{x \in J} f_{x}: Y \rightarrow X$. By the definition of $\tilde{X}$, we have $\tilde{X} \cap \eta_{t}(A)=\emptyset$. This means that if $\left(x, f_{x}\right)$ is a $t$-tile and $x \in \tilde{X}$, then $\operatorname{Im} f_{x} \subseteq X \backslash A$. By the definition of $f$, it follows that $\operatorname{Im} f \subseteq X \backslash A$. We proceed to prove that $f$ has the stated properties. First,

$$
|\operatorname{Im} f| \geq 2^{-d} \cdot|\tilde{X}| \geq 2^{-d}\left(|X|-|M|-\left|\eta_{t}(A)\right|\right),
$$

and so Property (i) follows since

$$
\begin{aligned}
|M| & \leq\left|\operatorname{Box}_{\mathbb{F}_{d}}\left(C_{\mathrm{Box}} \cdot t\right) \cdot \hat{T}\right| \cdot\left|X \backslash X_{E}\right|=\left(2 C_{\mathrm{Box}} \cdot t+1\right)^{d} \cdot|\operatorname{Tor}(\Gamma)| \cdot\left|X \backslash X_{E}\right| \\
& \leq\left(3 C_{\mathrm{Box}} \cdot t\right)^{d} \cdot|\operatorname{Tor}(\Gamma)| \cdot\left|X \backslash X_{E}\right| .
\end{aligned}
$$

Property (ii) holds since

$$
|\operatorname{Eq} f| \geq \sum_{x \in J}\left|\operatorname{Eq} f_{x}\right| \geq \sum_{x \in J}\left(1-\frac{d}{t}\right) \cdot\left|\operatorname{Im} f_{x}\right|=\left(1-\frac{d}{t}\right) \cdot|\operatorname{Im} f|,
$$




\section{ABELIAN GROUPS ARE POLYNOMIALLY STABLE}

where the second inequality is guaranteed by Equation (4.25).

Property (iii) follows since, for $t_{E} \leq \tilde{t} \leq t$, Equation (4.26) yields

$\left|\eta_{\tilde{t}}(\operatorname{Im} f)\right| \leq \sum_{x \in J}\left|\eta_{\tilde{t}}\left(\operatorname{Im} f_{x}\right)\right| \leq \sum_{x \in J}\left(1+2 C_{d} \cdot \frac{\tilde{t}}{t}\right)^{d} \cdot\left|\operatorname{Im} f_{x}\right|=\left(1+2 C_{d} \cdot \frac{\tilde{t}}{t}\right)^{d} \cdot|\operatorname{Im} f|$

We turn to describe and analyze the tiling algorithm itself.

Proposition 4.30. Let $X$ be an $\mathbb{F}_{m}$-set, and denote $n=|X|$. Let $\delta>0$, and assume that $\frac{\left|X \backslash X_{E}\right|}{n} \leq \delta$. Then, there is a $\Gamma$-set $Y$ and an injection $f: Y \rightarrow X$, with

$$
|\mathrm{Eq}(f)| \geq\left(1-C \cdot \delta^{\frac{1}{Q}}\right) \cdot n,
$$

where $C>0$ is a constant which depends only on the equation-set $E$, and $Q \leq O\left(2^{d} \cdot d \cdot \max \left\{d \log d, \log \beta_{E}, 1\right\}\right)$ with an absolute implied constant.

Proof. We inductively define a sequence $f_{1}, \ldots, f_{s}$ of injections into $X$. The domain of each $f_{i}$ is a finite $\Gamma$-set.

Let $i \geq 1$. Assume that the injections $f_{1}, \ldots, f_{i-1}$ have already been defined. Let $A_{i}=\bigcup_{j=1}^{i-1} \operatorname{Im} f_{j}$, and define

$$
\begin{aligned}
h & =16 d \cdot C_{d}+1 \\
H_{i} & =24 C_{\mathrm{Box}} \cdot|\operatorname{Tor}(\Gamma)|^{\frac{1}{d}} \cdot \delta^{\frac{1}{d}} \cdot n \cdot h^{i-1} \\
b_{i} & =n-\left|A_{i}\right| \\
t_{i} & =\frac{b_{i}}{H_{i}} .
\end{aligned}
$$

It is helpful to remember that $H_{1}, H_{2}, \ldots$ is a geometric sequence. To generate the injection $f_{i}$, we apply Proposition 4.29 to the set $X$, with $t=\left\lfloor t_{i}\right\rfloor$ and $A=A_{i}$. We continue this process as long as $t_{i} \geq 2 t_{E}$ (hence, $\left\lfloor t_{i}\right\rfloor \geq t_{E}$ ), and denote the obtained injections by $f_{1}, \ldots, f_{s}$.

Proposition 4.29 guarantees that $\operatorname{Im} f_{i} \cap A_{i}=\emptyset$, so the sets $\left(\operatorname{Im} f_{i}\right)_{i=1}^{s}$ are pairwise disjoint. Hence, we may take $Y=\coprod_{i=1}^{s}$ domain $\left(f_{i}\right)$ and $f=\coprod_{i=1}^{s} f_{i}$, yielding an injection from the finite $\Gamma$-set $Y$ into $X$. We turn to prove that $f$ satisfies Equation (4.29).

Let $1 \leq j \leq s$. We seek to control the interference of an injection $f_{j}$ with subsequent iterations. Let $j<i \leq s$. By Proposition 4.29,

$$
\left|\eta_{\left\lfloor t_{i}\right\rfloor}\left(\operatorname{Im} f_{j}\right) \backslash \operatorname{Im} f_{j}\right| \leq\left(\left(1+\frac{2 C_{d} \cdot\left\lfloor t_{i}\right\rfloor}{\left\lfloor t_{j}\right\rfloor}\right)^{d}-1\right) \cdot\left|\operatorname{Im} f_{j}\right|
$$

Note that, as $t_{j} \geq t_{E} \geq 2$, we have $\left\lfloor t_{j}\right\rfloor \geq \frac{t_{j}}{2}$. Hence,

$$
2 \cdot \frac{C_{d} \cdot\left\lfloor t_{i}\right\rfloor}{\left\lfloor t_{j}\right\rfloor} \leq 4 \cdot \frac{C_{d} \cdot t_{i}}{t_{j}}=4 C_{d} \cdot \frac{b_{i} H_{j}}{b_{j} H_{i}} \leq 4 C_{d} \cdot \frac{H_{j}}{H_{i}} \leq 4 C_{d} \cdot \frac{1}{h} \leq \frac{1}{4 d} .
$$


In general, $(1+x)^{d} \leq 1+2 d \cdot x$ for $0 \leq x \leq \frac{1}{2 d}$, and so,

$$
\begin{aligned}
\left|\eta_{\left\lfloor t_{i}\right\rfloor}\left(\operatorname{Im} f_{j}\right) \backslash \operatorname{Im} f_{j}\right| & \leq \frac{4 d \cdot C_{d} \cdot\left\lfloor t_{i}\right\rfloor}{\left\lfloor t_{j}\right\rfloor} \cdot\left|\operatorname{Im} f_{j}\right| \leq \frac{8 d \cdot C_{d} \cdot t_{i}}{t_{j}} \cdot\left|\operatorname{Im} f_{j}\right| \\
& \leq \frac{8 d \cdot C_{d} \cdot t_{i}}{t_{j}} \cdot b_{j}=8 d \cdot C_{d} \cdot t_{i} \cdot H_{j} .
\end{aligned}
$$

We next give a lower bound on the number of points covered in the $i$-th iteration. For $1 \leq i \leq s$, Equation (4.30) yields

$$
\begin{aligned}
\left|\eta_{\left\lfloor t_{i}\right\rfloor}\left(A_{i}\right) \backslash A_{i}\right| & \leq \sum_{j=1}^{i-1}\left|\eta_{\left\lfloor t_{i}\right\rfloor}\left(\operatorname{Im} f_{j}\right) \backslash \operatorname{Im} f_{j}\right| \leq 8 d \cdot C_{d} \cdot t_{i} \cdot \sum_{j=1}^{i-1} H_{j} \\
& =8 d \cdot C_{d} \cdot t_{i} \cdot \frac{H_{i}-H_{1}}{h-1} \leq \frac{8 d \cdot C_{d}}{h-1} \cdot t_{i} \cdot H_{i} \leq \frac{t_{i} \cdot H_{i}}{2}=\frac{b_{i}}{2} .
\end{aligned}
$$

By Proposition 4.29(i),

$$
\left|\operatorname{Im} f_{i}\right| \geq 2^{-d} \cdot\left(n-\left(3 C_{\mathrm{Box}} \cdot\left\lfloor t_{i}\right\rfloor\right)^{d} \cdot|\operatorname{Tor}(\Gamma)| \cdot \delta n-\left|\eta_{\left\lfloor t_{i}\right\rfloor}\left(A_{i}\right)\right|\right) .
$$

Equation (4.31) yields

$$
\begin{aligned}
\left|\operatorname{Im} f_{i}\right| & \geq 2^{-d} \cdot\left(n-\left(3 C_{\mathrm{Box}} \cdot\left\lfloor t_{i}\right\rfloor\right)^{d} \cdot|\operatorname{Tor}(\Gamma)| \cdot \delta n-\frac{b_{i}}{2}-\left|A_{i}\right|\right) \\
& =2^{-d} \cdot\left(b_{i}-\left(3 C_{\mathrm{Box}} \cdot\left\lfloor t_{i}\right\rfloor\right)^{d} \cdot|\operatorname{Tor}(\Gamma)| \cdot \delta n-\frac{b_{i}}{2}\right) \\
& =2^{-d} \cdot\left(\frac{b_{i}}{2}-\left(3 C_{\mathrm{Box}} \cdot\left\lfloor t_{i}\right\rfloor\right)^{d} \cdot|\operatorname{Tor}(\Gamma)| \cdot \delta n\right) \\
& \geq 2^{-d} \cdot\left(\frac{b_{i}}{2}-\left(3 C_{\mathrm{Box}} \cdot t_{i}\right)^{d} \cdot|\operatorname{Tor}(\Gamma)| \cdot \delta n\right) .
\end{aligned}
$$

Now,

$$
\begin{aligned}
\left(3 C_{\mathrm{Box}} \cdot t_{i}\right)^{d} \cdot|\operatorname{Tor}(\Gamma)| \cdot \delta n & =\left(\frac{3 C_{\mathrm{Box}} \cdot b_{i}}{H_{i}}\right)^{d} \cdot|\operatorname{Tor}(\Gamma)| \cdot \delta n \\
& \leq\left(\frac{3 C_{\mathrm{Box}} \cdot b_{i}}{H_{1}}\right)^{d} \cdot|\operatorname{Tor}(\Gamma)| \cdot \delta n \\
& =\left(\frac{b_{i}}{8 n}\right)^{d} \cdot n \leq \frac{b_{i}}{8 n} \cdot n=\frac{b_{i}}{8} \leq \frac{b_{i}}{4}
\end{aligned}
$$

and consequently,

$$
\left|\operatorname{Im} f_{i}\right| \geq 2^{-(d+2)} \cdot b_{i} .
$$

Let $\gamma=1-2^{-(d+2)}$. Then, $b_{i}=b_{i-1}-\left|\operatorname{Im} f_{i-1}\right| \leq b_{i-1} \cdot \gamma$ for every $2 \leq i \leq s$, so $b_{i} \leq n \cdot \gamma^{i-1}$. 


\section{ABELIAN GROUPS ARE POLYNOMIALLY STABLE}

Finally, we turn to bound $|\mathrm{Eq} f|$ from below. Proposition 4.29(ii) gives

$$
\begin{aligned}
|\operatorname{Im} f|-|\operatorname{Eq} f| & =\left(\sum_{i=1}^{s}\left|\operatorname{Im} f_{i}\right|\right)-|\operatorname{Eq} f| \leq \sum_{i=1}^{s}\left(\left|\operatorname{Im} f_{i}\right|-\left|\operatorname{Eq} f_{i}\right|\right) \\
& \leq \sum_{i=1}^{s} \frac{d}{\left\lfloor t_{i}\right\rfloor} \cdot\left|\operatorname{Im} f_{i}\right| \leq \sum_{i=1}^{s} \frac{2 d}{t_{i}} \cdot\left|\operatorname{Im} f_{i}\right|=\sum_{i=1}^{s} 2 d \cdot \frac{\left|\operatorname{Im} f_{i}\right|}{b_{i}} \cdot H_{i} \\
& \leq \sum_{i=1}^{s} 2 d \cdot H_{i} \leq 2 d \cdot H_{s+1} .
\end{aligned}
$$

By the termination condition of the algorithm $t_{s+1} \leq 2 t_{E}$, but $t_{s+1}=\frac{b_{s+1}}{H_{s+1}}$ and $b_{s+1}=n-|\operatorname{Im} f|$. Consequently, $|\operatorname{Im} f| \geq n-2 t_{E} \cdot H_{s+1}$. Together with Equation (4.32), this implies

$$
|\operatorname{Eq} f|=|\operatorname{Im} f|-|\operatorname{Im} f \backslash \operatorname{Eq} f| \geq n-2\left(t_{E}+d\right) \cdot H_{s+1} .
$$

In order to bound $H_{s+1}$, we first need to bound $s$. Appealing again to the termination condition, we have

so

$$
2 t_{E} \leq t_{s}=\frac{b_{s}}{H_{s}} \leq \frac{n \cdot \gamma^{s-1}}{H_{1} \cdot h^{s-1}}
$$

$$
s \leq \log _{\gamma / h}\left(\frac{2 t_{E} \cdot H_{1}}{n}\right)+1 \leq \frac{\log \left(48 t_{E} \cdot C_{\mathrm{Box}} \cdot|\operatorname{Tor}(\Gamma)|^{\frac{1}{d}} \cdot \delta^{\frac{1}{d}}\right)}{\log \gamma-\log h}+1 .
$$

Hence,

$$
h^{s} \leq C^{\prime} \cdot \delta^{\frac{\log h}{d(\log \gamma-\log h)}}
$$

for some $C^{\prime}>0$ which depends only on the equation-set $E$. Thus,

$$
H_{s+1}=24 C_{\mathrm{Box}} \cdot|\operatorname{Tor}(\Gamma)|^{\frac{1}{d}} \cdot n \cdot \delta^{\frac{1}{d}} \cdot h^{s} \leq C^{\prime \prime} \cdot n \cdot \delta^{\frac{1}{d}+\frac{\log h}{d(\log \gamma-\log h)}}=C^{\prime \prime} \cdot n \cdot \delta^{\frac{1}{Q}} .
$$

where $C^{\prime \prime}=24 C_{\mathrm{Box}} \cdot|\operatorname{Tor}(\Gamma)|^{\frac{1}{d}} \cdot C^{\prime}$ and $Q=d \cdot\left(1-\frac{\log h}{\log \gamma}\right)$. Now,

$$
\begin{aligned}
Q & =d \cdot\left(1-\frac{\log h}{\log \gamma}\right) \leq d \cdot\left(1+\frac{\log h}{2^{-(d+2)}}\right) \leq O\left(2^{d} \cdot d \cdot \log \left(d \cdot C_{d}\right)\right) \\
& \leq O\left(2^{d} \cdot d \cdot \max \left\{d \log d, \log \beta_{E}, 1\right\}\right),
\end{aligned}
$$

where the implied constant is absolute. Therefore, the proposition follows from Equations (4.33) and (4.34).

Finally, we turn to proving the main theorem.

Proof of Theorem 1.16. By Lemma 3.15 and Proposition 4.30, for any finite $\Gamma$-set $X$ we have

$$
G_{E}(X) \leq|S| \cdot C \delta^{\frac{1}{Q}}
$$

where $C$ and $Q$ are as in Proposition 4.30. This yields Equation (4.2) from the beginning of Section 4, which is just a more elaborate form of Theorem 1.16. 


\section{A lower bound on the polynomial stability de- gree of $\mathbb{Z}^{d}$}

We turn to prove Theorem 1.17. We rely here on the formulation of stability in terms of group actions and labeled graphs, which was introduced in Section 3. The current section is independent of Section 4, except for the notion of a sorted word and the corresponding notation $\hat{r}$ for $r \in \mathbb{Z}^{d}$ (see Section 4.2).

Fix a constant $d \in \mathbb{N}$ and fix generator sets $S=\left\{e_{1}, \ldots, e_{d}\right\}$ and $\hat{S}=$ $\left\{\hat{e}_{1}, \ldots, \hat{e}_{d}\right\}$ for $\mathbb{Z}^{d}$ and $\mathbb{F}_{d}$, respectively. Note that we have a natural homomorphism $\pi: \mathbb{F}_{d} \rightarrow \mathbb{Z}^{d}$ which maps $\hat{e}_{i}$ to $e_{i}$. Let $E$ denote the commutator equation-set $E_{\text {comm }}^{d}=\left\{\hat{e}_{i} \hat{e}_{j} \hat{e}_{i}^{-1} \hat{e}_{j}^{-1} \mid 1 \leq i<j \leq d\right\}$ from Equation (1.2) and note that $\mathbb{F}_{d} /\left\langle\left\langle E_{\text {comm }}^{d}\right\rangle\right\rangle \cong \mathbb{Z}^{d}$. We seek to construct an infinite sequence of $\mathbb{F}_{d}$-sets $\left\{X_{t}\right\}_{t=1}^{\infty}$, with $\lim _{t \rightarrow \infty} L_{E}\left(X_{t}\right)=0$ and

$$
G_{E}\left(X_{t}\right) \geq \Omega_{t \rightarrow \infty}\left(L_{E}\left(X_{t}\right)^{\frac{1}{d}}\right) .
$$

By virtue of Proposition 3.5, Inequality (5.1) implies that $\operatorname{deg}\left(\mathrm{SR}_{E}\right) \geq d$, yielding Theorem 1.17.

Fix a positive integer $t$. Given $x \in \mathbb{Z}^{d}$, let $[x]$ denote its coset in the quotient group $\mathbb{Z}^{d} /\left(t \cdot \mathbb{Z}^{d}\right)$. We build the set $X_{t}$ by taking the natural action of $\mathbb{F}_{d}$ on $\mathbb{Z}^{d} /\left(t \cdot \mathbb{Z}^{d}\right)$, removing a single point, and slightly fixing the result so that it remains an $\mathbb{F}_{d^{-}}$set. Concretely, the ground-set for $X_{t}$ is $\left(\mathbb{Z}^{d} /\left(t \cdot \mathbb{Z}^{d}\right)\right) \backslash\{[0]\}$. Each generator $\hat{e}_{i}$ acts by taking $\left[(t-1) \cdot e_{i}\right]$ to $\left[e_{i}\right]$, and mapping any other $\left[\sum_{j=1}^{d} a_{j} \cdot e_{j}\right]$ to $\left[\left(\sum_{j=1}^{d} a_{j} \cdot e_{j}\right)+e_{i}\right]$, as usual.

We wish to compute $L_{E}\left(X_{t}\right)$. Let $1 \leq i<j \leq d$, and consider the set of points in which the relation $\left[\hat{e}_{i}, \hat{e}_{j}\right]$ is violated, namely $\left\{x \in X_{t} \mid \hat{e}_{i} \hat{e}_{j} \hat{e}_{i}^{-1} \hat{e}_{j}^{-1} \cdot x \neq x\right\}$. It is not hard to verify that these are exactly the three points

$$
\left\{\left[e_{i}\right],\left[e_{j}\right],\left[e_{i}+e_{j}\right]\right\} .
$$

Summing over the $\left(\begin{array}{l}d \\ 2\end{array}\right)$ elements of $E$, Definition 3.1 gives

$$
L_{E}\left(X_{t}\right)=\frac{1}{\left|X_{t}\right|} \cdot\left(\begin{array}{l}
d \\
2
\end{array}\right) \cdot 3
$$

Since $\left|X_{t}\right|=t^{d}-1$, it follows that $L_{E}\left(X_{t}\right) \leq O_{t \rightarrow \infty}\left(t^{-d}\right)$. Hence, the following proposition implies Equation (5.1), which yields Theorem 1.17.

Proposition 5.1. $G_{E}\left(X_{t}\right) \geq \Omega_{t \rightarrow \infty}\left(t^{-1}\right)$

Proof. Let $Y$ be a $\mathbb{Z}^{d}$-set of size $t^{d}-1$ and let $f: Y \rightarrow X_{t}$ be a bijection. We need to prove that $\|f\|_{S} \geq \Omega_{t \rightarrow \infty}\left(\frac{1}{t}\right)$.

Define the set

$$
X^{0}=X_{t} \backslash\left(\bigcup_{i=1}^{d}\left\{\left[k \cdot e_{i}\right] \mid 0 \leq k \leq t-1\right\}\right),
$$




\section{ABELIAN GROUPS ARE POLYNOMIALLY STABLE}

and let $Y^{0}=f^{-1}\left(X^{0}\right)$.

Let $U$ be a connected component of $Y$, i.e., an $\mathbb{F}_{d}$-orbit, and let $u \in U$. Note that the subset $A=\left\{\sum_{i=1}^{d} \alpha_{i} \cdot e_{i} \mid 0 \leq \alpha_{i} \leq t-1\right.$ for every $\left.1 \leq i \leq d\right\}$ of $\mathbb{Z}^{d}$ does not inject into $U$ at $u$, since $|A|=t^{d}>|Y| \geq|U|$. Hence, there are $p \neq q \in A$ such that $p \cdot u=q \cdot u$. Let $0 \neq r=p-q$, so $r \in \operatorname{Stab}_{\mathbb{Z}^{d}}(u)$. Hence, $r \in \operatorname{Stab}_{\mathbb{Z}^{d}}(y)$ for every $y \in U$. Write $r=\sum_{i=1}^{d} \alpha_{i} \cdot e_{i}$ where $-(t-1) \leq \alpha_{i} \leq t-1$ and define $w \in \mathbb{F}_{d}$ by $w=\hat{r}$ (see Section 4.2). We also write $w=w_{1} \cdots w_{k}$ as a reduced word, with $w_{i} \in \hat{S}^{ \pm}$. Note that $w \cdot y=y$ for every $y \in U$. However, it is not hard to see that $w \cdot x \neq x$ for each $x \in X^{0}$. Indeed, a non-trivial sorted word in $\operatorname{Stab}_{\mathbb{F}_{d}}(x)$ must contain some generator or its inverse at least $t$ times.

For $y \in U \cap Y^{0}$, let $P_{y}$ denote the set of edges in the path that starts at $y$ and proceeds as directed by $w$. Namely, the first such edge is $y \stackrel{w_{k}}{\longrightarrow} w_{k} \cdot y$, then $w_{k} \cdot y \stackrel{w_{k-1}}{\longrightarrow} w_{k-1} w_{k} \cdot y$, and so on (if $w_{i}$ is the inverse of a generator, we walk along an edge backwards). Assume that $f$ preserves all of the edges in $P_{y}$. Then $f(w \cdot y)=w \cdot f(y)$, but this is absurd, since $w \cdot y=y$, while $w \cdot f(y) \neq f(y)$, as $f(y) \in X^{0}$. Hence, $P_{y}$ must contain a non-preserved edge.

We claim that each edge of $Y$ is contained in at most $t-1$ of the paths $\left\{P_{y}\right\}_{y \in U \cap Y^{0}}$. Note that for $1 \leq j \leq k$, the map that takes $y \in U \cap Y^{0}$ to the $j$-th edge in the path $P_{y}$ is an injection. Thus, an edge labeled $\hat{e}_{i}(i \in[d])$ is contained in at most $\left|\alpha_{i}\right| \leq t-1$ of these paths, yielding the claim. Since every such path contains an edge which is not preserved by $f$, it follows that $U$ contains at least $\frac{\left|U \cap Y^{0}\right|}{t-1}$ non-preserved edges. Summing over all connected components $U$ yields

$$
\|f\|_{S} \geq \frac{1}{|Y|} \sum_{U} \frac{\left|U \cap Y^{0}\right|}{t-1}=\frac{1}{t^{d}-1} \cdot \frac{\left|Y^{0}\right|}{t-1}=\frac{t^{d}-1-d(t-1)}{\left(t^{d}-1\right) \cdot(t-1)} \geq \Omega_{t \rightarrow \infty}\left(\frac{1}{t}\right) .
$$

\section{Discussion}

In this work we proved that finitely generated abelian groups are polynomially stable, and bounded their degree of polynomial stability. For the free abelian group $\mathbb{Z}^{d}$, our lower and upper bounds on the degree are, respectively, $d$ and an exponential expression in $d$. It would be interesting to close this gap. We note that the exponential term in our upper bound comes from the $2^{d}$-factor in the right-hand side of Equation (4.27). More precisely, replacing this factor in Equation (4.27) by some smaller term, would yield the same replacement in Equation (4.3).

In particular, when $d$ is small, some of our lemmas can be simplified. For instance, in a lattice of degree $\leq 4$, the vectors yielding the local minima form a basis (see p. 51 of [16], cf. Proposition 4.3). This fact may be helpful in computing the exact degree of polynomial stability for certain constant values of $d$, starting with $d=2$. 
Another open question that suggests itself is whether polynomial stability holds for a larger class of groups, for example, groups of polynomial growth.

One may also consider a more flexible notion of stability (see Section 4 of [2]): In our definition of the global defect of an $\mathbb{F}_{m}$-set $X$, we do not allow adding points to $X$. It is also natural to consider a model where adding points is allowed. More precisely, given two finite $\mathbb{F}_{m}$-sets $X$ and $Y,|X| \leq|Y|$, we allow making $X$ isomorphic to $Y$ by first adding $|Y|-|X|$ points, and then adding and modifying edges. We set the cost of edge addition and edge modification to $\frac{1}{|X|}$ per edge. This generalizes Definition 3.2.

Note that our proof of Theorem 1.17 does not hold under the above model, since one can transform $X_{t}$ to a $\mathbb{Z}^{d}$-set by augmenting it with a single point, and then changing a constant number of edges. We do have a proof (not included in the present paper) applicable for this model, that the degree of polynomial stability of $\mathbb{Z}^{d}(d \geq 2)$ is at least 2 . We do not know of a better bound.

In regard to the applications to property testing (Section 1.3), our observation that an equation-set is polynomially stable if and only if its canonical tester is efficient, raises the question of which sets of equations admit any efficient tester. The subject of [3] is a similar question in the non-quantitative setting.

Finally, we note that the proof of Proposition 4.30 gives, in fact, a stronger statement: Each orbit of the constructed set $Y$ is of size at most $O\left(\frac{1}{\delta}\right)$. So, for every finite $\mathbb{F}_{m}$-set $X$ and $\delta>\frac{\left|X \backslash X_{E}\right|}{|X|}$, the set $X$ is $O\left(\delta^{1 / Q}\right)$-close to a $\Gamma$-set $Y$ whose orbits are of size at most $O\left(\frac{1}{\delta}\right)$.

\section{Acknowledgments}

We, the authors, would like express our gratitude to Alex Lubotzky for his patient and devoted support, for his encouragement, and for many important suggestions regarding the presentation of the ideas in this work. We are also indebted to Nati Linial for his support and help.

\section{A Stability of certain quotients and finite groups}

The first goal of this appendix is to prove Proposition A.3, which relates the stability rate of a group $\Gamma$ and some of its quotients. This proposition is of independent interest, and also enables a somewhat simplified approach to the proof of our main theorem (Theorem 1.16), as described in the beginning of Section 4.

Lemma A.1. Let $\mathbb{F}$ be a free group on a finite set $S$, and $E \subseteq \mathbb{F}$ a finite subset. Let $X$ and $Y$ be finite $\mathbb{F}$-sets, $|X|=|Y|$. Then,

$$
L_{E}(Y) \leq L_{E}(X)+\left(\sum_{w \in E}|w|\right) \cdot d_{S}(X, Y) .
$$




\section{ABELIAN GROUPS ARE POLYNOMIALLY STABLE}

Consequently,

$$
L_{E}(Y) \leq\left(\sum_{w \in E}|w|\right) \cdot G_{E}(Y) .
$$

Proof. Write $n=|X|=|Y|$. Recalling the notation of Section 3.1, let $\Phi_{X}, \Phi_{Y}$ : $S \rightarrow \operatorname{Sym}(n)$ be $S$-assignments such that $\mathbb{F}\left(\Phi_{X}\right)$ is isomorphic to $X, \mathbb{F}\left(\Phi_{Y}\right)$ is isomorphic to $Y$, and $d_{S}(X, Y)=d_{n}\left(\Phi_{X}, \Phi_{Y}\right)$. Then, using the triangle inequality and Lemma 2.1, we see that

$$
\begin{aligned}
L_{E}(Y) & =L_{E}\left(\Phi_{Y}\right) \\
& =\sum_{w \in E} d_{n}\left(\Phi_{Y}(w), 1\right) \\
& \leq \sum_{w \in E} d_{n}\left(\Phi_{Y}(w), \Phi_{X}(w)\right)+\sum_{w \in E} d_{n}\left(\Phi_{X}(w), 1\right) \\
& \leq \sum_{w \in E}|w| \cdot d_{n}\left(\Phi_{Y}, \Phi_{X}\right)+L_{E}\left(\Phi_{X}\right) \\
& =\left(\sum_{w \in E}|w|\right) \cdot d_{S}(X, Y)+L_{E}(X)
\end{aligned}
$$

We turn to the last assertion (A.2). Assume, for the sake of contradiction, that $L_{E}(Y)>\left(\sum_{w \in E}|w|\right) \cdot G_{E}(Y)$. Write $\Gamma=\mathbb{F} /\langle\langle E\rangle\rangle$. Then, there is a $\Gamma$-set $Z$ such that $L_{E}(Y)>\left(\sum_{w \in E}|w|\right) \cdot d_{S}(Z, Y)$. But $L_{E}(Z)=0$, and so, applying Inequality (A.1) with $X=Z$, we get a contradiction.

Definition A.2. Let $F_{1}, F_{2}:(0,|E|] \rightarrow[0, \infty)$ be monotone nondecreasing functions. Write $F_{1} \precsim F_{2}$ if $F_{1}(\delta) \leq F_{2}(C \delta)+C \delta$ for some $C>0$. Recalling the equivalence relation $\sim$ from Definition 1.10, we have $F_{1} \sim F_{2}$ if and only if $F_{1} \precsim F_{2}$ and $F_{2} \precsim F_{1}$. The partial order $\precsim$ on functions enables us to define a partial order on $\sim$-equivalence classes, namely, $\left[F_{1}\right] \precsim\left[F_{2}\right]$ if and only if $F_{1} \precsim F_{2}$.

The following proposition relates the stability rate (see Definition 1.12) of a group $\Gamma$ to the stability rate of certain quotients $\Gamma / N$.

Proposition A.3. Let $\Gamma$ be a finitely-presented group. Let $N$ be a normal subgroup of $\Gamma$. Assume that $N$ is a finitely-generated group. Then, $\mathrm{SR}_{\Gamma / N} \precsim$ $C \cdot \mathrm{SR}_{\Gamma}$ for some $C=C(\Gamma, N)>0$. In particular: (i) if $\Gamma$ is stable, then so is $\Gamma / N$, and (ii) $\operatorname{deg}\left(\mathrm{SR}_{\Gamma / N}\right) \leq \operatorname{deg}\left(\mathrm{SR}_{\Gamma}\right)$.

Proof. Let $\pi: \mathbb{F} \rightarrow \Gamma$ be a presentation of $\Gamma$ as a quotient of a finitely-generated free group $\mathbb{F}=\mathbb{F}_{S},|S|<\infty$. Then, $\pi^{-1}(N)$ is a normal subgroup of $\mathbb{F}$, $\pi^{-1}(N) / \operatorname{Ker} \pi \cong N$ is finitely-generated and $\mathbb{F} / \pi^{-1}(N) \cong \Gamma / N$. Let $E_{1} \subseteq$ $\operatorname{Ker} \pi$ be a finite set which generates $\operatorname{Ker} \pi$ as a normal subgroup of $\mathbb{F}$. Let $E_{2} \subseteq \pi^{-1}(N)$ be a finite set whose image in $\pi^{-1}(N) / \operatorname{Ker} \pi$ generates the group $\pi^{-1}(N) / \operatorname{Ker} \pi$. Then, $E=E_{1} \cup E_{2}$ generates $\pi^{-1}(N)$ as a normal subgroup of $\mathbb{F}$. So, $\mathbb{F} /\left\langle\left\langle E_{1}\right\rangle\right\rangle \cong \Gamma$ and $\mathbb{F} /\langle\langle E\rangle \rrbracket \cong \Gamma / N$. Thus, it suffices to show that $\operatorname{SR}_{E}(\delta) \leq O\left(\operatorname{SR}_{E_{1}}(\delta)+\delta\right)$. 
Let $X$ be a finite $\mathbb{F}$-set. Write $n=|X|$ and $\delta=L_{E}(X)$. We need to show that $G_{E}(X) \leq O\left(\operatorname{SR}_{E_{1}}(\delta)+\delta\right)$. Let $Z$ be a $\Gamma$-set, $|Z|=|X|=n$, for which $d_{S}(X, Z) \leq \operatorname{SR}_{E_{1}}\left(L_{E_{1}}(X)\right)$. Since $E_{1} \subseteq E$, we have $L_{E_{1}}(X) \leq L_{E}(X)$, and so

$$
d_{S}(X, Z) \leq \operatorname{SR}_{E_{1}}\left(L_{E}(X)\right)=\operatorname{SR}_{E_{1}}(\delta) .
$$

Using Lemma A.1, we deduce

$$
\begin{aligned}
L_{E_{2}}(Z) \leq L_{E}(Z) & \leq L_{E}(X)+\left(\sum_{w \in E}|w|\right) \cdot d_{S}(X, Z) \\
& \leq \delta+\left(\sum_{w \in E}|w|\right) \cdot \operatorname{SR}_{E_{1}}(\delta)
\end{aligned}
$$

This allows use to bound the size of $Z_{E_{2}}$ (see Definition 3.6) from below. Indeed, there are $n \cdot L_{E_{2}}(Z)$ pairs $(z, w) \in Z \times E$ for which $w \cdot z \neq z$. Clearly, the number of distinct values of $z$ in those pairs is at most $n \cdot L_{E_{2}}(Z)$, and so

$$
\left|Z_{E_{2}}\right| \geq n-n \cdot L_{E_{2}}(Z) \geq n-n \cdot\left(\delta+\left(\sum_{w \in E}|w|\right) \cdot \operatorname{SR}_{E_{1}}(\delta)\right) .
$$

Now, as $N=\left\langle\pi\left(E_{2}\right)\right\rangle$, each $z \in Z_{E_{2}}$ is fixed by $N$. In fact, since $N$ is normal in $\Gamma$, every $z \in Z_{E_{2}}$ belongs to an orbit $\Gamma \cdot z \subseteq Z$ where all points are fixed by $N$. Therefore, $Z_{E_{2}}$ is a union of $\Gamma$-orbits of $Z$, and the action of $\Gamma$ on $Z_{E_{2}}$ factors through $\Gamma / N$. Consider the inclusion map $\iota: Z_{E_{2}} \rightarrow Z$. All points of $Z_{E_{2}}$ are equivariance points of $\iota$. So, applying Lemma 3.15 to $\iota$, we get

$$
\begin{aligned}
G_{E}(Z) & \leq|S| \cdot\left(1-\frac{1}{n} \cdot\left|Z_{E_{2}}\right|\right) \\
& \leq|S| \cdot\left(\delta+\left(\sum_{w \in E}|w|\right) \cdot \operatorname{SR}_{E_{1}}(\delta)\right) \\
& \leq O\left(\operatorname{SR}_{E_{1}}(\delta)+\delta\right) .
\end{aligned}
$$

Hence,

$$
\begin{aligned}
G_{E}(X) & \leq G_{E}(Z)+d_{S}(X, Z) \\
& \leq O\left(\operatorname{SR}_{E_{1}}(\delta)+\delta\right)+\operatorname{SR}_{E_{1}}(\delta) \\
& \leq O\left(\operatorname{SR}_{E_{1}}(\delta)+\delta\right),
\end{aligned}
$$

as required.

Theorem 2 of [9] states that all finite groups are stable. The following is the quantitative version:

Proposition A.4. Let $\Gamma$ be a finite group. Then, the degree of polynomial stability $\operatorname{deg}\left(\mathrm{SR}_{\Gamma}\right)$ of $\Gamma$ is 1 . In particular, $\Gamma$ is stable. 


\section{ABELIAN GROUPS ARE POLYNOMIALLY STABLE}

Proof. Write $\delta \mapsto \delta$ for the inclusion map $(0,|E|] \rightarrow(0, \infty]$, and $[\delta \mapsto \delta]$ for its $\sim$-class (Definition 1.10). Our task is to show that $\mathrm{SR}_{\Gamma}=[\delta \mapsto \delta]$. By the definition of the relation $\precsim$, we have $[\delta \mapsto \delta] \precsim \mathrm{SR}_{\Gamma}$, and we need to prove the reverse inequality. Let $\pi: \mathbb{F} \rightarrow \Gamma$ be a presentation of $\Gamma$ as a quotient of a finitely-generated free group $\mathbb{F}$. Then, $[\mathbb{F}: \operatorname{Ker} \pi]<\infty$, and so $\operatorname{Ker} \pi$ itself is a finitely-generated group. Therefore, by Proposition A.3, for some $C>0$,

$$
\mathrm{SR}_{\Gamma} \precsim C \cdot \mathrm{SR}_{\mathbb{F}} \sim[\delta \mapsto \delta] .
$$

The following proposition shows that for every equation-set, except for the trivial equation-sets $\emptyset$ and $\{1\}$, the stability rate is at least linear. This motivates the requirement $k \geq 1$ in Definition 1.13.

Proposition A.5. Let $\mathbb{F}$ be a free group on a finite set $S$, and $E \subseteq \mathbb{F}$ a finite subset. Assume that $E \neq \emptyset$ and $E \neq\left\{1_{\mathbb{F}}\right\}$. Then,

$$
\mathrm{SR}_{E}(\delta) \geq C \cdot \delta
$$

for some $C>0$.

Proof. Since $\mathrm{SR}_{E}$ is monotone non-decreasing, it suffices to prove Inequality (A.3) for $\delta \in\left(0, \delta_{0}\right] \cap \mathbb{Q}$ for some $\delta_{0}>0$. As $E$ contains a non-trivial element of $\mathbb{F}$, there is a finite $\mathbb{F}$-set $X$ such that $\delta_{0} \stackrel{\text { def }}{=} L_{E}(X)$ is positive (and rational). Write $\delta_{0}=\frac{m_{0}}{n_{0}}$ for integers $0<m_{0} \leq n_{0}$. Take $\delta \in\left(0, \delta_{0}\right] \cap \mathbb{Q}$, and write $\delta=\frac{p}{q} \cdot \delta_{0}$, where $0<p \leq q$ are integers. Define an $\mathbb{F}$-set $Y$ as the disjoint union of $p$ copies of $X$ and $(q-p) \cdot|X|$ additional fixed points. Then,

$$
L_{E}(Y)=\frac{p \cdot|X| \cdot L_{E}(X)}{p \cdot|X|+(q-p) \cdot|X|}=\frac{p}{q} \cdot L_{E}(X)=\delta .
$$

Finally, by Inequality (A.2) in Lemma A.1, $G_{E}(Y) \geq\left(\sum_{w \in E}|w|\right)^{-1} \cdot L_{E}(Y)$, and so $\operatorname{SR}_{E}(\delta) \geq\left(\sum_{w \in E}|w|\right)^{-1} \cdot \delta$, as required.

\section{References}

[1] Goulnara Arzhantseva and Liviu Păunescu, Almost commuting permutations are near commuting permutations, J. Funct. Anal. 269 (2015), no. 3, 745-757.

[2] Oren Becker and Alexander Lubotzky, Group stability and Property (T), available at https://arxiv.org/abs/1809.00632.

[3] _ Testability of permutation equations and group stability (In preparation).

[4] Oren Becker, Alexander Lubotzky, and Andreas Thom, Stability and Invariant Random Subgroups, available at https://arxiv.org/abs/1801.08381.

[5] Marcus De Chiffre, Lev Glebsky, Alexander Lubotzky, and Andreas Thom, Stability, cohomology vanishing, and non-approximable groups, available at https://arxiv.org/abs/1711.10238. 


\section{OREN BECKER AND JONATHAN MOSHEIFF}

[6] S. Eilers, T. Shulman, and A. P. W. Sørensen, $C^{*}$-stability of discrete groups, available at https://arxiv.org/abs/1808.06793.

[7] Gábor Elek and Endre Szabó, Sofic representations of amenable groups, Proc. Amer. Math. Soc. 139 (2011), no. 12, 4285-4291.

[8] Nikolay Filonov and Ilya Kachkovskiy, A Hilbert-Schmidt analog of Huaxin Lin's Theorem, available at https://arxiv.org/abs/1008.4002.

[9] Lev Glebsky and Luis Manuel Rivera, Almost solutions of equations in permutations, Taiwanese J. Math. 13 (2009), no. 2A, 493-500.

[10] Oded Goldreich, Introduction to property testing, Cambridge University Press, 2017.

[11] Don Hadwin and Tatiana Shulman, Stability of group relations under small HilbertSchmidt perturbations, J. Funct. Anal. 275 (2018), no. 4, 761-792.

[12] - Tracial stability for $C^{*}$-algebras, Integral Equations Operator Theory 90 (2018), no. 1 , Art. 1,35

[13] M. B. Hastings, Making almost commuting matrices commute, Comm. Math. Phys. 291 (2009), no. 2, 321-345.

[14] J. C. Lagarias, H. W. Lenstra Jr., and C.-P. Schnorr, Korkin-Zolotarev bases and successive minima of a lattice and its reciprocal lattice, Combinatorica 10 (1990), no. 4, $333-348$.

[15] Jeffrey C. Lagarias, Point lattices, Handbook of combinatorics, Vol. 1, 2, 1995, pp. 919966.

[16] Jacques Martinet, Perfect lattices in Euclidean spaces, Grundlehren der Mathematischen Wissenschaften [Fundamental Principles of Mathematical Sciences], vol. 327, SpringerVerlag, Berlin, 2003.

[17] Donald S. Ornstein and Benjamin Weiss, Entropy and isomorphism theorems for actions of amenable groups, J. Analyse Math. 48 (1987), 1-141.

[18] Dana Ron, Algorithmic and analysis techniques in property testing, Found. Trends Theor. Comput. Sci. 5 (2009), no. 2, front matter, 73-205.

[19] S. M. Ulam, A collection of mathematical problems, Interscience Tracts in Pure and Applied Mathematics, no. 8, Interscience Publishers, New York-London, 1960. 